\title{
Supersymmetric spindles
}

\author{
Pietro Ferrero, ${ }^{a}$ Jerome P. Gauntlett ${ }^{b}$ and James Sparks ${ }^{a}$ \\ ${ }^{a}$ Mathematical Institute, University of Oxford, \\ Andrew Wiles Building, Radcliffe Observatory Quarter, \\ Woodstock Road, Oxford, OX2 6GG, U.K. \\ ${ }^{b}$ Blackett Laboratory, Imperial College, \\ Prince Consort Rd., London, SW7 2AZ, U.K. \\ E-mail: pietro.ferrero@gtc.ox.ac.uk, j.gauntlett@imperial.ac.uk, \\ sparks@maths.ox.ac.uk
}

ABSTRACT: In the context of holography, we analyse aspects of supersymmetric geometries based on two-dimensional orbifolds known as spindles. By analysing $\operatorname{spin}^{c}$ spinors on a spindle with an azimuthal rotation symmetry we show that under rather general conditions there are just two possibilities, called the 'twist' and the 'anti-twist', which are determined by the quantized magnetic flux through the spindle. A special case of the twist is the standard topological twist which is associated with constant and chiral spinors. We construct solutions of $D=5$ and $D=4$ STU gauged supergravity theories that are dual to D3branes and M2-branes wrapping spindles, respectively, which realize both the anti-twist, as seen before, but also the twist. For the wrapped D3-brane solutions we reproduce the central charge of the gravity solution from the dual field theory by analysing the anomaly polynomial of $\mathcal{N}=4 \mathrm{SYM}$ theory. We also discuss M5-branes wrapped on spindles both from a gravity and a field theory point of view.

Keywords: AdS-CFT Correspondence, Anomalies in Field and String Theories, Gaugegravity correspondence, M-Theory

ArXiv EPrint: 2112.01543 


\section{Contents}

1 Introduction $\quad 2$

2 Twists and anti-twists 4

2.1 Set-up 4

2.2 Orbibundles on spindles 6

2.3 Spinors on spindles 9

$\begin{array}{ll}2.4 \text { Topological twist } & 15\end{array}$

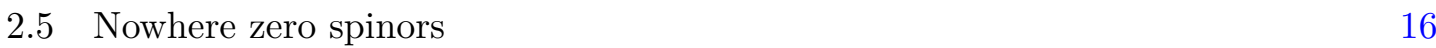

$\begin{array}{ll}2.6 & \text { Uplifted solutions } \\ \end{array}$

3 D3-branes 18

$\begin{array}{ll}3.1 & \text { The } D=5 \text { gauged supergravity model } \\ \end{array}$

$\begin{array}{lll}3.2 & \text { Supergravity solution and global analysis } & 20\end{array}$

3.3 Killing spinors 24

3.4 Comparison with field theory 27

$\begin{array}{ll}3.5 & \text { M5-branes }\end{array}$

4 M2-branes $\quad 30$

4.1 The $D=4$ gauged supergravity model $\quad 30$

$\begin{array}{lll}4.2 & \text { Supergravity solution and global analysis } & 31\end{array}$

4.3 Killing spinors 34

5 M5-branes $\quad 36$

5.1 The $D=7$ gauged supergravity model 36

$\begin{array}{lll}5.2 & \text { Supergravity solution and Killing spinors } & 37\end{array}$

5.3 Comparison with field theory 40

6 Final comments $\quad 41$

A Spheres from spindles $\quad 43$

A.1 $\mathrm{AdS}_{3} \times S^{2}$ solutions as a limit 43

A.2 $\mathrm{AdS}_{2} \times S^{2}$ solutions as a limit $\quad 45$

A.3 $\operatorname{AdS}_{5} \times S^{2}$ solutions as a limit 46

$\begin{array}{ll}\text { B Killing spinors in AdS } & 48\end{array}$

C Killing spinors for the D3-brane solutions 49 


\section{Introduction}

A new facet of the AdS/CFT correspondence involves a class of two-dimensional orbifolds known as spindles [1]. Recall that a spindle is topologically a two-sphere but with quantized conical deficit angles at the two poles. More precisely, we define a spindle $\Sigma=\mathbb{W} \mathbb{C P}_{\left[n_{1}, n_{2}\right]}^{1}$ to be a weighted projective space that is specified by two positive, co-prime integers $n_{1}, n_{2}$. Various $\operatorname{AdS} \times \Sigma$ supersymmetric solutions of gauged supergravity theories in $D=4,5$ and 7 dimensions have now been constructed, which are naturally associated with ${ }^{1} \mathrm{M} 2$, D3 and M5-branes, respectively [1,3-8]. These can then be uplifted on certain compact manifolds to give supersymmetric solutions of $D=10$ and $D=11$ supergravity that are dual to new SCFTs. Remarkably, in the examples involving M2 and D3-branes, one finds uplifted solutions that are completely regular and free from any orbifold singularities. In other examples, involving M5-branes, orbifold singularities remain in the $D=11$ solutions, but strong supporting evidence has been provided that they are in fact dual to bona fide SCFTs.

The regular uplifted solutions in $D=10$ and $D=11$ supergravity just mentioned were in fact first constructed some time ago using a different approach $[9,10]$, but the dual SCFTs remained obscure. The new insight that they can be recast as uplifted solutions of a lower-dimensional gauged supergravity leads to the natural conjecture that the dual SCFTs are the low-energy limit of the field theories arising from branes wrapping a spindle. Evidence supporting this picture has been provided in the examples involving D3 and M5branes, by calculating the central charge of the proposed SCFT using anomaly polynomials, and finding exact agreement with the gravity result.

The picture that has emerged has a direct parallel with the well-known constructions involving branes wrapping Riemann surfaces $\Sigma_{g}$, with genus $g$, but there are some crucial differences. In the standard constructions, starting with [11], supersymmetry on the worldvolume of the branes is realized with a partial "topological twist", which involves switching on background R-symmetry gauge fields that are determined, locally, by the spin connection of the metric on the Riemann surface. An immediate consequence of the topological twist is that the integrated R-symmetry flux through the Riemann surface, suitably normalized, is equal to the Euler number of the Riemann surface. Another consequence of the topological twist is that the Killing spinors for the $\operatorname{AdS} \times \Sigma_{g}$ solutions are chiral and constant on $\Sigma_{g}$. As is also well-known, this standard set-up is realized by wrapping branes on a Riemann surface that is holomorphically embedded in a Calabi-Yau manifold.

By contrast, in the new $\operatorname{AdS} \times \Sigma$ solutions involving spindles the Killing spinors are neither chiral nor constant on $\Sigma$. In fact two distinct ways of realizing supersymmetry for spindles have now emerged, which we refer to as the twist and anti-twist, and they can be characterized via the R-symmetry flux through the spindle:

$$
\begin{aligned}
\text { Twist: } & \frac{1}{2 \pi} \int_{\Sigma} F=\chi \equiv \frac{n_{1}+n_{2}}{n_{1} n_{2}}, \\
\text { Anti-twist: } & \frac{1}{2 \pi} \int_{\Sigma} F=\chi_{-} \equiv \frac{n_{2}-n_{1}}{n_{1} n_{2}},
\end{aligned}
$$

\footnotetext{
${ }^{1} \mathrm{~A}$ recent interesting paper has constructed solutions of massive type IIA that are associated with D4-branes wrapping spindles [2].
} 
where $F$ is the field strength for the R-symmetry gauge field. Notice in the case of the twist $\chi$ is in fact the Euler number of the spindle, so this is a feature that is also shared with the standard topological twist.

All of the known $\operatorname{AdS} \times \Sigma$ solutions have a spindle metric that is invariant under azimuthal rotations about the poles. One of the main results of this paper is to show, assuming such a spindle metric, that the twist and the anti-twist are the only possible ways of realizing supersymmetry on the spindle. The proof follows from analysing the regularity conditions for the two-component $\operatorname{spin}^{c}$ spinors on the spindle $\Sigma$, which are charged with respect to the R-symmetry gauge field, near each of the two orbifold points. The only other assumption that goes into the proof is that the Killing spinor should be non-vanishing at these orbifold points, which holds on general grounds for supersymmetric AdS solutions, as we shall discuss. Our analysis also clarifies why the regularity arguments that have been used for supergravity solutions with spindles constructed in [1-8] do in fact give the correct results.

For the Killing spinors arising in the supersymmetric $\operatorname{AdS} \times \Sigma$ solutions, we show that a vector bilinear which can be constructed from the Killing spinor is the Killing vector which generates the azimuthal rotations on the spindle. This is true for both the twist and the anti-twist cases. This aspect of the way in which the supersymmetry algebra is being realized on the spindle can be contrasted ${ }^{2}$ with the usual topological twist for Riemann surfaces, where the corresponding vector bilinear vanishes identically. This further underscores the fact that while the twist case for the spindle and the topological twist share the feature that the R-symmetry flux is the Euler number as in (1.1), they are in fact quite distinct. That being said, if one formally sets $n_{1}=n_{2}=1$ in the central charge of the dual SCFTs for various $\mathrm{AdS} \times \Sigma$ solutions, one gets the same result that one gets for some known $\operatorname{AdS} \times S^{2}$ solutions with a topological twist.

In this paper we will also re-examine the known constructions of $\operatorname{AdS} \times \Sigma$ solutions of various gauged supergravity theories, and find some new solutions that were missed in the original analyses. In particular, previous constructions of $\mathrm{AdS}_{3} \times \Sigma$ D3-brane solutions and $\mathrm{AdS}_{2} \times \Sigma \mathrm{M} 2$-brane solutions only realized the anti-twist in (1.1), but we shall see that for both D3-branes and M2-branes there also exist new twist solutions. ${ }^{3}$ Moreover, these twist solutions have a natural physical interpretation as the IR limit of D3-branes and M2-branes wrapped on a spindle that is holomorphically embedded inside a Calabi-Yau four-fold and five-fold, respectively. Interestingly, for $\mathrm{AdS}_{5} \times \Sigma \mathrm{M} 5$-brane solutions the construction in [6] only leads to twist solutions, while for $\mathrm{AdS}_{3} \times \Sigma \times \Sigma_{g}$ M5-brane solutions we find new twist solutions, as well as the previously known anti-twist solutions. It is an interesting open problem to identify a UV geometry for the many anti-twist solutions that have now been constructed, involving wrapped branes, a point we discuss further in section 6 .

The outline of the paper is as follows. In section 2 we give a general proof that (1.1) are the only possibilities for the R-symmetry flux through a spindle, under some rather

\footnotetext{
${ }^{2}$ From the dual field theory point of view, a closely related point is that in considering a SCFT on the spindle, one finds that the R-symmetry of the resulting lower-dimensional SCFT is a mixture of the spindle symmetry and the R-symmetry of the higher-dimensional SCFT.

${ }^{3}$ With the caveat that these require multiple magnetic charges, and thus in particular do not exist in minimal gauged supergravity, as shown in $[1,3]$.
} 
general assumptions. Our discussion includes a global description of $\mathrm{U}(1)$ orbibundles, the associated Killing spinors and some properties of the uplifted solutions. We will explain how the standard topological twist arises as a special case of the twist, but we emphasize that in actual solutions the Killing spinors have distinct features. In section 3 we present a fairly comprehensive analysis of $\mathrm{AdS}_{3} \times \Sigma$ D3-brane solutions, in particular constructing new twist solutions. We interpret the new twist solutions as near-horizon limits of D3-branes wrapped on $\Sigma$ embedded holomorphically inside a Calabi-Yau four-fold, and reproduce the supergravity central charge by computing the anomaly polynomial of $\mathcal{N}=4$ SYM theory wrapped on $\Sigma$. Some of the new $D=5$ gauged supergravity solutions also uplift to new $\mathrm{AdS}_{3} \times \Sigma \times \Sigma_{g}$ twist M5-brane solutions, or more generally the product of $\Sigma$ with a punctured Riemann surface. Section 4 presents a similar analysis for $\operatorname{AdS}_{2} \times \Sigma$ M2-brane solutions, although here the regularity conditions are more difficult to solve in closed form, and we content ourselves with showing that new twist solutions exist, without classifying them. In section 5 we briefly discuss $\mathrm{AdS}_{5} \times \Sigma$ M5-brane solutions, in particular constructing the explicit Killing spinors for the solutions found in [6]. We conclude with some discussion in section 6 . Three appendices contain some additional results. Appendix A shows how various previously known $\mathrm{AdS} \times S^{2}$ solutions arise from limits of the (local) $\operatorname{AdS} \times \Sigma$ solutions presented here, while appendix B discusses Killing spinors in AdS. In appendix $\mathrm{C}$ we present some details on our derivation of the explicit Killing spinors for the case of the D3-brane solutions; the derivation for the other cases is somewhat similar.

\section{Twists and anti-twists}

As already mentioned, several different classes of supersymmetric supergravity solutions involving spindles have recently been constructed, including [1,3-8]. Typically these are realized as solutions to a lower-dimensional gauged supergravity theory, and then uplifted to $D=10$ string theory or $D=11$ M-theory solutions, where the solutions describe D3-brane, M2-brane or M5-brane backgrounds. In every case the total magnetic flux for the R-symmetry gauge field through the spindle $\Sigma$ has been found to be one of the two possibilities in (1.1). Here $A$ is the Abelian R-symmetry gauge field, with curvature $F=\mathrm{d} A$, normalized so that the Killing spinor has charge $\frac{1}{2}$.

In this section we give a general proof that these are the only two possibilities, under some fairly general assumptions: (i) azimuthal rotations of the spindle are a symmetry of the solution, (ii) the Killing spinor is non-zero at the poles of the spindle. We shall discuss the assumption (ii) further in section 2.5, where we argue that the Killing spinor is in fact nowhere zero for supersymmetric AdS solutions in string and M-theory. The precise definition of assumption (i) will be made clear in the next subsections. The proof follows from global regularity conditions on the Killing spinor, independently of precisely which equation it solves.

\section{$2.1 \quad$ Set-up}

We begin in this subsection by summarizing the basic set-up. We focus on a gauged supergravity perspective, as this is the framework where we construct solutions in later 
sections, but we also discuss the implications for the uplifted supergravity solutions in section 2.6.

The precise details of the Killing spinor equation will not be relevant for what follows. In general we can take the Killing spinor to carry a possible "flavour index" and write $\zeta^{i}$, which for each $i=1, \ldots, n$ is a two-component $\operatorname{spin}^{c}$ spinor on $\Sigma$. We assume it satisfies ${ }^{4}$ a Killing spinor equation (KSE) of the schematic form

$$
\left(\nabla-\frac{\mathrm{i}}{2} A\right) \zeta^{i}+\mathcal{M}_{j}^{i} \zeta^{j}=0 .
$$

Here $\nabla=\mathrm{d}+\frac{1}{4} \omega^{a b} \gamma_{a b}$ is the Levi-Civita spin connection and, for definiteness, we have taken the spinor to have charge $\frac{1}{2}$ with respect to the R-symmetry gauge field $A$. The matrix $\mathcal{M}^{i}{ }_{j}$ could depend on scalar fields, as well as other gauge fields that act on the flavour indices but, as we will see, this matrix will not play any role in the following discussion; indeed we can suppress the flavour index on $\zeta^{i}$ and simply write $\zeta$.

We will be interested in studying such Killing spinors $\zeta$ on a spindle. By definition, a spindle $\Sigma$ is topologically a two-sphere with conical deficit angles $2 \pi\left(1-1 / n_{S}\right)$ at the "south pole" and $2 \pi\left(1-1 / n_{N}\right)$ at the "north pole", where $n_{S}, n_{N} \in \mathbb{N}$ are relatively prime with $\operatorname{hcf}\left(n_{S}, n_{N}\right)=1$. This may equivalently be described as the weighted projective space $\Sigma=\mathbb{W} \mathbb{C P}_{\left[n_{S}, n_{N}\right]}^{1}$, which is an orbifold Riemann surface. We will exclude the special case $n_{S}=n_{N}=1$ from the definition of a spindle, but point out that these values give rise to a smooth two-sphere. We assume that the metric on the spindle is invariant under azimuthal rotations around the poles. That is, there is a Killing vector $k \equiv \partial_{\varphi}$, with coordinates $(\rho, \varphi)$ in which the metric on the spindle takes the form

$$
\mathrm{d} s_{\Sigma}^{2}=\mathrm{d} \rho^{2}+f^{2}(\rho) \mathrm{d} \varphi^{2} .
$$

We normalize $\varphi$ to have period $\Delta \varphi=2 \pi$, and take $\rho \in\left[\rho_{1}, \rho_{2}\right]$. The metric (2.2) describes a spindle with the above deficit angles if $f(\rho)$ has the boundary behaviour $f(\rho)=(\rho-$ $\left.\rho_{1}\right) / n_{N}+O\left(\left(\rho-\rho_{1}\right)^{2}\right)$ and $f(\rho)=\left(\rho_{2}-\rho\right) / n_{S}+O\left(\left(\rho_{2}-\rho\right)^{2}\right)$ near the north and south poles, respectively, with $f$ strictly positive on the open interval $\left(\rho_{1}, \rho_{2}\right)$.

The Killing vector $k=\partial_{\varphi}$ is assumed to generate a symmetry of the full solution. In particular we assume that the matrix $\mathcal{M}$ in the $\operatorname{KSE}(2.1)$ invariant, i.e. $\mathcal{L}_{k} \mathcal{M}=0$, where $\mathcal{L}_{k}$ is the Lie derivative. Similarly we assume that $\mathcal{L}_{k} F=0$, and will typically work in local gauges which satisfy $\mathcal{L}_{k} A=0$, and hence we can write

$$
A=a(\rho) \mathrm{d} \varphi .
$$

Notice this leaves a freedom to make local gauge transformations

$$
A \rightarrow A+\gamma \mathrm{d} \varphi
$$

with $\gamma$ a constant, which equivalently shifts $a(\rho) \rightarrow a(\rho)+\gamma$. We shall present a careful global discussion of gauge fields on spindles in section 2.2 below.

\footnotetext{
${ }^{4}$ The Killing spinor might also satisfy additional algebraic equations, but these play no role in the following arguments.
} 
With the above assumptions, the $\mathrm{KSE}$ (2.1) is invariant under $k$. A consequence is that the Killing spinor solution $\zeta$ has definite charge $Q$ under $k=\partial_{\varphi}$. That is, $\mathcal{L}_{k} \zeta=\mathrm{i} Q \zeta$, where $\mathcal{L}_{k}=\nabla_{k}+\frac{1}{8}\left(\mathrm{~d} k^{b}\right)^{a b} \gamma_{a b}$ denotes the spinor Lie derivative along $k$, where $k^{b}$ is the one-form metrically dual to $k$. Indeed, since the KSE is invariant under $k$, its space of solutions will form a representation of the $\mathrm{U}(1)$ symmetry generated by $k$. But the irreducible representations are then one-dimensional, classified by their charge $Q$, and it follows that without loss of generality we may take a solution $\zeta$ with definite charge. ${ }^{5}$ Notice that the charge $Q$ of the Killing spinor depends on the residual local gauge freedom (2.4), which acts on $\zeta$ as

$$
\zeta \rightarrow \mathrm{e}^{\mathrm{i} \gamma \varphi / 2} \zeta
$$

and thus shifts $Q \rightarrow Q+\gamma / 2$.

\subsection{Orbibundles on spindles}

Since a spindle is a singular space, with orbifold singularities at its poles, we need to be careful about what we mean by a "regular gauge field" and "regular spinor" on a spindle. We begin in this subsection with a global description of an Abelian gauge field on an orbifold Riemann surface, in terms of a connection on a principal U(1)-orbibundle, and then move on to spinors in section 2.3.

In appendix $\mathrm{A}$ of [3], $\mathrm{U}(1)$-orbibundles over spindles were described in terms of the quotient of $S^{3}$ by a weighted U(1) action on $\mathbb{C}^{2} \supset S^{3}$. This makes use of the fact that a locally free ${ }^{6}$ action of a compact Lie group $G$ on a manifold $M$ has orbit space $M / G$ which is naturally an orbifold, with the total space $M$ then being a principal $G$-orbibundle over $M / G$. Starting with $M=S^{3}$ gives a particular $\mathrm{U}(1)$-orbibundle over the spindle $\Sigma=S^{3} / \mathrm{U}(1)$, and different bundles may then be constructed by factoring through the Lens space quotient $S^{3} / \mathbb{Z}_{\lambda}$, where $\mathbb{Z}_{\lambda} \subset \mathrm{U}(1)$. Here, we instead present the more usual local description of orbibundles, and associated connections, in terms of covering spaces and gluing, specializing to the case of interest where $G=\mathrm{U}(1)$ is fibred over a spindle.

We begin with a local model near to one of the poles of the spindle (or a more general orbifold Riemann surface). Thus let $\mathbb{C} \times S^{1}$ be a trivial principal $\mathrm{U}(1)=S^{1}$-bundle over $\mathbb{C} \cong \mathbb{R}^{2}$, and introduce complex coordinates $(z, \xi)$, where $|\xi|=1$ is a unit norm complex number parametrizing the circle fibre. The copy of $\mathbb{C}$ will be the covering space for $U \equiv \mathbb{C} / \mathbb{Z}_{n}$, where the generator of $\mathbb{Z}_{n}$ acts on $z \in \mathbb{C}$ via $z \mapsto \omega z$, with $\omega \equiv \mathrm{e}^{2 \pi \mathrm{i} / n}$ being a primitive $n^{\text {th }}$ root of unity. In general, to specify a principal $G$-orbibundle over $\mathbb{R}^{k} / \Gamma$, one picks a homomorphism $h$ from the local orbifold group $\Gamma$ into the fibre group $G$. In the current setting this amounts to picking a "charge" $m \in \mathbb{Z}$ :

$$
h: \mathbb{Z}_{n} \rightarrow \mathrm{U}(1), \quad \text { where } h(\omega)=\omega^{m} .
$$

More precisely, since $\omega^{n}=1$, we have $m \in \mathbb{Z}_{n}$. A principal $G$-orbibundle over $\mathbb{R}^{k} / \Gamma$ is then defined as the quotient $B \equiv\left(\mathbb{R}^{k} \times G\right) / \Gamma$, where the action on $G$ is given by the homomorphism $h$. In our case this is

$$
B \equiv\left(\mathbb{C} \times S^{1}\right) / \mathbb{Z}_{n}, \quad \text { where } \omega \cdot(z, \xi)=\left(\omega z, \omega^{m} \xi\right) .
$$

\footnotetext{
${ }^{5}$ Equivalently, notice that if $\zeta$ solves the $\mathrm{KSE}$ then so does $\mathcal{L}_{k} \zeta$, which is then also a Killing spinor.

${ }^{6}$ Meaning all isotropy groups are finite.
} 
The bundle projection map $\pi: B \rightarrow U=\mathbb{C} / \mathbb{Z}_{n}$ projects onto the first factor. Since the action of $h(\Gamma)$ on the fibre $G$ is free, it follows that the total space of $B$ will be smooth (i.e. the lifted action of $\Gamma$ using $h$ has no fixed points) if $h$ is injective. In our case, this is so precisely ${ }^{7}$ when $\operatorname{hcf}(m, n)=1$.

We may further analyse this local model by writing $\xi=\mathrm{e}^{\mathrm{i} \chi}, z=\rho \mathrm{e}^{\mathrm{i} \hat{\phi}}$, where $\rho \geq 0$ is a radial coordinate, and on the covering space $\mathbb{C} \times S^{1}$ we have $\Delta \chi=\Delta \hat{\phi}=2 \pi$. We may geometrize a connection one-form for the $\mathrm{U}(1)$ bundle by writing the metric

$$
\mathrm{d} s_{\mathbb{C} \times S^{1}}^{2}=\left(\mathrm{d} \chi+A_{(0)}\right)^{2}+\mathrm{d} \rho^{2}+h(\rho)^{2} \mathrm{~d} \hat{\phi}^{2},
$$

where $h(\rho)=\rho+O\left(\rho^{2}\right)$ near $\rho=0$ in order for this rotationally-invariant metric on $\mathbb{C}$ to be smooth, and importantly $A_{(0)}$ is an arbitrary global one-form on $\mathbb{C}$ that is invariant under the $\mathbb{Z}_{n}$ action. For the case of interest in the paper we impose the stronger condition that

$$
\mathcal{L}_{\partial_{\hat{\phi}}} A_{(0)}=0,
$$

so that rotations of $\mathbb{C}$ generated by $\partial_{\hat{\phi}}$ are a symmetry. The generator of $\mathbb{Z}_{n}$ acts on the angular coordinates as

$$
(\chi, \hat{\phi}) \mapsto\left(\chi+\frac{2 \pi m}{n}, \hat{\phi}+\frac{2 \pi}{n}\right) .
$$

We may then make the $\mathrm{SL}(2, \mathbb{Z})$ transformation of the torus parametrized by $(\chi, \hat{\phi})$ given by

$$
\left(\begin{array}{l}
\psi \\
\phi
\end{array}\right) \equiv\left(\begin{array}{cc}
1 & -m \\
0 & 1
\end{array}\right)\left(\begin{array}{l}
\chi \\
\hat{\phi}
\end{array}\right),
$$

with $\Delta \psi=\Delta \phi=2 \pi$ on the covering space. Notice that this transformation is only defined for $\rho>0$, as the azimuthal coordinate $\hat{\phi}$ is not defined at the origin $\rho=0$ of $\mathbb{C}$. Moreover, although so far $m \in \mathbb{Z}_{n}$, the transformation (2.11) requires picking a particular choice of integer lift $m \in \mathbb{Z}$. This then introduces some redundancy/gauge freedom in the subsequent description, that we comment on further below. The new coordinate $\psi=\chi-m \hat{\phi}$ is invariant under (2.10), while the metric (2.8) becomes

$$
\mathrm{d} s_{\mathbb{C} \times S^{1}}^{2}=(\mathrm{d} \psi+A)^{2}+\mathrm{d} \rho^{2}+h(\rho)^{2} \mathrm{~d} \phi^{2} .
$$

Notice that now $\mathbb{Z}_{n}$ acts only on $\phi$ :

$$
(\psi, \phi) \mapsto\left(\psi, \phi+\frac{2 \pi}{n}\right),
$$

while the new connection one-form is

$$
A=A_{(0)}+m \mathrm{~d} \phi .
$$

The metric on the quotient $B=\left(\mathbb{C} \times S^{1}\right) / \mathbb{Z}_{n}$ is then similarly given by (2.12), but where $\psi$ has period $2 \pi$ and $\phi$ has period $2 \pi / n$. Because of the latter period, the connection

\footnotetext{
${ }^{7}$ If $\operatorname{hcf}(m, n) \neq 1$ we can write $m=b m^{\prime}, n=b n^{\prime}$ for some $b \in \mathbb{Z}$. Then if we carry out the action of (2.7) $n^{\prime}$ times, it takes $(z, \xi) \rightarrow\left(\omega^{n / b} z, \xi\right)$, which defines a $\mathbb{Z}_{b}$ sub-action that leaves the fibre fixed.
} 
term $m \mathrm{~d} \phi$ is a non-trivial flat connection on $(\mathbb{C} \backslash\{0\}) / \mathbb{Z}_{n}$, i.e. it cannot be removed by any non-singular gauge transformation. To match with the conventions in $(2.2)$, on the quotient space we write $\phi=\varphi / n$, with $\varphi$ having period $2 \pi$, so that $A=A_{(0)}+\frac{m}{n} \mathrm{~d} \varphi$. This gauge field is not defined at the origin in $\mathbb{C}$, precisely because the azimuthal coordinates $\hat{\phi}, \varphi$ are not defined there.

The above construction shows that we may equivalently view $\mathrm{U}(1)$-orbibundles over $U=\mathbb{C} / \mathbb{Z}_{n}$ as specifying a flat connection that is pulled back from the unit circle in $(\mathbb{C} \backslash\{0\}) / \mathbb{Z}_{n}$, with holonomy precisely $\exp \left(\mathrm{i} \int_{S_{\varphi}^{1}} A_{\text {flat }}\right)=\omega^{m}=h(\omega) \in \mathrm{U}(1)$. Also notice that if $m=p n$ is an integer multiple $p$ of $n$, then $A=A_{(0)}-\mathrm{i} g^{-1} \mathrm{~d} g$ is gauge equivalent to the global connection form $A_{(0)}$ on $^{8}(\mathbb{C} \backslash\{0\}) / \mathbb{Z}_{n}$, where $g=\mathrm{e}^{\mathrm{i} p \varphi}$ is a large gauge transformation on this circle (i.e. not homotopic to the identity if $p \neq 1$ ). This is another manifestation of the fact that $m \in \mathbb{Z}_{n}$.

With this local model to hand, it is now straightforward to glue two ${ }^{9}$ copies to obtain a spindle. Associated with the north and south poles, we take local models $\left(\mathbb{C} \times S^{1}\right) / \mathbb{Z}_{n_{N}}$ and bundle data specified by $m_{N} \in \mathbb{Z}_{n_{N}}$, along with $\left(\mathbb{C} \times S^{1}\right) / \mathbb{Z}_{n_{S}}$ and bundle data specified by $m_{S} \in \mathbb{Z}_{n_{S}}$, respectively. The metrics in each patch on the total space of the bundle are as in (2.12) and given by

$$
\begin{aligned}
\mathrm{d} s_{\left(\mathbb{C} \times S^{1}\right) / \mathbb{Z}_{n_{N}}}^{2} & =\left(\mathrm{d} \psi_{N}+A_{(0)}^{N}+\frac{m_{N}}{n_{N}} \mathrm{~d} \varphi\right)^{2}+\mathrm{d} \rho_{N}^{2}+h\left(\rho_{N}\right)^{2} \frac{1}{n_{N}^{2}} \mathrm{~d} \varphi^{2}, \\
\mathrm{~d} s_{\left(\mathbb{C} \times S^{1}\right) / \mathbb{Z}_{n_{S}}}^{2} & =\left(\mathrm{d} \psi_{S}+A_{(0)}^{S}+\frac{m_{S}}{n_{S}} \mathrm{~d} \varphi\right)^{2}+\mathrm{d} \rho_{S}^{2}+h\left(\rho_{S}\right)^{2} \frac{1}{n_{S}^{2}} \mathrm{~d} \varphi^{2},
\end{aligned}
$$

where we may use the same name for the coordinate $\varphi$ in each patch, with $\Delta \varphi=2 \pi$, as this is identified in the gluing. Here $\psi_{N}, \psi_{S}$ each have period $2 \pi$, and $A_{(0)}^{N}, A_{(0)}^{S}$ are global oneforms in each of their respective covering space patches. The total gauge fields in each patch, $A^{N}=A_{(0)}^{N}+\frac{m_{N}}{n_{N}} \mathrm{~d} \varphi$ and $A^{S}=A_{(0)}^{S}+\frac{m_{S}}{n_{S}} \mathrm{~d} \varphi$, are defined only on an open disc in $\mathbb{C} \backslash\{0\}$, with $\rho_{N}, \rho_{S}>0$, as discussed above, with the flat connection capturing the orbibundle data. We glue on the circles at some fixed values of $\rho_{N}, \rho_{S}>0$, reversing the orientation of the south patch before gluing, where here $A^{N}$ and $A^{S}$ may in general differ by a U(1) gauge transformation $g: S_{\text {glue }}^{1} \rightarrow \mathrm{U}(1)$, of the form $g=\mathrm{e}^{\mathrm{i} p \varphi}$ with $p \in \mathbb{Z}$. That is, on the overlap

$$
A^{N}=A^{S}-\mathrm{i} g^{-1} \mathrm{~d} g=A^{S}+p \mathrm{~d} \varphi .
$$

The total gauge field flux $F=\mathrm{d} A$ through the spindle may then be computed using Stokes' theorem, being careful around the poles where the flat gauge fields are singular. This leads to an integral over two small circles around each pole, $S_{N, \epsilon}^{1}, S_{S, \epsilon}^{1}$, where $A_{N}^{(0)}$, $A_{S}^{(0)}$ are non-singular and hence give zero as $\epsilon \rightarrow 0$, but the flat gauge fields contribute.

\footnotetext{
${ }^{8}$ While $A=A_{(0)}$ is globally defined on $\mathbb{C} / \mathbb{Z}_{n}$, the large gauge transformation $g=\mathrm{e}^{\mathrm{i} p \varphi}$ is singular at the origin of $\mathbb{C}$.

${ }^{9}$ We can similarly glue an arbitrary number of such local models together using U(1) gauge transformations; the spindle is special in that we can consider metrics with azimuthal symmetry as we are interested in here. We also note that the total space of the $\mathrm{U}(1)$-orbibundles that we are constructing, when $\operatorname{hcf}(m, n)=1$ for all local models, give smooth 3-dimensional manifolds known as Seifert manifolds.
} 
We must also be careful where we glue over $S_{\text {glue }}^{1}$ and (2.33) holds. Thus we compute

$$
\begin{aligned}
\frac{1}{2 \pi} \int_{\Sigma} F & =-\frac{m_{N}}{n_{N}}+\frac{1}{2 \pi} \int_{S_{\text {glue }}^{1}} A^{N}-\left[-\frac{m_{S}}{n_{S}}+\frac{1}{2 \pi} \int_{S_{\text {glue }}^{1}} A^{S}\right] \\
& =p-\frac{m_{N}}{n_{N}}+\frac{m_{S}}{n_{S}} \equiv \frac{\lambda}{n_{N} n_{S}},
\end{aligned}
$$

where we have defined the integer $\lambda=-m_{N} n_{S}+m_{S} n_{N}+p n_{N} n_{S} \in \mathbb{Z}$. This shows that $\mathrm{U}(1)$-orbibundles over a spindle have gauge field flux given by $\lambda / n_{N} n_{S}$ with $\lambda \in \mathbb{Z}$. We refer to the associated complex line orbibundles as $\mathcal{O}(\lambda)$.

In fact $\lambda \in \mathbb{Z}$ specifies uniquely the orbibundle, up to isomorphism, and in particular it determines the local models near the orbifold points. To see this, note that using Bezout's Lemma it is immediate that for given $n_{N}, n_{S}$ it is possible to choose the $m_{N}, m_{S}$ with $p=0$ so that $\lambda=-m_{N} n_{S}+m_{S} n_{N}=1$. These $m_{N}, m_{S}$ are unique up to simultaneously shifting $m_{N} \mapsto m_{N}+k n_{N}, m_{S} \mapsto m_{S}+k n_{S}$, with $k \in \mathbb{Z}$, but this means that the mod reductions $m_{N} \in \mathbb{Z}_{n_{N}}, m_{S} \in \mathbb{Z}_{n_{S}}$ are unique, specifying the local model uniquely at each pole. Notice here that different choices of integer lifts $m_{N} \in \mathbb{Z}, m_{S} \in \mathbb{Z}$ in the $\operatorname{SL}(2, \mathbb{Z})$ transformation (2.11) may also then be absorbed into the choice of $p \in \mathbb{Z}$ in (2.16). Indeed, the gluing (2.16) may also be regarded as an $\operatorname{SL}(2, \mathbb{Z})$ transformation (see (2.20) below). Having discussed the case $\lambda=1$, other integer values for $\lambda$ may be obtained simply by multiplying $m_{N}, m_{S}$ by $\lambda$.

Notice from (2.16) that the non-singular gauge fields in each patch are related on the intersection by the gluing

$$
A_{(0)}^{N}=A_{(0)}^{S}+\frac{\lambda}{n_{N} n_{S}} \mathrm{~d} \varphi .
$$

While this characterization of the orbibundle on the spindle is in some sense equivalent to what we have just discussed, it is potentially confusing as one is gluing the gauge fields in each patch using fractional gauge transformations. By contrast, we reiterate that the gauge fields $A_{N}$ and $A_{S}$, with their flat connection pieces, are glued using U(1) gauge transformations on the overlap as in (2.16). Nevertheless, in previous papers discussing spindles we note that smooth gauges for the gauge fields have been used at the poles, with the corresponding fractional transition function in (2.18). While (2.18) is perfectly correct for a spindle, the discussion we have presented in this subsection is a precise global description of the construction and, moreover, also extends straightforwardly to more general orbifold Riemann surfaces.

\subsection{Spinors on spindles}

In the previous section we have "geometrized" the R-symmetry gauge field, introducing the $\mathrm{U}(1)$ fibre direction for which it is a corresponding connection. In the cases where the spindles arise in gauged supergravity, with an uplift to $D=10$ or $D=11$ dimensions, this is in any case part of the construction. We elaborate on this further in section 2.6

The total space of the U(1)-orbibundle is a Seifert fibred space, which is a smooth three-manifold $M_{3}$ when $\operatorname{hcf}(m, n)=1$ for all the local models. The one-form

$$
\eta \equiv \mathrm{d} \psi+A,
$$


is a smooth global one-form on this three-manifold. Focusing on the case of the spindle with two orbifold points, in the above construction we defined this in patches, and in particular on the overlap we have

$$
\psi_{N}=\psi_{S}-p \varphi
$$

which from (2.16) then ensures that $\mathrm{d} \psi_{N}+A^{N}=\mathrm{d} \psi_{S}+A^{S}$ on the overlap. This is precisely the $\mathrm{U}(1)$ gauge transformation $\mathrm{e}^{\mathrm{i} \psi_{N}}=\mathrm{e}^{\mathrm{i} \psi_{S}} \cdot \mathrm{e}^{-\mathrm{i} p \varphi}$. Near to the poles one should invert the $\mathrm{SL}(2, \mathbb{Z})$ transformation $(2.11)$ to obtain the connection one-forms $\mathrm{d} \chi_{N}+A_{(0)}^{N}, \mathrm{~d} \chi_{S}+A_{(0)}^{S}$, which are smooth one-forms on the covering copies of $\mathbb{C}$. Whenever one checks smoothness of local expressions in the orbifold setting, one necessarily has to resort to using patches and covering spaces in this way. We note in passing that the total space $M_{3}$ is a Lens space, when the orbifold base $\Sigma$ is a spindle.

By definition the Killing spinor $\zeta$ has charge $\frac{1}{2}$ under $A$. One can then introduce a complex line bundle $L$, on which $A$ is a connection, so that the spinor is a section of $S \otimes L^{1 / 2}$, where $S$ is the spin bundle on $\Sigma$. The vector field $\partial_{\psi}$ on $M_{3}$ generates the U(1) action, such that $\Sigma=M_{3} / \mathrm{U}(1)$ is the quotient. Moreover, for any function or more generally tensor field on $M_{3}$ we may Fourier expand in eigenmodes of $\mathcal{L}_{\partial_{\psi}}$. A function of charge $r$ under $\partial_{\psi}$, with corresponding phase $\mathrm{e}^{\mathrm{i} r} \psi$, is then, in its dependence on $\Sigma$, precisely a section of $L^{r}$. One thus obtains a spinor on $\Sigma$ of charge $\frac{1}{2}$ under $A$ by starting with a spinor field on $M_{3}$ that has charge $\frac{1}{2}$ under $\mathcal{L}_{\partial_{\psi}}$. Indeed, notice that the tangent bundle of $M_{3}$ naturally splits as $T_{\partial_{\psi}} \oplus T_{\Sigma}$, and the spinor bundle on $M_{3}$ then likewise splits as a tensor product of the spinor bundle $S$ on $\Sigma$ with a spinor on the circle fibres of $M_{3}$. A charge $\frac{1}{2}$ spinor under $\mathcal{L}_{\partial_{\psi}}$ then has phase $\mathrm{e}^{\mathrm{i} \psi / 2}$, which is the anti-periodic spin structure around the circle fibres. The resulting spinor field on $M_{3}$ is then a genuine spinor, rather than a spinor charged under a gauge field. This is precisely how supersymmetry is realized in consistent truncations to gauged supergravity. The comments above apply whether $\Sigma$ is smooth or an orbifold, as long as one uses the appropriate patches and covering spaces in the orbifold setting when gluing local descriptions together.

With these preliminary global comments to hand, as in the previous subsection we may now introduce local coordinate patches and covering spaces in order to describe the Killing spinor $\zeta$. We thus introduce coordinate patches $\mathcal{U}_{N} \cong \mathbb{C} / \mathbb{Z}_{n_{N}}$ and $\mathcal{U}_{S} \cong \mathbb{C} / \mathbb{Z}_{n_{S}}$, containing the north and south poles, respectively, and analyse how the spinor $\zeta$ behaves near to the poles, as well as how it patches together at the equator, where $\mathcal{U}_{N}$ and $\mathcal{U}_{S}$ overlap. We begin by considering $\mathcal{U}_{N}$, equipped with polar coordinates $\left(\rho_{N} \geq 0, \varphi\right)$ such that $\rho_{N}=0$ is the north pole. We may also introduce corresponding Euclidean coordinates $x_{N}+\mathrm{i} y_{N} \equiv \rho_{N} \mathrm{e}^{\mathrm{i} \varphi / n_{N}}$, identifying $\mathcal{U}_{N} \cong \mathbb{C} / \mathbb{Z}_{n_{N}}$. Recalling (2.14) and (2.3) we write the gauge field in this patch as a sum of a regular one-form plus a flat connection:

$$
A^{N}=a_{(0)}^{N}\left(\rho_{N}\right) \mathrm{d} \varphi+\frac{m_{N}}{n_{N}} \mathrm{~d} \varphi
$$

where $m_{N} \in \mathbb{Z}$. We demand that $a_{(0)}^{N}(0)=0$ to ensure that first term on the right hand side is a well-defined one-form at the origin of polar coordinates (where $\mathrm{d} \varphi$ is a singular one-form) and notice this condition fixes the residual gauge freedom in (2.4). 
Since we wish to describe spinors we introduce an orthonormal frame $e_{N}^{1}, e_{N}^{2}$, such that $e_{N}^{1}(0)=\mathrm{d} x_{N}, e_{N}^{2}(0)=\mathrm{d} y_{N}$ agrees with the usual Euclidean frame at the north pole. We use gamma matrices $\gamma_{1}=\sigma^{1}, \gamma_{2}=\sigma^{2}$, where $\sigma^{a}$ are Pauli matrices, and take the chirality operator to be $\gamma_{3} \equiv-\mathrm{i} \gamma_{1} \gamma_{2}=\sigma^{3}$. We may then write our spinor $\zeta$ in this patch and in the given frame in the form

$$
\zeta^{N}=\left(\begin{array}{c}
\zeta_{+}^{N} \\
\zeta_{-}^{N}
\end{array}\right)
$$

We will discuss the smoothness of $\zeta_{ \pm}^{N}$ at the north pole momentarily.

The frame $e_{N}^{1}, e_{N}^{2}$ is not invariant under $k=\partial_{\varphi}$, so the charge of the spinor $(2.22)$ under $k$ is not immediately manifest in the Euclidean frame. We can make it manifest by changing to a $k$-invariant frame, defined by

$$
\hat{e}^{1} \equiv \mathrm{d} \rho, \quad \hat{e}^{2} \equiv f(\rho) \mathrm{d} \varphi .
$$

This is an orthonormal frame for the metric (2.2), where note that near the north pole this is a polar coordinate frame with $\hat{e}^{1}=\mathrm{d} \rho_{N}, \hat{e}^{2}=\rho_{N} \mathrm{~d} \varphi / n_{N}+O\left(\rho_{N}^{2}\right)$. The two frames are related by the $\mathrm{U}(1) \cong \mathrm{SO}(2)$ rotation

$$
\left(\begin{array}{c}
\hat{e}^{1} \\
\hat{e}^{2}
\end{array}\right)=\left(\begin{array}{cc}
\cos \frac{\varphi}{n_{N}} & \sin \frac{\varphi}{n_{N}} \\
-\sin \frac{\varphi}{n_{N}} & \cos \frac{\varphi}{n_{N}}
\end{array}\right)\left(\begin{array}{c}
e_{N}^{1} \\
e_{N}^{2}
\end{array}\right)
$$

When vectors are rotated by an orthogonal matrix $L^{a}{ }_{b}=\exp \left(s^{a}{ }_{b}\right)$, spinors transform in the spin representation with $\tilde{L}=\exp \left(\frac{1}{4} s^{a b} \gamma_{a b}\right)$. Hence in the new frame (2.23) the spinor $\zeta^{N}$ reads

$$
\hat{\zeta}^{N}=\left(\begin{array}{c}
\mathrm{e}^{\mathrm{i} \varphi / 2 n_{N}} \zeta_{+}^{N} \\
\mathrm{e}^{-\mathrm{i} \varphi / 2 n_{N}} \zeta_{-}^{N}
\end{array}\right)
$$

Since the frame (2.23) is $k$-invariant, $\mathcal{L}_{k} \hat{e}^{a}=0$, we have $\mathcal{L}_{k} \hat{\zeta}^{N}=\partial_{\varphi} \hat{\zeta}^{N}$. Recall that in a fixed gauge of the form (2.3) we take the spinor to have definite charge under $k$. More precisely, in the patch $\mathcal{U}_{N}$ covering the north pole with gauge field (2.21) we take the charge of the spinor to be $Q_{N}$. It then follows that the spinor in the two frames takes the form

$$
\zeta^{N}=\left(\begin{array}{c}
\mathrm{e}^{\mathrm{i}\left(Q_{N}-\frac{1}{2 n_{N}}\right) \varphi} f_{+}^{N}\left(\rho_{N}\right) \\
\mathrm{e}^{\mathrm{i}\left(Q_{N}+\frac{1}{2 n_{N}}\right) \varphi} f_{-}^{N}\left(\rho_{N}\right)
\end{array}\right), \quad \hat{\zeta}^{N}=\mathrm{e}^{\mathrm{i} Q_{N} \varphi}\left(\begin{array}{c}
f_{+}^{N}\left(\rho_{N}\right) \\
f_{-}^{N}\left(\rho_{N}\right)
\end{array}\right) .
$$

We now return to the issue of smoothness at the north pole. If the spinor $\zeta$ were uncharged under the gauge field $A$, then by definition it is smooth at the north pole of the spindle precisely if its lift to the covering space $\mathbb{C}$, where $\varphi$ has period $2 \pi n_{N}$, is smooth at the origin $\rho_{N}=0$. The expression for $\zeta^{N}$ in (2.26) is with respect to a smooth frame at the origin, and thus the spinor will be regular precisely if its components are smooth functions at the origin. Notice now that if the positive chirality component $\zeta_{+}^{N}$ is non-zero at the origin, so $f_{+}^{N}(0) \neq 0$, then we must have $Q_{N}=1 / 2 n_{N}$ for $\zeta_{+}^{N}$ to be smooth, as $\varphi$ is 
singular at the origin. Similarly, if instead $\zeta_{-}^{N}$ is non-zero at the origin we would instead deduce that $Q_{N}=-1 / 2 n_{N}$.

However, the spinor $\zeta$ instead has charge $\frac{1}{2}$ under $A$ in the KSE as in (2.1), where in the north pole patch $A=A^{N}$ takes the form in (2.21). As discussed earlier in this subsection, this charged spinor $\zeta$ arises via reduction of a spinor on $M_{3}$ that has charge $\frac{1}{2}$ under $\mathcal{L}_{\partial_{\psi}}$. Moreover, to examine regularity of this spinor at the origin of $\mathbb{C}$ we must invert the $\mathrm{SL}(2, \mathbb{Z})$ transformation $(2.11)$, which removes the singular flat connection term $\frac{m_{N}}{n_{N}} \mathrm{~d} \varphi$ in the gauge field $A^{N}$. This leads to coordinates $\left(\rho_{N}, \hat{\phi}_{N}, \chi_{N}\right)$, with $\hat{\phi}_{N}$ and $\chi_{N}$ having period $2 \pi$ in the covering space, with the twisted $\mathbb{Z}_{n_{N}}$ action:

$$
\left(\chi_{N}, \hat{\phi}_{N}\right) \mapsto\left(\chi_{N}+\frac{2 \pi m_{N}}{n_{N}}, \hat{\phi}_{N}+\frac{2 \pi}{n_{N}}\right) .
$$

After uplifting the spinor $\zeta^{N}$ to $M_{3}$ we thus obtain

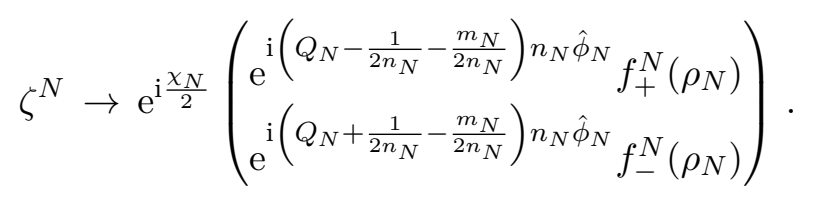

This is then smooth precisely if the components are smooth functions at $\rho_{N} \rightarrow 0$. As in the paragraph above, we then immediately deduce that if the positive chirality (upper) component is non-vanishing at the north pole then we must have $Q_{N}-\frac{1}{2 n_{N}}-\frac{m_{N}}{2 n_{N}}=0$, and similarly if the negative chirality (lower) component is non-vanishing at the north pole then we must have $Q_{N}+\frac{1}{2 n_{N}}-\frac{m_{N}}{2 n_{N}}=0$.

A similar analysis may be carried out in the south pole patch $\mathcal{U}_{S}$, equipped with polar coordinates $\left(\rho_{S} \geq 0, \varphi\right)$ such that $\rho_{S}=0$ is the south pole. The corresponding Euclidean coordinates are $x_{S}+\mathrm{i} y_{S} \equiv-\rho_{S} \mathrm{e}^{-\mathrm{i} \varphi / n_{S}}$, and we take

$$
A^{S}=a_{(0)}^{S}\left(\rho_{S}\right) \mathrm{d} \varphi+\frac{m_{S}}{n_{S}} \mathrm{~d} \varphi,
$$

where $m_{S} \in \mathbb{Z}$ and we demand that $a_{(0)}^{S}(0)=0$ to ensure that first term on the right hand side is smooth one-form at the origin of polar coordinates The only difference now is that the $k$-invariant frame (2.23) has $\hat{e}^{1}=-\mathrm{d} \rho_{S}, \hat{e}^{2}=\rho_{S} \mathrm{~d} \varphi / n_{S}+O\left(\rho_{S}^{2}\right)$. The analogous $\mathrm{U}(1) \cong \mathrm{SO}(2)$ rotation to $(2.24)$ reads

$$
\left(\begin{array}{l}
\hat{e}^{1} \\
\hat{e}^{2}
\end{array}\right)=\left(\begin{array}{cc}
\cos \frac{\varphi}{n_{S}} & -\sin \frac{\varphi}{n_{S}} \\
\sin \frac{\varphi}{n_{S}} & \cos \frac{\varphi}{n_{S}}
\end{array}\right)\left(\begin{array}{c}
e_{S}^{1} \\
e_{S}^{2}
\end{array}\right)
$$

where at the south pole we take $e_{S}^{1}(0)=\mathrm{d} x_{S}, e_{S}^{2}(0)=\mathrm{d} y_{S}$. With respect to the nonsingular frame $e_{S}^{a}$, and the $k$-invariant frame (2.23), a similar analysis to that at the north pole then leads to the respective spinors

$$
\zeta^{S}=\left(\begin{array}{c}
\mathrm{e}^{\mathrm{i}\left(Q_{S}+\frac{1}{2 n_{S}}\right) \varphi} \\
f_{+}^{S}\left(\rho_{S}\right) \\
\mathrm{e}^{\mathrm{i}\left(Q_{S}-\frac{1}{2 n_{S}}\right) \varphi} f_{-}^{S}\left(\rho_{S}\right)
\end{array}\right), \quad \hat{\zeta}^{S}=\mathrm{e}^{\mathrm{i} Q_{S} \varphi}\left(\begin{array}{c}
f_{+}^{S}\left(\rho_{S}\right) \\
f_{-}^{S}\left(\rho_{S}\right)
\end{array}\right)
$$


Also, after uplifting the spinor $\zeta^{S}$ to $M_{3}$ we obtain

$$
\zeta^{S} \rightarrow \mathrm{e}^{\mathrm{i} \frac{\chi_{S}}{2}}\left(\begin{array}{c}
\mathrm{i}\left(Q_{S}+\frac{1}{2 n_{S}}-\frac{m_{S}}{2 n_{S}}\right) n_{S} \hat{\phi}_{S} \\
f_{+}^{S}\left(\rho_{S}\right) \\
\mathrm{e}^{\mathrm{i}\left(Q_{S}-\frac{1}{2 n_{S}}-\frac{m_{S}}{2 n_{S}}\right) n_{S} \hat{\phi}_{S}} f_{-}^{S}\left(\rho_{S}\right)
\end{array}\right),
$$

with the components smooth functions at $\rho_{S} \rightarrow 0$.

Although the hatted spinors $\hat{\zeta}^{N}, \hat{\zeta}^{S}$ are defined using the same frame (2.23), note that the former uses the gauge field $A^{N}$, while the latter uses the gauge field $A^{S}$. As in (2.33) these gauge fields are related by a $\mathrm{U}(1)$ gauge transformation:

$$
A^{N}=A^{S}-\mathrm{i} g^{-1} \mathrm{~d} g=A^{S}+p \mathrm{~d} \varphi,
$$

with $p \in \mathbb{Z}$. Since the Killing spinor has charge $\frac{1}{2}$ under $A$, we correspondingly have the relation

$$
\hat{\zeta}^{N}=\mathrm{e}^{\mathrm{i}(p / 2) \varphi} \hat{\zeta}^{S} .
$$

This is satisfied by the expressions in $(2.26),(2.31)$ provided that $f_{ \pm}^{N}\left(\rho_{N}\right)=f_{ \pm}^{S}\left(\rho_{S}\right)$ on the overlap, as well as

$$
Q_{N}=Q_{S}+\frac{p}{2}
$$

With these results in hand, we may now investigate the consequences of our assumption (ii), namely that the spinor is non-zero at the poles. At the north pole at least one of the chiral components must be non-zero. Let us assume for the moment that $\zeta_{+}^{N}(0) \neq 0$, i.e. $f_{+}^{N}(0) \neq 0$. Then from (2.28), as already discussed regularity of the spinor $\zeta^{N}$ at the north pole implies

$$
Q_{N}=\frac{1}{2 n_{N}}+\frac{m_{N}}{2 n_{N}} .
$$

The negative chirality component of the uplifted spinor is then $\mathrm{e}^{\mathrm{i} \chi_{N} / 2} \mathrm{e}^{\mathrm{i} \varphi / n_{N}} f_{-}^{N}\left(\rho_{N}\right)$, and for this to be regular at the north pole we must have $f_{-}^{N}\left(\rho_{N}\right)=0$ so that the spinor is necessarily chiral at the pole. In fact we shall see in specific examples that $f_{-}^{N}\left(\rho_{N}\right)$ vanishes linearly in $\rho_{N}$, near to $\rho_{N}=0$, which implies that to leading order near the pole $\mathrm{e}^{-\mathrm{i} \chi_{N} / 2} \zeta_{-}^{N} \sim z_{N} \equiv \rho_{N} \mathrm{e}^{\mathrm{i} \varphi / n_{N}}$, which is smooth.

Looking next at the south pole, there are two possibilities that follow from regularity of $\zeta^{S}$ :

$$
\begin{aligned}
\text { Twist : } & \zeta_{+}^{S}(0) \neq 0 \Rightarrow Q_{S}=-\frac{1}{2 n_{S}}+\frac{m_{S}}{2 n_{S}}, \\
\text { Anti-twist : } & \zeta_{-}^{S}(0) \neq 0 \quad \Rightarrow \quad Q_{S}=+\frac{1}{2 n_{S}}+\frac{m_{S}}{2 n_{S}} .
\end{aligned}
$$

Notice that similarly to the north pole, if $\zeta_{ \pm}^{S}(0) \neq 0$ then correspondingly $\zeta_{\mp}^{S}(0)=0$.

We now recall the formula for the total flux (2.17). Using (2.35) we can rewrite this as $\frac{\lambda}{n_{N} n_{S}}=2\left(Q_{N}-Q_{S}\right)-\frac{m_{N}}{n_{N}}+\frac{m_{S}}{n_{S}}$ and hence using $(2.36),(2.37)$ we deduce that

Twist : $\quad \lambda=n_{S}+n_{N}$,

Anti-twist : $\quad \lambda=n_{S}-n_{N}$. 
These are precisely the two possibilities (1.1), and imply that the gauge field $A$ is a connection on $\mathcal{O}\left(n_{S} \pm n_{N}\right)$, respectively. In the twist case, with the upper sign, this is the tangent bundle of the spindle $\Sigma=\mathbb{W} \mathbb{C P}_{\left[n_{S}, n_{N}\right]}^{1}$.

In reaching this conclusion we assumed that $\zeta_{+}^{N}(0) \neq 0$. If instead $\zeta_{-}^{N}(0) \neq 0$, a similar analysis leads to the two cases

$$
\lambda=-n_{S} \mp n_{N} .
$$

These are the twist and anti-twist cases (2.38), but with the opposite overall sign for the gauge field. Such solutions will be related to twist and anti-twist solutions by charge conjugation, in the following sense. Recall that we took the gamma matrices to be $\gamma_{1}=\sigma^{1}$, $\gamma_{2}=\sigma^{2}$. The charge conjugation matrix ${ }^{10}$ is then $B \equiv \sigma^{1}$, which satisfies $B^{-1} \gamma_{a} B=\gamma_{a}^{*}$, where we define the charge conjugate spinor as

$$
\zeta^{c} \equiv B \zeta^{*}=\left(\begin{array}{c}
\zeta_{-}^{*} \\
\zeta_{+}^{*}
\end{array}\right)
$$

If $\zeta$ satisfies (2.1), then it is straightforward to check that $\zeta^{c}$ satisfies the same equation with $A$ replaced by $A^{c} \equiv-A$ and $\mathcal{M}$ replaced by $\mathcal{M}^{c}$, where $\mathcal{M}^{*}=B^{-1} \mathcal{M}^{c} B$. Since our analysis did not depend on the precise form of $\mathcal{M}$, the solutions with $\lambda$ given by (2.39) may equivalently be viewed as twist and anti-twist solutions (2.38), but solving the Killing spinor equation with $\mathcal{M}$ replaced by $\mathcal{M}^{c}$. It might be the case that there is a $\mathbb{Z}_{2}$ action on the fields such that $\mathcal{M}^{c}$ is the same function of the transformed fields as $\mathcal{M}$, i.e. charge conjugation leaves the Killing spinor equation (2.1) invariant. However, either way one can effectively reduce to the two cases in (2.38).

The above analysis showed that the spinor is necessarily chiral at each of the two poles. The twist case is then compatible with having $\zeta_{-} \equiv 0$, so that the spinor is positive chirality. This is the case for the topological twist, where the spinor is chiral and moreover constant. On the other hand, for the anti-twist the spinor $\zeta$ is necessarily non-chiral, with opposite chirality components vanishing at the two poles.

It is illuminating to consider the global structure of $\operatorname{spin}^{c}$ bundles we have constructed above. Recall that on any oriented two-manifold or orbifold, chiral spinors are sections of the bundles

$$
S^{+}=K^{1 / 2}, \quad S^{-}=K^{-1 / 2}=\Lambda^{0,1} \otimes K^{1 / 2} .
$$

Here $K=\Lambda^{1,0}$ is the canonical bundle, namely the cotangent bundle, and $\Lambda^{0,1}$ denote $(0,1)$-forms with respect to the canonical complex structure, specified by the orientation. For the spindle $K=\mathcal{O}\left(-n_{S}-n_{N}\right)$, which notice has a genuine square root only when $n_{S}+n_{N}$ is even. On the other hand, our Killing spinor is also charged under $A$, which is a connection on the line bundle $L \equiv \mathcal{O}(\lambda)$. Since the spinor has charge $\frac{1}{2}$, the chiral

\footnotetext{
${ }^{10}$ We can also run the same argument that follows by replacing $B$ with $\tilde{B} \equiv B \gamma_{3}$, which satisfies $\tilde{B}^{-1} \gamma_{a} \tilde{B}=-\gamma_{a}^{*}$.
} 
components $\zeta_{ \pm}$of $\zeta$ are globally sections of the bundles

$$
\begin{aligned}
\zeta_{+}: S^{+} \otimes L^{1 / 2} & =\mathcal{O}\left(-\frac{1}{2}\left(n_{S}+n_{N}\right)\right) \otimes \mathcal{O}\left(\frac{1}{2}\left(n_{S} \pm n_{N}\right)\right) \\
& = \begin{cases}\mathcal{O}(0) & \text { Twist } \\
\mathcal{O}\left(-n_{N}\right) & \text { Anti-twist }\end{cases} \\
\zeta_{-}: S^{-} \otimes L^{1 / 2} & =\mathcal{O}\left(\frac{1}{2}\left(n_{S}+n_{N}\right)\right) \otimes \mathcal{O}\left(\frac{1}{2}\left(n_{S} \pm n_{N}\right)\right) \\
& = \begin{cases}\mathcal{O}\left(n_{S}+n_{N}\right) & \text { Twist } \\
\mathcal{O}\left(n_{S}\right) & \text { Anti-twist }\end{cases}
\end{aligned}
$$

Notice that these are well-defined line bundles over the spindle, even though in general neither $S^{ \pm}$nor $L^{1 / 2}$ is. This is a consequence of the fact that $\lambda=n_{S} \pm n_{N} \equiv n_{S}+n_{N}$ mod 2, meaning that $A$ is a $\operatorname{spin}^{c}$ gauge field. In the twist case notice that $\zeta_{+}$is a section of the trivial line bundle $\mathcal{O}(0)$.

Before continuing, we briefly pause to contrast the detailed global analysis for the regularity of the $\operatorname{spin}^{c}$ spinors that we have just carried out with what has appeared in the literature in the context of specific solutions [1-8]. In essence, these papers used patches with regular gauge fields $A_{(0)}^{N}, A_{(0)}^{S}$ that are related by fractional gauge transformations as in (2.18). This is then combined with demanding smoothness of the unhatted spinors given in (2.26), rather than (2.28). While this approach does in fact give the correct results for a spindle, our new discussion here provides a precise global description.

\subsection{Topological twist}

The "topological twist" is a particular case of the twist solutions. Specifically, one solves the original Killing spinor equation (2.1) by taking $\zeta_{-} \equiv 0$, so that $\zeta=\zeta_{+}$is a chiral spinor. Since then $\gamma_{12} \zeta=\mathrm{i} \sigma^{3} \zeta=\mathrm{i} \zeta$, equation (2.1) reads ${ }^{11}$

$$
\mathrm{d} \zeta+\frac{\mathrm{i}}{2}\left(\omega_{12}-A\right) \zeta+\mathcal{M} \zeta=0 .
$$

Assuming that $\mathcal{M} \zeta=0$, this is solved by taking

$$
A=\omega_{12}, \quad \zeta=\text { constant } .
$$

Taking $A$ to be the spin connection $\omega_{12}$ immediately implies that this is a connection on the tangent bundle of $\Sigma$, and hence (1.1) holds with the upper sign. Moreover, $\zeta=\zeta_{+}$ being nowhere zero is then necessarily a section of a trivial line bundle $\mathcal{O}(0)$. This is clearly a special case of what we have called "twist" solutions in the previous subsection.

However, more generally there are twist solutions that are not "topological twists" in the above strict sense, as seen in [6]. We will later see new examples of twist solutions with $\zeta_{-}$not identically zero (but necessarily zero at the poles of the spindle), and similarly

\footnotetext{
${ }^{11}$ If instead $\zeta=\zeta_{-}$has negative chirality, one instead takes $A=-\omega_{12}$, which is the upper sign solution in (2.39) for which $A$ is a connection on the cotangent bundle of $\Sigma$, rather than tangent bundle.
} 
$\mathcal{M} \zeta \neq 0$ and $\zeta_{+}$non-constant. We thus find it convenient to distinguish between topological twist and twist, although this distinction is not always made in the literature.

Finally, we note here that for the topological twist case we immediately deduce that the vector bilinears vanish: $\zeta^{\dagger} \gamma_{a} \zeta=\zeta^{\dagger} \gamma_{a} \gamma_{3} \zeta=0$. This is true not just for the two-sphere but also for a topological twist on an arbitrary Riemann surface. By contrast, as we will see later in explicit examples, for the twist case on a spindle there is a vector bilinear constructed from the Killing spinors which gives the Killing vector of the spindle. This demonstrates, at the level of gauged supergravity, that supersymmetry for the topological twist and the twist on the spindle are realized in quite distinct ways.

\subsection{Nowhere zero spinors}

The analysis so far was quite general, and apart from $k=\partial_{\varphi}$ generating a symmetry of the solution, the only other assumption we made was that the Killing spinor $\zeta$ is non-zero at the poles of the spindle. In fact in almost all cases of interest $\zeta$ is nowhere zero, as we now briefly discuss.

Starting with a non-trivial solution to the Killing spinor equation (2.1), we can follow an argument given in [12]. Assume that $\zeta\left(x_{0}\right)=0$ for some point $x_{0}$. Assuming the manifold is path-connected we can connect to any other point by a smooth curve $x(s)$. Along this curve (2.1) has the schematic form $\frac{\mathrm{d}}{\mathrm{d} s} \zeta(x(s))=\tilde{M}(s) \zeta(x(s))$ for a smooth matrix $\tilde{M}$. Invoking uniqueness of first order linear ODEs, with $\zeta\left(x_{0}\right)=0$ we deduce that $\zeta(x(s))=0$ and hence $\zeta \equiv 0$ identically, which contradicts our assumption of non-triviality. Thus, we deduce that $\zeta$ is nowhere vanishing.

This result is immediately applicable to $\operatorname{AdS} \times \Sigma$ solutions $^{12}$ of gauged supergravity. After uplifting to string theory and M-theory the supergravity metric takes the general form

$$
\mathrm{d} s^{2}=\mathrm{e}^{2 \Delta} \mathrm{d} s_{\text {AdS }}^{2}+\mathrm{d} s_{\text {internal }}^{2},
$$

where the warp factor $\Delta$ is a function of the internal space coordinates, so as to preserve the symmetries of AdS. The argument given in the preceding paragraph can in fact be immediately adapted to arbitrary supersymmetric solutions of the form (2.45), utilizing the Killing spinor equations for $D=10,11$ supergravity. In particular one can conclude that the internal space Killing spinor $\eta$ is nowhere vanishing. In particular, for solutions where $\mathrm{d} s_{\text {internal }}^{2}$ contains a spindle, the restriction of $\eta$ to the spindle is also nowhere zero.

As somewhat of an aside it is interesting to note that in many cases the internal space Killing spinor $\eta$ for such supersymmetric solutions satisfies

$$
\bar{\eta} \eta=\mathrm{e}^{\Delta},
$$

which implies that $\eta$ is nowhere zero. For example, the equation (2.46) holds for general supersymmetric $\mathrm{AdS}_{3}$ D3-brane solutions and M2-brane solutions i.e. $\mathcal{N}=(2,0) \mathrm{AdS}_{3}$

\footnotetext{
${ }^{12}$ The AdS $\times D$ solutions of e.g. $[8,13-16]$, where $D$ is topologically a disc, are constructed from the same family of local solutions that we are considering in this paper. In these solutions the Killing spinors vanish as one approaches the boundary of the disc. However, since this is a boundary, in fact a singular boundary (since the warp factor vanishes), our argument does not apply.
} 
solutions of type IIB with five-form flux and $\mathcal{N}=2 \mathrm{AdS}_{2}$ solutions of $D=11$ supergravity with electric four-form flux, respectively. The same formula holds for the most general supersymmetric $\mathrm{AdS}_{5}$ solutions of $D=11$ supergravity [17], and for the most general $\mathcal{N}=2 \mathrm{AdS}_{4}$ solutions of $D=11$ [18]. A similar conclusion is reached for the $\mathcal{N}=(1,0)$ $\mathrm{AdS}_{3}$ solutions of type IIB studied in [19].

The analysis in the previous subsections can also be applied to rigid supersymmetric backgrounds that contain a spindle. For supersymmetric backgrounds of so-called new minimal supergravity in $d=3$ and $d=4$ dimensions, the Killing spinors are necessarily nowhere zero - see, e.g. [12] and [20], respectively, using the above argument. Localization results for supersymmetric field theories with four supercharges in three and four dimensions have mainly been derived in this setting. However, we note that if one uses conformal supergravity the spinor can have zeroes $;^{13}$ it would be interesting to know if supersymmetry on a spindle can be realised in this specific context in a manner that is different from the twist and the anti-twist. Furthermore, it would also be of interest to know if such an alternative does exist, then could it ever arise with the rigid background arising as the conformal boundary of an AdS solution.

\subsection{Uplifted solutions}

Let us return to the general formula (2.17) for the flux of the gauge field strength through the spindle. For $\lambda=n_{S} \pm n_{N}$ in the twist and anti-twist cases, respectively, we may solve this by taking $p=0$ and $m_{N}=-1, m_{S}= \pm 1$. As discussed after equation (2.17) these choices are not unique, but $m_{N} \in \mathbb{Z}_{n_{N}}, m_{S} \in \mathbb{Z}_{n_{S}}$ are unique, and this is the invariant data for the local models at each pole. Moreover, notice that $m=\mp 1$ precisely give the tangent and cotangent bundle actions at a pole: if the generator $\omega$ of $\mathbb{Z}_{n}$ acts as $z \mapsto \omega z$ on $\mathbb{C}$, then the one-form $\mathrm{d} z \mapsto \omega \mathrm{d} z$, while the tangent vector $\partial_{z} \mapsto \omega^{-1} \partial_{z}$. With the above choices we then have that

$$
\tilde{A} \equiv A^{N}=A^{S}
$$

is a global one-form on the spindle minus both of its poles. We may also then write this near each pole as

$$
\tilde{A}=A_{(0)}^{N}-\frac{1}{n_{N}} \mathrm{~d} \varphi=A_{(0)}^{S} \pm \frac{1}{n_{S}} \mathrm{~d} \varphi,
$$

where recall that $A_{(0)}^{N}$ and $A_{(0)}^{S}$ are smooth one-forms in their respective patches, and in particular smooth at each pole, respectively. It then follows that $\tilde{A}$ is singular at both poles, where from (2.48) we deduce

$$
\left.\tilde{A}\right|_{N}=-\frac{1}{n_{N}} \mathrm{~d} \varphi,\left.\quad \tilde{A}\right|_{S}=\left\{\begin{array}{ll}
+\frac{1}{n_{S}} \mathrm{~d} \varphi & \text { Twist } \\
-\frac{1}{n_{S}} \mathrm{~d} \varphi & \text { Anti-twist }
\end{array} .\right.
$$

\footnotetext{
${ }^{13}$ To see why the above argument can fail, consider the conformal Killing spinor equation $\left(\delta_{\mu}^{\nu}-\right.$ $\left.\frac{1}{d} \gamma_{\mu} \gamma^{\nu}\right) \nabla_{\nu} \zeta=\ldots$, where the right hand side could contain other fields, and notice that $\left(\delta_{\mu}^{\nu}-\frac{1}{d} \gamma_{\mu} \gamma^{\nu}\right)$ is a projection.
} 
We shall make use of this gauge for the field theory analysis later in the paper. In particular, from (2.36) and (2.37) we see that $Q_{N}=Q_{S}=0$, and the spinor $\zeta$ is uncharged in this gauge.

Recall that in sections 2.2 and 2.3 we "geometrized" the R-symmetry gauge field by introducing a corresponding circle direction $\psi$, where after appropriate gluing one verifies that $\eta=\mathrm{d} \psi+A$ is a global one-form on the total space $M_{3}$. This precisely happens when the Killing spinor equation (2.1) arises by dimensionally reducing a $D=10$ or $D=11$ Killing spinor equation, associated with a solution of the form (2.45). On uplifting, the Abelian R-symmetry gauge field $A$ becomes a connection in the internal space metric, with the gauge transformations we have discussed becoming coordinate transformations. The Killing vector $\partial_{\psi}$ then generates an isometry of the internal space. For example, to be concrete we may consider the original D3-brane spindle solutions in [1]. These are $\mathrm{AdS}_{3} \times Y_{7}$ solutions of type IIB supergravity, where $Y_{7}$ is the total space of a regular Sasaki-Einstein 5-manifold $S E_{5}$ fibred over a spindle $\Sigma$. Here $S E_{5}$ by definition itself fibres over a Kähler-Einstein 4-manifold $K E_{4}$, and fixing a point in the later space inside $Y_{7}$ is precisely our (Lens space) $M_{3}$, with $\psi$ being fibred both over the $K E_{4}$ and also over the spindle. The fibration over the $K E_{4}$ doesn't play any role in the regularity analysis we have been discussing thus far.

On the other hand, in the lifted geometry one can phrase things more globally and invariantly. The $D=10$ or $D=11$ Killing spinor will form a representation of the isometry group, and if there is a single complex spinor then this statement means it will have definite charge $Q_{i}$ under the $i$ th $\mathrm{U}(1)$ isometry $\partial_{\varphi_{i}}$. Generically, in the examples discussed in this paper, there are at least two such isometries. However, we can always choose a basis in which the spinor has charge $\frac{1}{2}$ under $\partial_{\varphi_{1}} \equiv \partial_{\psi}$, and is uncharged under $\partial_{\varphi_{2}}$. The latter is by definition then a flavour isometry. The gauge $\tilde{A}$ for $A$ in (2.48) is then precisely a choice of gauge for which $\partial_{\varphi}=\partial_{\varphi_{2}}$ is a flavour symmetry.

In the known examples of the anti-twist and twist on the spindle, as highlighted at the end of section 2.4, there is a vector bilinear for the Killing spinors of the gauged supergravity solution that involves the Killing direction on the spindle. After uplifting, one finds this implies that there is a vector bilinear for the higher-dimensional Killing spinors which is a linear combination of this Killing vector with the Killing vector $\partial_{\psi}$. The dual interpretation of this is that the lower-dimensional SCFT has an R-symmetry that is a linear combination of the rotational symmetry on the spindle with the R-symmetry of the higher-dimensional SCFT upon which the SCFT is wrapped.

\section{D3-branes}

We now re-examine the case of D3-branes wrapping spindles that was considered in $[1,4,5]$. For the cases associated with solutions of minimal $D=5$ gauged supergravity we recover exactly the same results as [1] where it was shown, in particular, that only the anti-twist case is allowed. For the cases associated with the $D=5$ STU gauged supergravity we will recover the results of $[4,5]$ for the anti-twist case, but we will show here, as a new result, that the twist case is also possible. In contrast to the anti-twist solutions, these new twist solutions are naturally interpreted as the near-horizon limits of D3-branes wrap- 
ping a spindle embedded as a holomorphic curve inside a Calabi-Yau four-fold. Moreover, taking a (formal) limit of the central charge for these twist solutions, in which the spindle becomes a two-sphere, we reproduce the central charge of the topologically twisted solutions of [21]. We will also discuss how a special sub-class of solutions are associated with M5-branes wrapped on the product of a spindle with a Riemann surface. We also make some comparisons with field theory.

\subsection{The $D=5$ gauged supergravity model}

The $D=5$ STU, $\mathrm{U}(1)^{3}$ gauged supergravity model that we shall consider has a Lagrangian given by (see e.g. [22])

$$
\begin{aligned}
\mathcal{L}= & \sqrt{-g}\left[R-\mathcal{V}-\frac{1}{2} \sum_{I=1}^{3}\left(X^{(I)}\right)^{-2}\left(\partial X^{(I)}\right)^{2}-\frac{1}{4} \sum_{I=1}^{3}\left(X^{(I)}\right)^{-2}\left(F^{(I)}\right)^{2}\right] \\
& -F^{(1)} \wedge F^{(2)} \wedge A^{(3)}
\end{aligned}
$$

where $A^{(I)}$ are three $\mathrm{U}(1)$ gauge fields, $I=1,2,3$, with field strengths $F^{(I)}=\mathrm{d} A^{(I)}$. The three scalar fields $X^{(I)}>0$ satisfy the constraint $X^{(1)} X^{(2)} X^{(3)}=1$ and can be written in terms of two canonically normalized scalars $\varphi_{1}, \varphi_{2}$ via

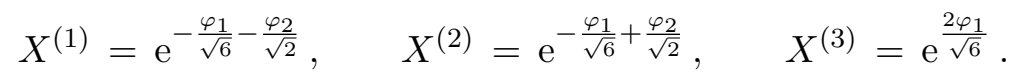

The potential is given by

$$
\mathcal{V}=-4 \sum_{I=1}^{3}\left(X^{(I)}\right)^{-1}
$$

The model is a consistent truncation of $D=5, \mathcal{N}=2$ gauged supergravity coupled to two vector multiplets. For a bosonic solution to preserve supersymmetry, we require that the variation of the gravitino and the two gauginos both vanish

$$
\begin{aligned}
0 & =\left[\nabla_{\mu}-\frac{\mathrm{i}}{2} \sum_{I=1}^{3} A_{\mu}^{(I)}+\frac{1}{6} \sum_{I=1}^{3} X^{(I)} \Gamma_{\mu}+\frac{\mathrm{i}}{24} \sum_{I=1}^{3}\left(X^{(I)}\right)^{-1}\left(\Gamma_{\mu}{ }^{\nu \rho}-4 \delta_{\mu}^{\nu} \Gamma^{\rho}\right) F_{\nu \rho}^{(I)}\right] \epsilon, \\
0 & =\left[\not \partial \varphi_{i}-2 \sum_{I=1}^{3} \partial_{\varphi_{i}} X^{(I)}+\frac{\mathrm{i}}{2} \sum_{I=1}^{3} \partial_{\varphi_{i}}\left(X^{(I)}\right)^{-1} F_{\mu \nu}^{(I)} \Gamma^{\mu \nu}\right] \epsilon,
\end{aligned}
$$

where $\Gamma^{\mu}$ are $D=5$ Gamma matrices, $\nabla=\mathrm{d}+\frac{1}{4} \omega^{a b} \Gamma_{a b}$, with $\omega^{a b}$ the Levi-Civita connection, and $\epsilon$ is a Dirac spinor. We can define the R-symmetry gauge field via

$$
A^{R} \equiv \sum_{I=1}^{3} A^{(I)},
$$

which is then normalized as in section 2 .

Any supersymmetric solution of this model can be uplifted on $S^{5}$ to give a supersymmetric solution of type IIB supergravity using the results of [23]. ${ }^{14}$ In particular, the

\footnotetext{
${ }^{14}$ As pointed out in [10] there is a typo in the five-form flux in [23]. The corrected expression may be found in [5].
} 
vacuum $\mathrm{AdS}_{5}$ solution, which has unit radius, uplifts to the $\mathrm{AdS}_{5} \times S^{5}$ solution, dual to $d=4 \mathcal{N}=4 \mathrm{SYM}$ theory. In the special case that two gauge fields are equal, and also reducing to one scalar field, for example $A^{(1)}=A^{(2)}$ and $X^{(1)}=X^{(2)}$ (i.e. $\varphi_{2}=0$ ), we can then uplift on a class of $M_{6}$ geometries that arise in the most general $\mathrm{AdS}_{5} \times M_{6}$ solutions of $D=11$ supergravity dual to $\mathcal{N}=2$ SCFTs [24], to give supersymmetric solutions of $D=11$ supergravity [25]. In particular, this includes uplifting on $M_{6}$ which are an $S^{4}$ fibration over a Riemann surface $\Sigma_{g}$ with genus $g>1$, that arise in the Maldacena-Núñez solution describing M5-branes wrapped on a Riemann surface embedded in a Calabi-Yau two-fold [11], or more generally a punctured Riemann surface as in [26]. Setting three gauge fields equal and the scalars to zero leads to minimal gauged supergravity, and the solutions can be uplifted to type IIB on a Sasaki-Einstein manifold [27]; this case is discussed in [1].

\subsection{Supergravity solution and global analysis}

We consider the supersymmetric solutions of $D=5$ STU gauged supergravity that were presented in [28], and then recently analysed in $[4,5]$. Following ${ }^{15}[5]$, the local form of the solutions is given by

$$
\begin{aligned}
& \mathrm{d} s_{5}^{2}=H^{1 / 3}\left[\mathrm{~d} s_{\mathrm{AdS}_{3}}^{2}+\frac{1}{4 P} \mathrm{~d} y^{2}+\frac{P}{H} \mathrm{~d} z^{2}\right]=H^{1 / 3}\left[\mathrm{~d} s_{\mathrm{AdS}_{3}}^{2}+\mathrm{d} s_{\Sigma}^{2}\right], \\
& A^{(I)}=\frac{y}{h_{I}} \mathrm{~d} z, \quad X^{(I)}=\frac{H^{1 / 3}}{h_{I}},
\end{aligned}
$$

where $\mathrm{d} s_{\mathrm{AdS}_{3}}^{2}$ is a unit radius metric and $h_{I}, H$ and $P$ are all functions of $y$ given by

$$
h_{I}=y+q_{I}, \quad H=\prod_{I=1}^{3} h_{I}, \quad P=H-y^{2},
$$

and $q_{I}, I=1,2,3$ are constants. Note that the field strength can be written as

$$
\left(X^{(I)}\right)^{-2} F^{(I)}=\frac{2 q_{I}}{H^{1 / 2}} \widetilde{\operatorname{vol}}_{\Sigma}
$$

where $\widetilde{\operatorname{vol}}(\Sigma)$ is the volume form associated to the conformally rescaled spindle metric $\widetilde{\mathrm{d} s^{2}}{ }_{\Sigma}=H^{1 / 3} \mathrm{~d} s_{\Sigma}^{2}$.

We want to choose the parameters so that $y, z$ parametrize a compact spindle and with a positive warp factor, $H>0$. Since $X^{(I)}>0$, we must have $h_{I}>0$. Also note that $P>0$ implies that $H>0$. We assume that $P$ has three real roots, which we take to be $y_{1}<y_{2}<y_{3}$, and we take $y \in\left[y_{1}, y_{2}\right]$ with $P \geq 0$. Expanding near the boundaries of this interval, after setting $\varrho_{i}=2\left|y-y_{i}\right|^{1 / 2}$ for $i=1,2$, we find

$$
\mathrm{d} s_{\Sigma}^{2} \simeq \frac{1}{4\left|P^{\prime}\left(y_{i}\right)\right|}\left(\mathrm{d} \varrho_{i}^{2}+\kappa_{i}^{2} \varrho_{i}^{2} \mathrm{~d} z^{2}\right), \quad \text { where } \quad \kappa_{i}=\left|\frac{P^{\prime}\left(y_{i}\right)}{y_{i}}\right|, \quad i=1,2 .
$$

We would now like to remove the absolute value in the definition of $\kappa_{i}$. From the expression for $P$ it is clear that $P^{\prime}\left(y_{1}\right)>0$ and $P^{\prime}\left(y_{2}\right)<0$ and so, with $y_{1}<y_{2}$, we need to distinguish

\footnotetext{
${ }^{15}$ We have slightly changed the parametrization of the solutions as compared to [5] in order to be more uniform with later sections.
} 
three cases corresponding to the three possible signs for $y_{i}(i=1,2)$. This is conveniently achieved by writing

$$
\eta_{1} y_{1}<0, \quad \eta_{2} y_{2}>0
$$

with the three cases determined by the signs

$$
\begin{array}{ll}
\text { Case A : } & \left(\eta_{1}, \eta_{2}\right)=(-1,+1), \\
\text { Case B : } & \left(\eta_{1}, \eta_{2}\right)=(+1,+1), \\
\text { Case C : } & \left(\eta_{1}, \eta_{2}\right)=(+1,-1) .
\end{array}
$$

Then, for all three cases we can write

$$
\kappa_{i}=-\frac{\eta_{i} P^{\prime}\left(y_{i}\right)}{y_{i}}
$$

and $\Sigma$ will be a spindle provided we impose

$$
\Delta z=\frac{2 \pi}{\kappa_{1} n_{1}}=\frac{2 \pi}{\kappa_{2} n_{2}},
$$

where $n_{1}, n_{2} \in \mathbb{N}$ with $\operatorname{hcf}\left(n_{1}, n_{2}\right)=1$. As we will see, only cases $\mathrm{A}$ and $\mathrm{B}$ will in fact be realized.

We next demand that the three gauge field fluxes on the spindle are suitably quantized. Specifically, as in [5] with the $D=5$ spinors carrying charge $\frac{1}{2}$ with respect to each of the gauge fields, we demand

$$
\begin{aligned}
Q_{I} & \equiv \frac{1}{2 \pi} \int_{\Sigma} F^{(I)}=\left(\frac{y_{2}}{h_{I}\left(y_{2}\right)}-\frac{y_{1}}{h_{I}\left(y_{1}\right)}\right) \frac{\Delta z}{2 \pi} \\
& \equiv \frac{p_{I}}{n_{1} n_{2}}, \quad p_{I} \in \mathbb{Z}
\end{aligned}
$$

After writing

$$
P=\left(y-y_{1}\right)\left(y-y_{2}\right)\left(y-y_{3}\right),
$$

and comparing with the expression for $P$ in (3.7), one can now solve (3.13) and (3.14) for $y_{1,2}, q_{1,2,3}$ and $\Delta z$ in terms of the spindle data $n_{1}, n_{2}$ and the quantized fluxes $p_{1,2,3}$. We will give the explicit solution below.

However, without knowing the explicit solution one can compute the Euler number of the spindle, $\chi(\Sigma)$. Using the metric

$$
\mathrm{d} s_{\Sigma}^{2}=\frac{1}{4 P} \mathrm{~d} y^{2}+\frac{P}{H} \mathrm{~d} z^{2},
$$

one finds $\sqrt{g}_{\Sigma} R_{\Sigma}=2\left[H^{-3 / 2}\left(P H^{\prime}-H P^{\prime}\right)\right]^{\prime}$ from which we calculate

$$
\chi(\Sigma)=\frac{1}{4 \pi} \int_{\Sigma} \sqrt{g}_{\Sigma} R_{\Sigma} \mathrm{d} y \mathrm{~d} z=\frac{\Delta z}{2 \pi}\left(\frac{P^{\prime}\left(y_{1}\right)}{\left|y_{1}\right|}-\frac{P^{\prime}\left(y_{2}\right)}{\left|y_{2}\right|}\right)=\frac{n_{1}+n_{2}}{n_{1} n_{2}},
$$

where we have used $H\left(y_{i}\right)=y_{i}^{2},(3.9)-(3.13)$, and the result is independent of which case is considered. Equivalently, using $\mathrm{e}^{1}=\mathrm{d} y /\left(2 P^{1 / 2}\right), \mathrm{e}^{2}=\left(P^{1 / 2} / H^{1 / 2}\right) \mathrm{d} z$ as an orthonormal 
frame, the spin connection for $\mathrm{d} s_{\Sigma}^{2}$ is given by $\omega_{\Sigma}^{12}=\left[H^{-3 / 2}\left(P H^{\prime}-H P^{\prime}\right)\right] \mathrm{d} z$. When evaluated at the roots we have

$$
\omega_{\Sigma}^{12}\left(y_{1}\right)=-\frac{\mathrm{d} \varphi}{n_{1}}, \quad \omega_{\Sigma}^{12}\left(y_{2}\right)=+\frac{\mathrm{d} \varphi}{n_{2}},
$$

and hence $\chi(\Sigma)=(2 \pi)^{-1} \int_{\Sigma} \mathrm{d} \omega_{\Sigma}^{12}$ gives the same result. Now $\omega_{\Sigma}^{12}$ is an $\mathrm{SO}(2) \cong \mathrm{U}(1)$ connection on the tangent bundle, and it is interesting to highlight that the behaviour at the poles given in (3.18) is in alignment with the comments in section 2.6.

In addition, we can also compute the total R-symmetry flux $Q^{R} \equiv\left(Q_{1}+Q_{2}+Q_{3}\right)$. To that end, one can use the identities

$$
\begin{aligned}
& P^{\prime}=h_{1} h_{2}+h_{2} h_{3}+h_{1} h_{3}-2 y, \\
& y_{i}^{2}=h_{1}\left(y_{i}\right) h_{2}\left(y_{i}\right) h_{3}\left(y_{i}\right), \quad(i=1,2),
\end{aligned}
$$

in (3.12), which allows us to rewrite (3.13) as

$$
\left.\frac{\Delta z}{2 \pi}\left[2-y_{i} \sum_{I=1}^{3} \frac{1}{h_{I}\left(y_{i}\right)}\right]=\frac{\eta_{i}}{n_{i}}, \quad \text { (no sum on } i\right) .
$$

One can now immediately prove that the R-symmetry flux is given by

$$
Q^{R}=\frac{\eta_{1} n_{2}-\eta_{2} n_{1}}{n_{1} n_{2}} \quad \Leftrightarrow \quad p_{1}+p_{2}+p_{3}=\eta_{1} n_{2}-\eta_{2} n_{1} .
$$

Case $\mathrm{B}$ is the anti-twist that was considered in $[4,5]$. We now see that the same local solutions can also potentially realize the twist in cases $\mathrm{A}$ and $\mathrm{C}$, although we will ultimately find that case $\mathrm{C}$ is not possible, while case $\mathrm{A}$ is.

The central charge for the $(0,2)$ SCFT dual to the $\mathrm{AdS}_{3} \times \Sigma$ solution can be expressed in the form

$$
c=\frac{3}{2 \pi} \Delta z\left(y_{2}-y_{1}\right) N^{2},
$$

as in [5]. To express this in terms of spindle data we now need to solve for $y_{1,2}$ and $\Delta z$ in terms of $n_{i}$ and $p_{1}, p_{2}$ (recall that $p_{3}$ is given by the constraint (3.21)), as outlined below (3.15). The explicit solution given in [5] is valid for case B. With a little effort one can rewrite the solution of [5] by reintroducing $p_{3}$ and then using the signs $\eta_{1,2}$ introduced in (3.11) to deduce expressions that are valid for all three cases. Specifically, for the constants $q_{I}$ we find

$$
\begin{aligned}
& q_{1}=\frac{8}{s^{3}} p_{2} p_{3}\left(n_{1}+\eta_{2} p_{2}\right)\left(n_{1}+\eta_{2} p_{3}\right)\left(n_{2}-\eta_{1} p_{2}\right)\left(n_{2}-\eta_{1} p_{3}\right), \\
& q_{2}=\left.q_{1}\right|_{p_{1} \leftrightarrow p_{2}} \\
& q_{3}=\left.q_{1}\right|_{p_{1} \leftrightarrow p_{3}}
\end{aligned}
$$

where we have introduced

$$
s \equiv n_{1}^{2}+n_{2}^{2}-\left(p_{1}^{2}+p_{2}^{2}+p_{3}^{2}\right) .
$$


The roots $y_{1,2}$ are given by

$$
\begin{aligned}
& y_{1}=-\frac{8 \eta_{2}}{s^{3}} p_{1} p_{2} p_{3}\left(n_{1}+\eta_{2} p_{1}\right)\left(n_{1}+\eta_{2} p_{2}\right)\left(n_{1}+\eta_{2} p_{3}\right), \\
& y_{2}=\left.y_{1}\right|_{n_{1} \leftrightarrow n_{2}, \eta_{2} \rightarrow-\eta_{1}},
\end{aligned}
$$

in terms of which $y_{3}$ is fixed using $\sum_{I=1}^{3}\left(y_{I}+q_{I}\right)=1$, and explicitly we have

$$
y_{3}=\frac{8 \eta_{1} \eta_{2}}{s^{3}} \prod_{I=1}^{3}\left(n_{1}+\eta_{2} p_{I}\right)\left(n_{2}-\eta_{1} p_{I}\right)
$$

Finally, we have

$$
\frac{\Delta z}{2 \pi}=\frac{s}{2 n_{1} n_{2}\left(\eta_{2} n_{1}+\eta_{1} n_{2}\right)} .
$$

With these results we can now express the central charge, for all three cases, in the simple form

$$
c=\frac{6 p_{1} p_{2} p_{3}}{n_{1} n_{2} s} N^{2}
$$

We now examine whether it is possible to choose the spindle data $n_{1,2}$ and quantized fluxes $p_{I}$, satisfying (3.21), such that cases A, B and C are in fact realized as bona fide solutions. Recall we have assumed from the start that $n_{1,2}>0$. We also need to check that

$$
h_{I}>0, \quad y_{1}<y_{2}<y_{3}, \quad \eta_{1} y_{1}<0, \quad \eta_{2} y_{2}>0
$$

where the first condition comes from the fact that the scalar fields $X^{(I)}>0$, and the last two come from (3.10). To analyse these constraints one can utilize the expressions given in (3.23), (3.25). Doing so, we find that case $\mathrm{C}$ is not possible, leaving just cases $\mathrm{A}$ and $\mathrm{B}$.

For case $\mathrm{B}$, which realizes the anti-twist with $\eta_{1}=\eta_{2}=1$, we find that the above conditions (3.29) are all satisfied provided that

$$
\text { Anti-twist : } \quad p_{1}, p_{2}>0, \quad p_{3}=n_{2}-n_{1}-p_{1}-p_{2}>0 .
$$

We can then show that, in particular, the central charge given in (3.28) is positive, $c>0$. This is the same result that was obtained in [5].

We also find that case $\mathrm{A}$, which realizes the twist with $\eta_{1}=-1, \eta_{2}=1$, is possible provided that

$$
\text { Twist : } \quad n_{2}>n_{1} \text { and two } p_{i} \text { are positive. }
$$

Notice here that since $p_{1}+p_{2}+p_{3}=-n_{1}-n_{2}$, given that two of the $p_{i}$ are positive, the third must then be negative. Moreover, these conditions imply that the central charge is positive, $c>0$. We also note that after formally setting $n_{1}=n_{2}$ in the expression for the central charge in case A with $\eta_{1}=-1, \eta_{2}=1$, we recover the expression for the central charge obtained in [21] for D3-branes wrapping a two-sphere with a topological twist. However, we emphasize that this is a formal statement, since e.g. from (3.25) we see that $y_{1}=y_{2}$ in this limit, giving a double root for the polynomial $P$, which is incompatible with having a compact spindle. Instead the solutions of [21] may be recovered from our local solutions by utilizing a scaling limit, as shown in appendix A.1. 
It is interesting to ask whether case A or B can be realized in minimal gauged supergravity. We will obtain solutions of minimal gauged supergravity after setting $p_{1}=p_{2}=$ $p_{3}=1 / 3\left(\eta_{1} n_{2}-\eta_{2} n_{1}\right)$, and we immediately find that only the anti-twist, case $\mathrm{B}$, is allowed, confirming what was already shown in [1]. It is also worth noting that if we formally set one of the fluxes to zero, say $p_{1}=0$, we will obtain a local solution that preserves twice as much supersymmetry. However, we can immediately see from the expression for the central charge, which vanishes in this limit, that this cannot make sense. Indeed, from (3.25) we see that we obtain a double root for the polynomial $P$ in this limit, and hence we will not obtain a compact spindle.

Another interesting limit to consider is when two charges are equal, for example $p_{1}=$ $p_{2} \neq 0$, with $p_{3}=-p_{1}-p_{2}+\left(\eta_{1} n_{2}-\eta_{2} n_{1}\right)$. For case $\mathrm{B}$, the anti-twist, we just need $p_{1}>0$ and $n_{2}-n_{1}>2 p_{1}$, as shown in [5]. We also find that case $\mathrm{A}$, the twist, is possible when we have $n_{2}>n_{1}$ and $p_{1}>0$. As mentioned earlier, these solutions can be uplifted on $M_{6}$ to give solutions of $D=11$ supergravity, as we discuss further in section 3.5.

\subsection{Killing spinors}

In this subsection we explicitly present the solutions to the Killing spinor equations (3.4). Some details of the derivation are presented in appendix C.

We begin by choosing the following orthonormal frame for the metric (3.6):

$$
\mathrm{e}^{a}=H^{1 / 6} \overline{\mathrm{e}}^{a}, \quad a=0,1,2, \quad \mathrm{e}^{3}=\frac{H^{1 / 6}}{2 P^{1 / 2}} \mathrm{~d} y, \quad \mathrm{e}^{4}=\frac{P^{1 / 2}}{H^{1 / 3}} \mathrm{~d} z,
$$

where $\overline{\mathrm{e}}^{a}$ is an orthonormal frame for the unit radius metric on $\mathrm{AdS}_{3}$. We use fivedimensional gamma matrices

$$
\Gamma_{a}=\beta_{a} \otimes \gamma_{3}, \quad \Gamma_{3}=1 \otimes \gamma_{1}, \quad \Gamma_{4}=1 \otimes \gamma_{2},
$$

where $\beta_{0}=\mathrm{i} \sigma^{2}, \beta_{1}=\sigma^{1}, \beta_{2}=\sigma^{3}$ are three-dimensional gamma matrices for $\mathrm{AdS}_{3}$ directions, while $\gamma_{1}=\sigma^{1}, \gamma_{2}=\sigma^{2}$ are two-dimensional gamma matrices for the spindle directions, with chirality matrix $\gamma_{3}=-\mathrm{i} \gamma_{1} \gamma_{2}=\sigma^{3}$.

We next introduce two-component $\mathrm{AdS}_{3}$ Killing spinors $\vartheta$ satisfying

$$
\nabla_{a} \vartheta=\frac{1}{2} \beta_{a} \vartheta
$$

whose two solutions $\vartheta_{+}^{(A)}, A=1,2$, are described explicitly in appendix B. We then find that the two $D=5$ Killing spinors $\epsilon^{(A)}, A=1,2$, solving (3.4) are

$$
\epsilon^{(A)}=\vartheta_{+}^{(A)} \otimes \zeta,
$$

where the two-component spinor $\zeta$ on the spindle is given by

$$
\zeta \equiv\left(\begin{array}{c}
\zeta_{+} \\
\zeta_{-}
\end{array}\right)=\frac{\mathrm{e}^{\mathrm{i} z}}{\sqrt{2} H^{1 / 6}}\left(\begin{array}{c}
{[\sqrt{H}-y]^{1 / 2}} \\
-[\sqrt{H}+y]^{1 / 2}
\end{array}\right)=H^{1 / 12} \mathrm{e}^{\mathrm{i} z}\left(\begin{array}{c}
\sin \frac{\alpha}{2} \\
-\cos \frac{\alpha}{2}
\end{array}\right)
$$


and the function $\alpha=\alpha(y)$ satisfies

$$
\sin \alpha=\frac{P^{1 / 2}}{H^{1 / 2}}, \quad \cos \alpha=\frac{y}{H^{1 / 2}} .
$$

In particular, the Killing spinors given by (3.35) satisfy the single projection condition

$$
M \epsilon^{(A)}=-\mathrm{i} \epsilon^{(A)}, \quad \text { where } \quad M \equiv \cos \alpha \Gamma_{34}+\mathrm{i} \sin \alpha \Gamma_{3},
$$

and one can verify that $M^{2}=-1$. We thus preserve half of the supersymmetries, corresponding to $d=2 \mathcal{N}=(0,2)$ supersymmetry. One also sees from (3.36) that the squared norm of the Killing spinor is

$$
\zeta^{\dagger} \zeta=H^{1 / 6}
$$

which is nowhere vanishing, and proportional to the square root of the warp factor, as expected from the general discussion in section 2.5. Moreover, on the spindle we have the Killing vector bilinear

$$
\frac{\mathrm{i}}{\zeta^{\dagger} \zeta}\left(\zeta^{\dagger} \gamma^{m} \gamma_{3} \zeta\right) \partial_{m}=\partial_{z}
$$

where here $x^{m}=(y, z)$ are coordinates on the spindle. On the $D=5$ spacetime we then deduce

$$
\left(\bar{\epsilon}^{(A)} \Gamma^{\mu} \epsilon^{(B)}\right) \partial_{\mu}=\left(\bar{\vartheta}_{+}^{(A)} \beta^{a} \vartheta_{+}^{(B)}\right) \partial_{a}+\mathrm{i}\left(\bar{\vartheta}_{+}^{(A)} \vartheta_{+}^{(B)}\right) \partial_{z},
$$

which reveals the way in which supersymmetry is being realized for the $\mathrm{AdS}_{3} \times \Sigma$ solutions. In particular, recalling the explicit Killing spinors on $\mathrm{AdS}_{3}$ (see appendix B) we conclude that, schematically, the last term in (3.41) implies that the azimuthal rotations on the spindle are acting as an R-symmetry for the $d=2, \mathcal{N}=(0,2)$ supersymmetry algebra. By contrast this term is absent in the supersymmetry algebra for $\mathrm{AdS}_{3} \times \Sigma_{g}$ solutions associated with a topological twist on the Riemann surface $\Sigma_{g}$.

Using the expression (3.36) for the Killing spinors, together with (3.10), one can immediately deduce

- Case A, Twist: $\zeta_{+}\left(y_{1}\right)=0$ and $\zeta_{+}\left(y_{2}\right)=0$, with $\zeta_{-} \neq 0$ everywhere.

- Case B, Anti-Twist: $\zeta_{-}\left(y_{1}\right)=0$ but $\zeta_{-}$is non-vanishing everywhere else, while $\zeta_{+}\left(y_{2}\right)=0$ but $\zeta_{+}$is non-vanishing everywhere else.

The corresponding R-symmetry flux in the two cases is

$$
Q^{R} \equiv \frac{1}{2 \pi} \int_{\Sigma} F^{R}=\frac{p_{1}+p_{2}+p_{3}}{n_{1} n_{2}}=\left\{\begin{aligned}
-\frac{n_{2}+n_{1}}{n_{1} n_{2}} & \text { Case A, Twist }, \\
\frac{n_{2}-n_{1}}{n_{1} n_{2}} & \text { Case B, Anti-Twist } .
\end{aligned}\right.
$$

Comparing to the general analysis in section 2.3, recall that to obtain the R-symmetry flux in $(2.38)$ we assumed $\zeta_{+}^{N}(0), \zeta_{+}^{S}(0) \neq 0$, while if instead $\zeta_{-}^{N}(0), \zeta_{-}^{S}(0) \neq 0$ one obtains the upper sign in (2.39). The latter then agrees with the R-symmetry flux in (3.42), where $n_{1}$ may be identified with either of $n_{S}$ or $n_{N}$ (and then respectively $n_{2}$ with either $n_{N}$ or 
$\left.n_{S}\right)$. On the other hand, for case $\mathrm{B}$ since $\zeta_{+}$is non-vanishing at $y_{1}$, we identify $y_{1}=y_{N}$, $y_{2}=y_{S}$, and the R-symmetry flux in (3.42) agrees with the anti-twist in equation (2.38).

Note that in the gauge we are using for $A^{(I)}$, the R-symmetry gauge field is not regular at either pole located at $y=y_{1}$ or $y=y_{2}$. However, using (3.20) we may obtain a smooth gauge field at $y=y_{1}$ via the gauge transformation $A^{R} \rightarrow A_{(0)}^{R}=A^{R}-2 \mathrm{~d} z+\frac{\eta_{1}}{n_{1}} \mathrm{~d} \varphi$. Here we have introduced $\varphi=\frac{2 \pi}{\Delta z} z$, with $\Delta \varphi=2 \pi$, and the subscript on $A_{(0)}^{R}$ indicates that this gauge field is smooth at the pole, as in the notation and discussion in section 2 . In particular the shift by $\frac{\eta_{1}}{n_{1}} \mathrm{~d} \varphi$ is equivalent to an $\mathrm{SL}(2, \mathbb{Z})$ transformation in the covering space. This gauge transformation will act on the spinor, which is only charged under the R-symmetry, and replace the $\mathrm{e}^{\mathrm{i} z}$ phase in (3.36) with $\mathrm{e}^{\frac{\mathrm{i} \eta_{1} \varphi}{2 n_{1}}}$. Noting that the frame we are using is the analogue of the hatted frame in section 2 (which is the frame that is not regular at $y=y_{1}$ ), we see that this behaviour is in agreement with (2.26). Recall here that for the twist case $\mathrm{A}, \zeta_{+}\left(y_{1}\right)=0$ and $\eta_{1}=-1$, while for the anti-twist case $\mathrm{B}, \zeta_{-}\left(y_{1}\right)=0$ and $\eta_{1}=+1$. It then follows from (3.36) that in the locally Euclidean frame and in the new gauge, for the twist case $\mathrm{A}$ we have $\zeta_{+} \sim \mathrm{e}^{-\frac{\mathrm{i} \varphi}{n_{1}}} \rho_{1}$, where $\rho_{1}$ is the radial coordinate at $y=y_{1}$, while for the anti-twist case $\mathrm{B}$ we instead have $\zeta_{-} \sim \mathrm{e}^{+\frac{\mathrm{i} \varphi}{n_{1}}} \rho_{1}$. In both cases we have a simple holomorphic zero of the spinor to leading order at the pole $\rho_{1}=0$. Similar comments apply, mutatis mutandis, to the behaviour of the spinors at the other pole located at $y=y_{2}$.

Finally, as already noted, the spinor (3.36) is charged under azimuthal rotations of the spindle, due to the phase $\mathrm{e}^{\mathrm{i} z}$. We consider the following two-parameter family of gauge transformations on the gauge fields

$$
\tilde{A}^{(I)} \equiv A^{(I)}+\mathbf{s}^{I} \mathrm{~d} z=\rho_{I}(y) \mathrm{d} \varphi,
$$

where the $\mathbf{s}^{I}$ are constants satisfying the constraint

$$
s^{1}+s^{2}+s^{3}=-2 .
$$

The latter ensures that the R-symmetry gauge field transforms to $\tilde{A}^{R}=A^{R}-2 \mathrm{~d} z$, which cancels the phase $\mathrm{e}^{\mathrm{i} z}$ in the spinor as the spinor has charge $\frac{1}{2}$ under $A^{R}$. In (3.43) we have introduced the functions

$$
\rho_{I}(y) \equiv\left(\frac{y}{h_{I}}+\mathbf{s}^{I}\right) \frac{\Delta z}{2 \pi},
$$

which will play a role in the field theory analysis in the next subsection (where we will also see that the gauge choice of all $\mathbf{s}^{I}$ equal, namely $\mathbf{s}^{I}=-2 / 3$, has a notable property). From (3.43) one can check that

$$
\left.\tilde{A}^{R}\right|_{y=y_{1}}=-\frac{\eta_{1}}{n_{1}} \mathrm{~d} \varphi,\left.\quad \tilde{A}^{R}\right|_{y=y_{2}}=-\frac{\eta_{2}}{n_{2}} \mathrm{~d} \varphi
$$

This agrees with the general conditions for a local gauge in which the spinor is uncharged with respect to azimuthal rotations that we derived earlier (2.49), where in particular recall that our twist case A is a charge conjugate of the twist considered in section 2. 


\subsection{Comparison with field theory}

As in [5], there is a natural field theory interpretation ${ }^{16}$ of the solutions we have constructed. One begins with $d=4, \mathcal{N}=4 \mathrm{SYM}$ theory, which is dual to the $\mathrm{AdS}_{5} \times S^{5}$ vacuum of the $D=5$, STU U $(1)^{3}$ gauged supergravity theory. One then compactifies this field theory on the spindle $\Sigma$, together with magnetic fluxes $Q_{I}$ (3.14) for the three Abelian gauge fields. The existence of the $\mathrm{AdS}_{3}$ supergravity solutions suggests that this compactified theory flows to a $d=2,(0,2)$ SCFT in the IR. There is an important distinction here between the twist and anti-twist solutions, that we discuss further in section 6 .

Following $[1,5]$, we consider the anomaly polynomial of $\mathcal{N}=4 \mathrm{SYM}$, with background gauge fields fluxes $F^{(I)}$ for the $\mathrm{U}(1)^{3} \subset \mathrm{SO}(6)$ Abelian global symmetry group. In the large $N$ limit this reads

$$
\mathcal{A}_{4 \mathrm{~d}}=c_{1}\left(F^{(1)}\right) c_{1}\left(F^{(2)}\right) c_{1}\left(F^{(3)}\right) \frac{N^{2}}{2} .
$$

Here $c_{1}\left(F^{(I)}\right)$ denote the first Chern classes of the $\mathrm{U}(1)$ bundles with gauge field curvatures $F^{(I)}$. Recalling the expression (3.43) for the gauge field, we next introduce connection oneforms

$$
\mathscr{A}^{(I)}=\rho_{I}(y)\left(\mathrm{d} \varphi+\mathcal{A}_{\mathcal{J}}\right)
$$

with curvature

$$
\mathscr{F}^{(I)}=\mathrm{d} \mathscr{A}^{(I)}=\rho_{I}^{\prime}(y) \mathrm{d} y \wedge\left(\mathrm{d} \varphi+\mathcal{A}_{\mathcal{J}}\right)+\rho_{I}(y) \mathcal{F}_{\mathcal{J}}
$$

where $\mathcal{F}_{\mathcal{J}}=\mathrm{d} \mathcal{A}_{\mathcal{J}}$. Here $\mathcal{A}_{\mathcal{J}}$ gauges the azimuthal symmetry of the spindle. In particular, this means that setting $\mathcal{A}_{\mathcal{J}}=0$ the $\mathscr{A}^{(I)}$ reduce to the gauge fields $\tilde{A}^{(I)}$ in (3.43) i.e. in a gauge where the Killing spinor is uncharged under azimuthal rotations of the spindle generated by $\partial_{\varphi}$. This implies the flux conditions

$$
\frac{1}{2 \pi} \int_{\Sigma} \mathscr{F}^{(I)}=\rho_{I}\left(y_{2}\right)-\rho_{I}\left(y_{1}\right)=\frac{p_{I}}{n_{2} n_{1}} \quad(I=1,2,3)
$$

together with the constraints (3.46) at the poles:

$$
\sum_{I=1}^{3} \rho_{I}\left(y_{1}\right)=-\frac{\eta_{1}}{n_{1}}, \quad \sum_{I=1}^{3} \rho_{I}\left(y_{2}\right)=-\frac{\eta_{2}}{n_{2}} .
$$

The general solution to the constraints $(3.50)$, (3.51) for $\rho_{I}\left(y_{i}\right)$, with $p_{3}$ determined by $(3.21)$, is

$$
\begin{array}{rlrl}
\rho_{1}\left(y_{1}\right) & =\alpha_{1}, \quad \rho_{2}\left(y_{1}\right)=\alpha_{2}, & \rho_{3}\left(y_{1}\right)=-\frac{\eta_{1}}{n_{1}}-\alpha_{1}-\alpha_{2}, \\
\rho_{1}\left(y_{2}\right)=\frac{p_{1}}{n_{1} n_{2}}+\alpha_{1}, & \rho_{2}\left(y_{2}\right)=\frac{p_{2}}{n_{1} n_{2}}+\alpha_{2}, \\
\rho_{3}\left(y_{2}\right)=-\frac{\eta_{2}}{n_{2}}-\frac{p_{1}}{n_{1} n_{2}}-\frac{p_{2}}{n_{2} n_{1}}-\alpha_{1}-\alpha_{2}, & &
\end{array}
$$

\footnotetext{
${ }^{16}$ See [1] for a more general interpretation for the solutions of minimal gauged supergravity and also section 3.5 for an M5-brane interpretation of a sub-class.
} 
where $\alpha_{1}, \alpha_{2}$ are a priori arbitrary. Notice that the $\mathrm{AdS}_{3}$ supergravity solution gave explicit functions (3.45) for $\rho_{I}(y)$, although these are determined up to a choice of $\mathbf{s}^{I}$ satisfying the constraint (3.44). This is equivalent to the freedom of choosing $\alpha_{1}, \alpha_{2}$ above. However, we shall see momentarily that the central charge of the theory is independent of $\alpha_{1}, \alpha_{2}$.

The curvature form $\mathscr{F}^{(I)}$ defines a corresponding first Chern class $c_{1}\left(\mathcal{L}_{I}\right)=\left[\mathscr{F}^{(I)} / 2 \pi\right]$, and similarly we define $c_{1}(\mathcal{J})=\left[\mathcal{F}_{\mathcal{J}} / 2 \pi\right]$. We then write

$$
c_{1}\left(F^{(I)}\right)=\Delta_{I} c_{1}\left(R_{2 \mathrm{~d}}\right)+c_{1}\left(\mathcal{L}_{I}\right),
$$

with the trial R-charges $\Delta_{I}$ constrained to satisfy $\Delta_{1}+\Delta_{2}+\Delta_{3}=2$, so that the superpotential of $\mathcal{N}=4 \mathrm{SYM}$ has R-charge 2 , in $\mathcal{N}=1$ language. The $d=2$ anomaly polynomial is then obtained by integrating $\mathcal{A}_{4 \mathrm{~d}}$ over $\Sigma$ :

$$
\begin{aligned}
\mathcal{A}_{2 \mathrm{~d}}=\int_{\Sigma} \mathcal{A}_{4 \mathrm{~d}}= & {\left[\left(\Delta_{1} \Delta_{2} I_{3}+\Delta_{2} \Delta_{3} I_{1}+\Delta_{3} \Delta_{1} I_{2}\right) c_{1}\left(R_{2 \mathrm{~d}}\right)^{2}\right.} \\
& \left.+\left(\Delta_{1} I_{4}+\Delta_{2} I_{5}+\Delta_{3} I_{6}\right) c_{1}\left(R_{2 \mathrm{~d}}\right) c_{1}(\mathcal{J})+I_{7} c_{1}(\mathcal{J})^{2}\right] \frac{N^{2}}{2} .
\end{aligned}
$$

Here

$$
\begin{array}{lll}
I_{I}=\rho_{I}\left(y_{2}\right)-\rho_{I}\left(y_{1}\right) \equiv\left[\rho_{I}\right]_{y_{1}}^{y_{2}}, & I_{4}=\left[\rho_{2} \rho_{3}\right]_{y_{1}}^{y_{2}}, & I_{5}=\left[\rho_{3} \rho_{1}\right]_{y_{1}}^{y_{2}}, \\
I_{6}=\left[\rho_{1} \rho_{2}\right]_{y_{1}}^{y_{2}}, & I_{7}=\left[\rho_{1} \rho_{2} \rho_{3}\right]_{y_{1}}^{y_{2}} . &
\end{array}
$$

The $d=2$ superconformal R-symmetry extremizes the trial function

$$
c_{\text {trial }}=3 \operatorname{tr} \gamma_{3} R_{\text {trial }}^{2}
$$

where

$$
R_{\text {trial }}=R_{2 \mathrm{~d}}+\varepsilon \mathcal{J}
$$

From (3.54) we find

$$
\begin{aligned}
c_{\text {trial }}=3[ & \left(\Delta_{1} \Delta_{2} I_{3}+\Delta_{2} \Delta_{3} I_{1}+\Delta_{3} \Delta_{1} I_{2}\right)+\left(\Delta_{1} I_{4}+\Delta_{2} I_{5}+\Delta_{3} I_{6}\right) \varepsilon \\
& \left.+I_{7} \varepsilon^{2}\right] N^{2} .
\end{aligned}
$$

Extremizing over $\Delta_{1}, \Delta_{2}$ and $\varepsilon$, we obtain the extremal values

$$
\begin{aligned}
\Delta_{1}^{\star} & =-2 \frac{p_{1}}{s}\left(\eta_{2} n_{1}+p_{1}\right)-\varepsilon^{*} \alpha_{1}, & \Delta_{2}^{\star} & =-2 \frac{p_{2}}{s}\left(\eta_{2} n_{1}+p_{2}\right)-\varepsilon^{*} \alpha_{2}, \\
\varepsilon^{\star} & =2 \frac{n_{2} n_{1}}{s}\left(\eta_{1} n_{2}+\eta_{2} n_{1}\right), & c^{\star} & =\frac{6 p_{1} p_{2} p_{3}}{n_{2} n_{1} s} N^{2},
\end{aligned}
$$

where note that $c^{\star}$ agrees with the general supergravity result (3.28). Notice that neither of the critical values $\varepsilon^{\star}$ nor $c^{\star}$ depend on the parameters $\alpha_{1}, \alpha_{2}$, but the critical R-charges $\Delta_{I}^{\star}$ do. This is a gauge freedom, and in fact the parameters $\alpha_{1}, \alpha_{2}$ may be removed from $c_{\text {trial }}$ via the change of variable $\Delta_{I} \rightarrow \Delta_{I}-\varepsilon \alpha_{I}, I=1,2\left(\right.$ where $\left.\Delta_{3}=2-\Delta_{1}-\Delta_{2}\right)$. We also note that by choosing $\alpha_{1}, \alpha_{2}$ with $\mathbf{s}^{I}=-2 / 3$ in (3.45) we find the extremal values $\Delta_{1}^{\star}=\Delta_{2}^{\star}=\Delta_{3}^{\star}=2 / 3$, the notable property we alluded to earlier (just below (3.45)). 
In the limit $n_{1}=n_{2}=1$, when the spindle reduces to a two-sphere, we should be able to formally compare our results with (3.12) of reference [21], where their $\eta_{\Sigma}=2, d_{G}=N^{2}$, $\mathfrak{g}=0$ and $\kappa=1$. We find

$$
c_{B B}=\frac{24 a_{1} a_{2}\left(1+a_{1}+a_{2}\right)}{1+4\left(a_{1}^{2}+a_{2}^{2}+a_{1} a_{2}+a_{1}+a_{2}\right)} N^{2} .
$$

This indeed agrees with the $n_{1}=n_{2}=1$ limit of $c^{\star}$ in the twist case A, identifying $a_{i}=p_{i} / 2$. Notice the configurations of [21] are a topological twist for the two-sphere, and arise here as a limit of our more general twist case A spindle configurations. Note, however, that the supergravity solutions of [21] do not arise as a limit of the solutions for the spindle, but instead can be obtained from the local solutions (3.6) after taking a certain scaling limit, as discussed in appendix A.1.

\subsection{M5-branes}

As noted above there is an alternative field theory interpretation of the solutions of $D=5$ gauged supergravity that we have constructed in the special case that two of the magnetic fluxes are equal. Start with a $d=4, \mathcal{N}=2$ field theory that is dual to an $\operatorname{AdS}_{5} \times M_{6}$ solution of $D=11$ supergravity of the type discussed in $[24,26]$. One can then compactify on the spindle $\Sigma$, together with magnetic fluxes $Q_{I}$ (3.14) for the three Abelian gauge fields, with two equal. The existence of our corresponding $\mathrm{AdS}_{3}$ supergravity solutions, uplifted to $D=11$ using the results of [25], indicates that this compactified theory flows to a $d=2, \mathcal{N}=(0,2) \mathrm{SCFT}$ in the IR.

Focusing, for simplicity, on the explicit case of the $\mathrm{AdS}_{5} \times M_{6}$ solution given in [11], associated with $N$ M5-branes wrapping a Riemann surface of genus $g$, and setting $p_{1}=p_{2}$ for definiteness, it was shown in [5] for the anti-twist solutions that the central charge of the $d=2 \mathrm{SCFT}$ can be written in the form

$$
c=\frac{8 p_{1}^{2} p_{3}}{n_{1} n_{2} s}(g-1) N^{3},
$$

where

$$
\text { Anti-Twist : } \quad p_{1}=p_{2}>0, \quad p_{3}=n_{2}-n_{1}-2 p_{1}>0 .
$$

In fact the analysis in [5] can immediately be adapted to the twist case and we find a central charge as in (3.61), but now with $p_{1}=p_{2}>0, n_{2}>n_{1}$ and

$$
\text { Twist : } \quad p_{1}=p_{2}>0, \quad n_{2}>n_{1} \Rightarrow p_{3}=-n_{1}-n_{2}-2 p_{1}<0 .
$$

Furthermore, the field theory analysis of [5] using the large $N$ limit of the M5-brane anomaly polynomial which was used to recover the gravity central charge for the anti-twist case, can also be adapted for the case of the twist and again we find exact agreement.

All of these configurations preserve $\mathcal{N}=(0,2)$ supersymmetry. Configurations with $\mathcal{N}=(0,4)$ or $\mathcal{N}=(2,2)$ supersymmetry are linked with setting $p_{1}=p_{2}=0$ or $p_{3}=0$, associated, in the twist class, with M5-branes wrapping a product of $\Sigma \times \Sigma_{g}$ in a Calabi-Yau three fold or a product of two Calabi-Yau two-folds, respectively. However, we do not have 
supergravity solutions with compact spindles for either of these values as one can conclude from the vanishing of the central charge.

It is also interesting to point out that if we formally set $n_{1}=n_{2}=1$ in the twist class we find

$$
c=\frac{8 p_{1}^{2}}{1+3 p_{1}}(g-1) N^{3},
$$

and this should be compared with the central charge of the $S^{2} \times \Sigma_{g} D=11$ supergravity solutions, first found in section 3.4 of [25] and subsequently discussed in [21]. In the notation of the latter reference one should set $\kappa_{1}=1, \kappa_{2}=-1, \eta_{1}=2, \eta_{2}=2(g-1), z_{1}=2 a_{1}+1$ and $z_{2}=2 a_{2}-1$, and then set $a_{2}=0$ (i.e. $z_{2}=-1$ ). One then finds the central charge

$$
c_{B B}=\frac{8 a_{1}^{2}}{1+3 a_{1}}(g-1) N^{3} .
$$

This agrees with (3.64), identifying $a_{1}=p_{1}$.

\section{M2-branes}

In this section we re-examine M2-branes wrapping spindles, generalizing earlier work in [3, $7,8]$. The analysis is more involved than for the D3-brane case studied in section 3, and our treatment will correspondingly be less comprehensive. However, a key point is that there again exist new twist solutions in addition to the previously known anti-twist solutions, with the twist solutions naturally interpreted as near-horizon limits of M2-branes wrapping a holomorphically embedded spindle inside a Calabi-Yau five-fold.

\subsection{The $D=4$ gauged supergravity model}

The $D=4, \mathrm{U}(1)^{4}$ gauged supergravity model that we shall consider has a Lagrangian given by (see e.g. [29])

$$
\mathcal{L}=\sqrt{-g}\left[R-\mathcal{V}-\frac{1}{2} \sum_{I=1}^{4}\left(X^{(I)}\right)^{-2}\left(\partial X^{(I)}\right)^{2}-\frac{1}{4} \sum_{I=1}^{4}\left(X^{(I)}\right)^{-2}\left(F^{(I)}\right)^{2}\right] .
$$

Here $A^{(I)}, I=1, \ldots, 4$, are four $\mathrm{U}(1)$ gauge fields, with field strengths $F^{(I)}=\mathrm{d} A^{(I)}$. The four scalar fields $X^{(I)}>0$, which satisfy the constraint $X^{(1)} X^{(2)} X^{(3)} X^{(4)}=1$, can be written in terms of three canonically normalized scalars $\varphi_{1,2,3}$ via

$$
\begin{array}{ll}
X^{(1)}=\mathrm{e}^{+\frac{\varphi_{1}}{2}+\frac{\varphi_{2}}{2}+\frac{\varphi_{3}}{2}}, & X^{(2)}=\mathrm{e}^{+\frac{\varphi_{1}}{2}-\frac{\varphi_{2}}{2}-\frac{\varphi_{3}}{2}}, \\
X^{(3)}=\mathrm{e}^{-\frac{\varphi_{1}}{2}+\frac{\varphi_{2}}{2}-\frac{\varphi_{3}}{2}}, & X^{(4)}=\mathrm{e}^{-\frac{\varphi_{1}}{2}-\frac{\varphi_{2}}{2}+\frac{\varphi_{3}}{2}},
\end{array}
$$

in terms of which the potential can be written

$$
\mathcal{V}=-2 \sum_{i=1}^{3} \cosh \left(\varphi_{i}\right)=-\sum_{I<J} X^{(I)} X^{(J)} .
$$

This model is a truncation of an $\mathcal{N}=2$ gauged supergravity theory coupled to three vector multiplets, with three axion fields set to zero. A solution of our model with $F^{(I)} \wedge$ 
$F^{(J)}=0$ will be a solution of the $\mathcal{N}=2$ theory, and can be uplifted to $D=11$ supergravity on an $S^{7}$ using the results of [23]. In particular, the vacuum $\mathrm{AdS}_{4}$ solution, which has unit radius, uplifts to the $\mathrm{AdS}_{4} \times S^{7}$ solution, dual to $d=3 \mathrm{ABJM}$ theory.

A solution will preserve supersymmetry provided that we can find solutions to the Killing spinor equations

$$
\begin{aligned}
0 & =\left[\nabla_{\mu}-\frac{\mathrm{i}}{4} \sum_{I=1}^{4} A^{(I)}+\frac{1}{8} \sum_{I=1}^{4} X^{(I)} \Gamma_{\mu}+\frac{\mathrm{i}}{16} \sum_{I=1}^{4}\left(X^{(I)}\right)^{-1} F_{\nu \rho}^{(I)} \Gamma^{\nu \rho} \Gamma_{\mu}\right] \epsilon, \\
0 & =\left[\not \partial \varphi_{i}-\sum_{I=1}^{4} \partial_{\varphi_{i}} X^{(I)}+\frac{\mathrm{i}}{2} \sum_{I=1}^{4} \partial_{\varphi_{i}}\left(X^{(I)}\right)^{-1} F_{\mu \nu}^{(I)} \Gamma^{\mu \nu}\right] \epsilon
\end{aligned}
$$

where $\Gamma^{\mu}$ are $D=4$ Gamma matrices, $\nabla=\mathrm{d}+\frac{1}{4} \omega^{a b} \Gamma_{a b}$, with $\omega^{a b}$ the Levi-Civita connection, and $\epsilon$ is a Dirac spinor. We can define the R-symmetry gauge field via

$$
A^{R} \equiv \frac{1}{2} \sum_{I=1}^{4} A^{(I)},
$$

which is then normalized as in section 2 .

\subsection{Supergravity solution and global analysis}

We consider an $\mathrm{AdS}_{2} \times \Sigma$ solution of this theory with four independent magnetic charges, which was introduced in [10] as a solution of $D=11$ supergravity (after uplifting on $S^{7}$ ), and revisited in $[7,8]$ from a purely four-dimensional point of view. The metric, gauge fields and scalars of this solution read

$$
\begin{aligned}
& \mathrm{d} s_{4}^{2}=H^{1 / 2}\left[\frac{1}{4} \mathrm{~d} s_{\mathrm{AdS} S_{2}}^{2}+\frac{1}{P} \mathrm{~d} y^{2}+\frac{P}{4 H} \mathrm{~d} z^{2}\right]=H^{1 / 2}\left[\frac{1}{4} \mathrm{~d} s_{\mathrm{AdS}_{2}}^{2}+\mathrm{d} s_{\Sigma}^{2}\right], \\
& A^{(I)}=\frac{y}{h_{I}} \mathrm{~d} z, \quad X^{(I)}=\left(\frac{h_{1} h_{2} h_{3} h_{4}}{h_{I}^{4}}\right)^{1 / 4},
\end{aligned}
$$

where $\mathrm{d} s_{\mathrm{AdS}_{2}}^{2}$ is a unit radius metric and $h_{I}, H$ and $P$ are all functions of $y$ given by

$$
h_{I}=y+q_{I}, \quad H=h_{1} h_{2} h_{3} h_{4}, \quad P=H-4 y^{2},
$$

and $q_{I}, I=1,2,3,4$ are constants. Note that (4.6) is a solution of the equations of motion only when all $h_{I}$ have the same sign, either positive or negative. Note that we can write the field strength as

$$
\left(X^{(I)}\right)^{-2} F^{(I)}=\frac{2 q_{I}}{H^{1 / 2}} \widetilde{\operatorname{vol}}_{\Sigma},
$$

where $\widetilde{\operatorname{vol}}(\Sigma)$ is the volume form associated to the conformally rescaled spindle metric $\widetilde{\mathrm{d} s^{2}}{ }_{\Sigma}=H^{1 / 2} \mathrm{~d} s_{\Sigma}^{2}$.

We want to choose the parameters so that $(y, z)$ parametrize a compact spindle, with positive warp factor $H>0$ as well as $X^{(I)}>0$. It is technically difficult to be as explicit as we were for the case of the solutions of $D=5$ gauged supergravity but we can still make significant progress. We assume that the quartic polynomial $P$ has four distinct real roots 
$y_{0}<y_{1}<y_{2}<y_{3}$, and we take $y \in\left[y_{1}, y_{2}\right]$ with $P \geq 0$ in this range. Expanding near the boundaries of this interval and setting $\varrho_{i}=2\left|y-y_{i}\right|^{1 / 2}$ for $i=1,2$ we find

$$
\mathrm{d} s_{\Sigma}^{2}=\frac{1}{\left|P^{\prime}\left(y_{i}\right)\right|}\left(\mathrm{d} \varrho_{i}^{2}+\kappa_{i}^{2} \varrho_{i}^{2} \mathrm{~d} z^{2}\right), \quad \text { where } \quad \kappa_{i}=\left|\frac{P^{\prime}\left(y_{i}\right)}{8 y_{i}}\right|, \quad i=1,2 .
$$

With $P^{\prime}\left(y_{1}\right)>0$ and $P^{\prime}\left(y_{2}\right)<0$, we again distinguish three cases corresponding to the three possible signs for $y_{i}(i=1,2)$, by setting

$$
\eta_{1} y_{1}<0, \quad \eta_{2} y_{2}>0
$$

with

$$
\begin{array}{ll}
\text { Case A : } & \left(\eta_{1}, \eta_{2}\right)=(-1,+1), \\
\text { Case B : } & \left(\eta_{1}, \eta_{2}\right)=(+1,+1), \\
\text { Case C : } & \left(\eta_{1}, \eta_{2}\right)=(+1,-1) .
\end{array}
$$

Then, for all three cases we can write

$$
\kappa_{i}=-\frac{\eta_{i} P^{\prime}\left(y_{i}\right)}{8 y_{i}}
$$

and $\Sigma$ will be a spindle provided we impose

$$
\Delta z=\frac{2 \pi}{\kappa_{1} n_{1}}=\frac{2 \pi}{\kappa_{2} n_{2}},
$$

where $n_{1}, n_{2} \in \mathbb{N}$ with $\operatorname{hcf}\left(n_{1}, n_{2}\right)=1$. As in the previous section we then can immediately determine the Euler number of the spindle, finding $\chi(\Sigma)=\left(n_{1}+n_{2}\right) /\left(n_{1} n_{2}\right)$ as expected.

We now demand that the four gauge field fluxes on the spindle are suitably quantized. Observing from (4.4) that the $D=4$ spinors carry charge $\frac{1}{4}$ with respect to each of the gauge fields, we demand

$$
\begin{aligned}
Q_{I} & =\frac{1}{2 \pi} \int_{\Sigma} F^{(I)}=\left(\frac{y_{2}}{h_{I}\left(y_{2}\right)}-\frac{y_{1}}{h_{I}\left(y_{1}\right)}\right) \frac{\Delta z}{2 \pi} \\
& \equiv \frac{2 p_{I}}{n_{1} n_{2}}, \quad p_{I} \in \mathbb{Z} .
\end{aligned}
$$

We can now compute the total R-symmetry flux defined as $Q^{R} \equiv \frac{1}{2}\left(Q_{1}+Q_{2}+Q_{3}+Q_{4}\right)$, which is normalized as in section 2 . To that end, one can use the identities

$$
\begin{aligned}
P^{\prime} & =h_{1} h_{2} h_{3}+h_{1} h_{2} h_{4}+h_{2} h_{3} h_{4}+h_{1} h_{3} h_{4}-8 y, \\
y_{i}^{2} & =\frac{1}{4} h_{1}\left(y_{i}\right) h_{2}\left(y_{i}\right) h_{3}\left(y_{i}\right) h_{4}\left(y_{i}\right), \quad(i=1,2),
\end{aligned}
$$

in (4.12), which allows us to rewrite (4.13) as

$$
\frac{\Delta z}{2 \pi}\left[1-\frac{y_{i}}{2} \sum_{I=1}^{4} \frac{1}{h_{I}\left(y_{i}\right)}\right]=\frac{\eta_{i}}{n_{i}}, \quad(\text { no sum on } i) .
$$




\begin{tabular}{|c|c|c|c|c|c|c|}
\hline Case & $q_{1}$ & $q_{2}$ & $q_{3}$ & $q_{4}$ & $y_{1}$ & $y_{2}$ \\
\hline $\mathrm{A}$ & -0.01 & 0.44 & 0.72 & 0.97 & 0.01 & 0.11 \\
\hline $\mathrm{B}$ & 0.03 & 0.11 & 0.67 & 0.95 & -0.01 & 0.05 \\
\hline $\mathrm{C}$ & 0.01 & -0.44 & -0.72 & -0.97 & -0.11 & -0.01 \\
\hline
\end{tabular}

Table 1. Values of the parameters $q_{I}$, and corresponding roots $y_{1,2}$, for which the three cases are realized.

One can now immediately prove that the R-symmetry flux is given by

$$
Q^{R}=\frac{\eta_{1} n_{2}-\eta_{2} n_{1}}{n_{1} n_{2}} \quad \Leftrightarrow \quad p_{1}+p_{2}+p_{3}+p_{4}=\eta_{1} n_{2}-\eta_{2} n_{1} .
$$

Case B is the anti-twist; this is the only case realized when all four charges are equal, hence giving a solution of minimal gauged supergravity [3]. Cases $\mathrm{A}$ and $\mathrm{C}$ correspond to twist solutions, which in fact can only be realized in the model with four distinct charges, as discussed below.

As mentioned earlier, we could not solve explicitly the conditions for regularity in general, so one might wonder whether there exist choices of the parameters $q_{I}$ for which the three cases A, B, C are realized in practice. To end this section, in table 1 we give some possible values of the $q_{I}$ which lead to regular spindle solutions in the three cases. In particular, let us observe that analysing the roots numerically it seems that case A can only be realized when all $h_{I}>0$, case B admits both all $h_{I}>0$ and all $h_{I}<0$, while case $\mathrm{C}$ is only possible if all $h_{I}<0$. Note that the map

$$
y \rightarrow-y, \quad q_{I} \rightarrow-q_{I},
$$

is a symmetry of the solution, and it maps case A solutions to case $\mathrm{C}$ solutions, while case $\mathrm{B}$ is mapped to itself.

Let us make a comment on the case with pairwise equal charges. For concreteness, set $q_{1}=q_{2}$ and $q_{3}=q_{4}$, so that $h_{1}=h_{2}$ and $h_{3}=h_{4}$. One can wonder whether twist solutions (for either case A or case $\mathrm{C}$ ) exist in this case, but it turns out that the two middle roots of $P$ have the same sign only when $h_{1} h_{3}<0$, which violates the requirement that all $h_{I}$ have the same sign. This rules out twist solutions, leading to the anti-twist solutions discussed in $[7,8]$.

As conjectured in appendix $\mathrm{C}$ of [7], the $\mathrm{AdS}_{2} \times \Sigma$ solutions with four magnetic charges discussed in this section should arise as the near-horizon limit of certain accelerating black holes in $\mathrm{AdS}_{4}$, which are the electro-magnetic dual of the black holes found in [30]. Their entropy can be computed directly from the near-horizon solution, and reads

$$
S_{B H}=\frac{A_{\text {horizon }}}{4 G_{(4)}}=\frac{y_{2}-y_{1}}{8 G_{(4)}} \Delta z,
$$

where $G_{(4)}$ is the four-dimensional Newton constant. Following the conventions of [7], this can be expressed in terms of the number $N$ of M2-branes as

$$
\frac{1}{G_{(4)}}=\frac{2 \sqrt{2}}{3} N^{3 / 2} \text {. }
$$




\subsection{Killing spinors}

We begin by choosing the following orthonormal frame for the metric (4.6):

$$
\mathrm{e}^{a}=\frac{1}{2} H^{1 / 4} \overline{\mathrm{e}}^{a}, \quad a=0,1, \quad \mathrm{e}^{2}=\frac{H^{1 / 4}}{P^{1 / 2}} \mathrm{~d} y, \quad \mathrm{e}^{3}=\frac{P^{1 / 2}}{2 H^{1 / 4}} \mathrm{~d} z,
$$

where $\overline{\mathrm{e}}^{a}$ is an orthonormal frame for the unit radius metric on $\mathrm{AdS}_{2}$. We use $D=4$ gamma matrices

$$
\Gamma_{a}=\beta_{a} \otimes \gamma_{3}, \quad \Gamma_{2}=1 \otimes \gamma_{1}, \quad \Gamma_{3}=1 \otimes \gamma_{2},
$$

where $\beta_{0}=\mathrm{i} \sigma^{2}, \beta_{1}=\sigma^{1}$ are two-dimensional gamma matrices for the $\mathrm{AdS}_{2}$ directions, while $\gamma_{1}=\sigma^{1}, \gamma_{2}=\sigma^{2}$ are two-dimensional gamma matrices for the spindle directions, with chirality matrix $\gamma_{3}=-\mathrm{i} \gamma_{1} \gamma_{2}=\sigma^{3}$.

We next introduce two-component $\mathrm{AdS}_{2}$ Killing spinors $\vartheta$ satisfying

$$
\nabla_{a} \vartheta=\frac{\mathrm{k}}{2} \gamma_{a} \vartheta .
$$

As in appendix B, we have two solutions $\vartheta_{+}^{1,2}$ when $\mathrm{k}=+1$ and two solutions $\vartheta_{-}^{1,2}$ when $\mathrm{k}=-1$, which in particular are Majorana spinors. We then find that the $D=4$ Killing spinors $\epsilon^{(A)}, A=1,2$, solving (4.4) are different according to whether the functions $h_{I}$ are all positive or all negative. In particular, we find

$$
\epsilon^{(A)}= \begin{cases}\vartheta_{+}^{(A)} \otimes \zeta & \text { all } h_{I}>0 \\ \vartheta_{-}^{(A)} \otimes \tilde{\zeta} & \text { all } h_{I}<0\end{cases}
$$

where the spinors $\zeta$ and $\tilde{\zeta}$ on the spindle are given by

$$
\zeta \equiv\left(\begin{array}{c}
\zeta_{+} \\
\zeta_{-}
\end{array}\right)=\frac{\mathrm{e}^{\frac{\mathrm{i} z}{2}}}{\sqrt{2} H^{1 / 8}}\left(\begin{array}{c}
{[\sqrt{H}-2 y]^{1 / 2}} \\
-[\sqrt{H}+2 y]^{1 / 2}
\end{array}\right)=\mathrm{e}^{\mathrm{i} z} H^{1 / 8}\left(\begin{array}{c}
\sin \frac{\alpha}{2} \\
-\cos \frac{\alpha}{2}
\end{array}\right),
$$

and

$$
\tilde{\zeta} \equiv\left(\begin{array}{l}
\tilde{\zeta}_{+} \\
\tilde{\zeta}_{-}
\end{array}\right)=\frac{\mathrm{e}^{\frac{\mathrm{i} z}{2}}}{\sqrt{2} H^{1 / 8}}\left(\begin{array}{l}
{[\sqrt{H}-2 y]^{1 / 2}} \\
{[\sqrt{H}+2 y]^{1 / 2}}
\end{array}\right)=\mathrm{e}^{\frac{\mathrm{i} z}{2}} H^{1 / 8}\left(\begin{array}{c}
\sin \frac{\alpha}{2} \\
\cos \frac{\alpha}{2}
\end{array}\right),
$$

respectively, where the function $\alpha=\alpha(y)$ satisfies

$$
\sin \alpha=\frac{P^{1 / 2}}{H^{1 / 2}}, \quad \cos \alpha=\frac{2 y}{H^{1 / 2}} .
$$

In particular the spinors $\epsilon^{(A)}$ given by (4.24) satisfy

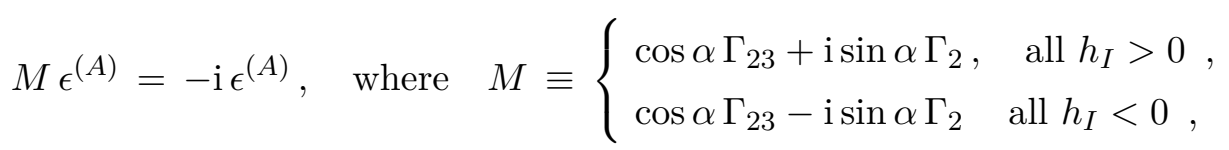

and one can verify that in both cases $M^{2}=-1$. We thus preserve half of the supersymmetries, corresponding to the superalgebra of a $d=1, \mathcal{N}=2$ SCFT. 
Notice that the squared norms of these Killing spinors are given by

$$
\zeta^{\dagger} \zeta=\tilde{\zeta}^{\dagger} \tilde{\zeta}=H^{1 / 4}
$$

which are proportional to the square root of the warp factor, as expected from the general discussion in section 2.5. We then calculate that on the spindle we have the Killing vector bilinear

$$
\frac{\mathrm{i}}{\bar{\zeta}^{\dagger} \zeta}\left(\zeta^{\dagger} \gamma^{m} \gamma_{3} \zeta\right) \partial_{m}=-\frac{\mathrm{i}}{\tilde{\zeta}^{\dagger} \tilde{\zeta}}\left(\tilde{\zeta}^{\dagger} \gamma^{m} \gamma_{3} \tilde{\zeta}\right) \partial_{m}=2 \partial_{z}
$$

where here $x^{m}=(y, z)$ are coordinates on the spindle.

Using the solutions (4.25), (4.26) one can immediately deduce the following behaviour at the poles $y_{1}, y_{2}$ in the three cases, where the statements below apply to both $\zeta$ and $\tilde{\zeta}$ :

- Case A, Twist: $\zeta^{+}\left(y_{1}\right)=0, \zeta^{+}\left(y_{2}\right)=0$, with $\zeta^{-} \neq 0$ everywhere.

- Case B, Anti-Twist: $\zeta^{-}\left(y_{1}\right)=0$ and non-vanishing everywhere else, $\zeta^{+}\left(y_{2}\right)=0$ and non-vanishing everywhere else.

- Case C, Twist: $\zeta^{-}\left(y_{1}\right)=0, \zeta^{-}\left(y_{2}\right)=0$, with $\zeta^{+} \neq 0$ everywhere.

Note that in the gauge we are using for $A^{(I)}$, the R-symmetry gauge field is not regular at either pole located at $y=y_{1}$ or $y=y_{2}$. However, we may obtain a smooth gauge field at $y=y_{1}$ via the gauge transformation $A^{R} \rightarrow A_{(0)}^{R}=A^{R}-\mathrm{d} z+\frac{\eta_{1}}{n_{1}} \mathrm{~d} \varphi$. Here we have introduced $\varphi=\frac{2 \pi}{\Delta z} z$, with $\Delta \varphi=2 \pi$. This gauge transformation will act on the spinor, which is only charged under the R-symmetry, and replace the $\mathrm{e}^{\frac{\mathrm{i} z}{2}}$ phase in (4.25) or (4.26) with $\mathrm{e}^{\frac{\mathrm{i} \eta_{1} \varphi}{2 n_{1}}}$. Noting that the frame we are using is the analogue of the hatted frame in section 2 (which is the frame that is not regular at $y=y_{1}$ ), we see that this behaviour is in agreement with (2.26). Recall here that for the twist case A, $\zeta_{+}\left(y_{1}\right)=0$ and $\eta_{1}=-1$, while for the anti-twist case B and twist case C, $\zeta_{-}\left(y_{1}\right)=0$ and $\eta_{1}=+1$ (where here and in the following the same formulas hold also for $\tilde{\zeta})$. It then follows from $(4.25),(4.26)$ that in the locally Euclidean frame and in the new gauge, for the twist case $\mathrm{A}$ we have $\zeta_{+} \sim \mathrm{e}^{-\frac{i \varphi}{n_{1}}} \rho_{1}$, where $\rho_{1}$ is the radial coordinate at $y=y_{1}$, while for the anti-twist case B and twist case $\mathrm{C}$ we instead have $\zeta_{-} \sim \mathrm{e}^{+\frac{\mathrm{i} \varphi}{n_{1}}} \rho_{1}$. In both cases we have a simple holomorphic zero of the spinor to leading order at the pole $\rho_{1}=0$. Similar comments apply, mutatis mutandis, to the behaviour of the spinors at the other pole located at $y=y_{2}$.

Finally, notice that the spinors (4.25), (4.26) are charged under azimuthal rotations of the spindle, due to the phase $\mathrm{e}^{\mathrm{i} z / 2}$. We consider the following three-parameter family of local gauge transformations on the gauge fields

$$
\tilde{A}^{(I)} \equiv A^{(I)}+\mathrm{s}^{I} \mathrm{~d} z=\rho_{I}(y) \mathrm{d} \varphi, \quad \rho_{I}(y) \equiv\left(\frac{y}{h_{I}}+\mathrm{s}^{I}\right) \frac{\Delta z}{2 \pi},
$$

where the $\mathbf{s}^{I}$ are constants satisfying the constraint

$$
\mathrm{s}^{1}+\mathrm{s}^{2}+\mathrm{s}^{3}+\mathrm{s}^{4}=-2 .
$$


The latter ensures that the R-symmetry gauge field transforms to $\tilde{A}^{R}=A^{R}-\mathrm{d} z$, which cancels the phase $\mathrm{e}^{\mathrm{i} z / 2}$ in the spinor as the spinor has charge $\frac{1}{2}$ under $A^{R}$. Using (4.16) one can check that

$$
\left.\tilde{A}^{R}\right|_{y=y_{1}}=-\frac{\eta_{1}}{n_{1}} \mathrm{~d} \varphi,\left.\quad \tilde{A}^{R}\right|_{y=y_{2}}=-\frac{\eta_{2}}{n_{2}} \mathrm{~d} \varphi
$$

This again agrees with the general conditions in section 2 we derived earlier, where in particular recall that our twist case $\mathrm{A}$ is a charge conjugate of the twist considered in section 2 .

\section{M5-branes}

In this section we will consider the case of M5-branes wrapping a spindle. The relevant solutions were constructed in a $\mathrm{U}(1)^{2}$ gauged supergravity in $D=7$ and then uplifted to $D=11$ supergravity on an $S^{4}$ in [6]. There it was shown that only the twist case is allowed. Here we explicitly construct the $D=7$ Killing spinors and confirm that they indeed have the structure that we elucidated in section 2 .

\subsection{The $D=7$ gauged supergravity model}

As in [6] the $\mathrm{U}(1)^{2}$ gauged supergravity model that we shall consider has a Lagrangian given by

$$
\mathcal{L}=\sqrt{-g}\left[R-\mathcal{V}-\frac{1}{2} \partial_{\mu} \vec{\varphi} \cdot \partial^{\mu} \vec{\varphi}-\frac{1}{4} \sum_{i=1}^{2}\left(X^{(I)}\right)^{-2}\left(F^{(I)}\right)^{2}\right]
$$

where $A^{(I)}, I=1,2$, are two $\mathrm{U}(1)$ gauge fields with field strengths $F^{(I)}=\mathrm{d} A^{(I)}$. There are two real and canonically normalized scalar fields, $\vec{\varphi}=\left(\varphi_{1}, \varphi_{2}\right)$ in terms of which

$$
X^{(1)}=\mathrm{e}^{-\frac{1}{\sqrt{2}} \varphi_{1}-\frac{1}{\sqrt{10}} \varphi_{2}} \equiv \mathrm{e}^{2 \lambda_{1}}, \quad X^{(2)}=\mathrm{e}^{+\frac{1}{\sqrt{2}} \varphi_{1}-\frac{1}{\sqrt{10}} \varphi_{2}} \equiv \mathrm{e}^{2 \lambda_{2}}
$$

where we have also introduced the scalars $\lambda_{1,2}$ for convenience. The scalar potential is given by

$$
\mathcal{V}=\frac{1}{2} \mathrm{e}^{-8 \lambda_{1}-8 \lambda_{2}}-2 \mathrm{e}^{-2 \lambda_{1}-4 \lambda_{2}}-2 \mathrm{e}^{-4 \lambda_{1}-2 \lambda_{2}}-4 \mathrm{e}^{2 \lambda_{1}+2 \lambda_{2}}
$$

Using the results of [31], any solution to the equations of motion with $F^{(I)} \wedge F^{(J)}=0$ will give rise to a solution of $D=7$ maximal gauged supergravity which can then be uplifted to $D=11$ on $S^{4}[23,32]$. In particular, the vacuum $\mathrm{AdS}_{7}$ solution, which has radius 2 , uplifts to the $\mathrm{AdS}_{7} \times S^{4}$ solution dual to the $d=6 \mathcal{N}=(0,2)$ SCFT. 
A solution will be supersymmetric provided that there are solutions to the Killing spinor equations [31]

$$
\begin{aligned}
0= & {\left[\nabla_{\mu}+\frac{1}{2}\left(A_{\mu}^{(1)} \hat{\Gamma}^{12}+A_{\mu}^{(2)} \hat{\Gamma}^{34}\right)+\frac{1}{4} \mathrm{e}^{-4 \lambda_{1}-4 \lambda_{2}} \Gamma_{\mu}+\frac{1}{2} \Gamma_{\mu} \not \partial\left(\lambda_{1}+\lambda_{2}\right)\right.} \\
& \left.+\frac{1}{4} \mathrm{e}^{-2 \lambda_{1}}\left(F_{\mu \nu}^{(1)} \Gamma^{\nu}\right) \hat{\Gamma}_{12}+\frac{1}{4} \mathrm{e}^{-2 \lambda_{2}}\left(F_{\mu \nu}^{(2)} \Gamma^{\nu}\right) \hat{\Gamma}_{34}\right] \epsilon \\
0= & {\left[\not \partial\left(3 \lambda_{1}+2 \lambda_{2}\right)-\left(\mathrm{e}^{2 \lambda_{1}}-\mathrm{e}^{-4 \lambda_{1}-4 \lambda_{2}}\right)+\frac{1}{4} \mathrm{e}^{-2 \lambda_{1}}\left(\Gamma^{\mu \nu} F_{\mu \nu}^{(1)}\right) \hat{\Gamma}_{12}\right] \epsilon, } \\
0= & {\left[\not \partial\left(2 \lambda_{1}+3 \lambda_{2}\right)-\left(\mathrm{e}^{2 \lambda_{2}}-\mathrm{e}^{-4 \lambda_{1}-4 \lambda_{2}}\right)+\frac{1}{4} \mathrm{e}^{-2 \lambda_{2}}\left(\Gamma^{\mu \nu} F_{\mu \nu}^{(2)}\right) \hat{\Gamma}_{34}\right] \epsilon . }
\end{aligned}
$$

Here $\epsilon$ is a $\operatorname{Spin}(1,6)$ spinor transforming in the 4 of the $\mathrm{SO}(5)$ R-symmetry, and is acted on by both the $D=7$ gamma matrices $\Gamma_{\mu}, \mu=0, \ldots, 6$, and the gamma matrices $\hat{\Gamma}_{A}$, $A=1, \ldots, 5$, which are associated with $\mathrm{SO}(5)$. We also note that solutions of minimal $D=7$ gauged supergravity require $A^{(1)}=A^{(2)}$ and $\lambda_{1}=\lambda_{2}$. Finally, the R-symmetry gauge field, normalized as in section 2 , is given by ${ }^{17}$

$$
A^{R} \equiv-\left(A^{(1)}+A^{(2)}\right) \text {. }
$$

\subsection{Supergravity solution and Killing spinors}

The local supersymmetric $\mathrm{AdS}_{5} \times \Sigma$ solutions discussed in [6] are given $^{18}$ by

$$
\begin{aligned}
& \mathrm{d} s_{7}^{2}=(y H)^{1 / 5}\left[\mathrm{~d} s_{\mathrm{AdS}_{5}}^{2}+\frac{y}{P} \mathrm{~d} y^{2}+\frac{P}{4 H} \mathrm{~d} z^{2}\right], \\
& A^{(I)}=-\frac{y^{2}}{h_{I}} \mathrm{~d} z, \quad X^{(I)}=\mathrm{e}^{2 \lambda_{I}}=\frac{(y H)^{2 / 5}}{h_{I}},
\end{aligned}
$$

where $\mathrm{d} s_{\mathrm{AdS}_{5}}^{2}$ is a unit radius metric, and

$$
h_{I}=y^{2}+q_{I}, \quad H=h_{1} h_{2}, \quad P=H-4 y^{3} .
$$

The solutions are parametrized by two constants $q_{I}, I=1,2$. Note that we can write the field strength as

$$
\left(X^{(I)}\right)^{-2} F^{(I)}=-\frac{4 q_{I}}{(y H)^{1 / 2}} \widetilde{\operatorname{vol}}_{\Sigma}
$$

where $\widetilde{\operatorname{vol}}(\Sigma)$ is the volume form associated to the conformally rescaled spindle metric $\widetilde{\mathrm{d} s^{2}} \Sigma=(y H)^{1 / 5} \mathrm{~d} s_{\Sigma}^{2}$.

The conditions that need to be imposed in order to obtain $\mathrm{AdS}_{5} \times \Sigma$ solutions, with $\Sigma$ a compact spindle parametrized by $(y, z)$, were described in detail in [6]. One requires $y>0, h_{I}>0, H>0$. We further require $q_{1} q_{2}<0$ and the quartic polynomial $P$ to have four real roots, with the middle two, $y_{1}, y_{2}$, both being positive. Choosing $y \in\left[y_{1}, y_{2}\right]$ then

\footnotetext{
${ }^{17}$ Note that this has the opposite sign to that defined in [6].

${ }^{18}$ Note that we have slightly changed the notation compared with [6], so that it is similar to previous sections, and we are also using a different gauge. We also note that we should identify the parameters $n_{+}, n_{-}$in [6] with $n_{1}, n_{2}$, respectively, below.
} 
gives $\mathrm{AdS}_{5} \times \Sigma$ solutions within the twist class, with no solutions in the anti-twist class. The condition $q_{1} q_{2}<0$ also shows that there are no such solutions lying within minimal $D=7$ gauged supergravity, as also pointed out in [6]. Similar to previous cases we can derive the useful result that at the roots $y=y_{1}, y_{2}$ we have

$$
\left[-\frac{3}{2}+\frac{y_{1}^{2}}{4} \sum_{I=1}^{2} \frac{1}{h_{I}\left(y_{1}\right)}\right] \frac{\Delta z}{2 \pi}=\frac{1}{n_{1}}, \quad\left[-\frac{3}{2}+\frac{y_{2}^{2}}{4} \sum_{I=1}^{2} \frac{1}{h_{I}\left(y_{2}\right)}\right] \frac{\Delta z}{2 \pi}=-\frac{1}{n_{2}},
$$

with the signs on the right hand associated with $P^{\prime}\left(y_{1}\right)>0$ and $P^{\prime}\left(y_{2}\right)<0$ and the fact that we only have the twist case. As in [6] the gauge field fluxes through the spindle are given by

$$
\begin{aligned}
Q_{I} & \equiv \frac{1}{2 \pi} \int_{\Sigma} F^{(I)}=-\left(\frac{y_{2}^{2}}{h_{I}\left(y_{2}\right)}-\frac{y_{1}^{2}}{h_{I}\left(y_{1}\right)}\right) \frac{\Delta z}{2 \pi}, \\
& \equiv \frac{p_{I}}{n_{1} n_{2}}, \quad p_{I} \in \mathbb{Z} .
\end{aligned}
$$

With the definition (5.5), the total R-symmetry flux is then calculated to by

$$
Q^{R} \equiv-\left(Q_{1}+Q_{2}\right)=-\chi(\Sigma)
$$

corresponding to the twist case only.

Here we would like to present explicit expressions for the $D=7$ Killing spinors, which were not given in [6]. We choose the following orthonormal frame

$$
e^{a}=(y H)^{1 / 10} \bar{e}^{a}, \quad e^{5}=\frac{y^{3 / 5} H^{1 / 10}}{P^{1 / 2}} \mathrm{~d} y, \quad e^{6}=\frac{y^{1 / 10} P^{1 / 2}}{2 H^{2 / 5}} \mathrm{~d} z,
$$

with $\bar{e}^{a}, a=0, \ldots, 4$ an orthonormal frame for the unit radius metric on $\operatorname{AdS}_{5}$. We use explicit $D=7$ gamma matrices given by

$$
\Gamma_{a}=\beta_{a} \otimes \gamma_{3}, \quad \Gamma_{5}=1 \otimes \gamma_{1}, \quad \Gamma_{6}=1 \otimes \gamma_{2},
$$

where $\beta_{a}$ are five-dimensional gamma matrices given by $\beta_{0}=\mathrm{i} \sigma^{2} \otimes 1, \beta_{1}=\sigma^{1} \otimes 1$, $\beta_{2}=\sigma^{3} \otimes \sigma^{1}, \beta_{3}=\sigma^{3} \otimes \sigma^{2}, \beta_{4}=\sigma^{3} \otimes \sigma^{3}$, and $\gamma_{1}=\sigma^{1}, \gamma_{2}=\sigma^{2}$ are again gamma matrices for the spindle directions with $\gamma_{3}=\sigma^{3}$. We can also choose an explicit set of $\mathrm{SO}(5)$ gamma matrices as follows: $\hat{\Gamma}_{1}=-\mathrm{i} \beta_{0}, \hat{\Gamma}_{a}=\beta_{a-1}$, for $a=2, \ldots, 5$.

We next introduce the Killing spinor equation for $\mathrm{AdS}_{5}$, which reads

$$
\nabla_{\mu} \vartheta=\frac{\mathrm{k}}{2} \beta_{\mu} \vartheta
$$

with $\mathrm{k}= \pm 1$. For each value of $\mathrm{k}$ the solution admits four independent solutions, which we label $\vartheta_{\mathrm{k}}^{(A)}, A=1, \ldots, 4$ (see appendix B). To describe the $D=7$ Killing spinors, we need two more ingredients. The first is the common eigenvectors of the $\mathrm{SO}(5)$ matrices $\hat{\Gamma}_{12}$ and $\hat{\Gamma}_{34}$ that have the same eigenvalue. There are two of them, which we label $u_{ \pm}$, satisfying

$$
\Gamma_{12} u_{ \pm}=\Gamma_{34} u_{ \pm}= \pm \mathrm{i} u_{ \pm}
$$


The second ingredient is the charge conjugation matrix on the spindle $\Sigma$, which we take to be $B=\gamma_{1}$, so that $B\left(\gamma_{1,2}\right)^{*} B^{-1}=\gamma_{1,2}$, where $\gamma_{1,2}$ are two-dimensional gamma matrices on $\Sigma$, given above. We then define the charge conjugate of a two-dimensional spinor $\zeta$ on the spindle as $\zeta^{c}=B \zeta^{*}$.

The $\mathrm{AdS}_{5} \times \Sigma$ solutions preserve $1 / 2$ of the supersymmetry and we can now write the eight Killing spinors as

$$
\epsilon_{-}^{(A)}=\vartheta_{-}^{(A)} \otimes \zeta \otimes u_{-}, \quad \text { and } \quad \epsilon_{+}^{(A)}=\vartheta_{+}^{(A)} \otimes \zeta^{c} \otimes u_{+},
$$

where $\zeta$ is the two-dimensional spinor on the spindle given by

$$
\begin{aligned}
\zeta \equiv\left(\begin{array}{c}
\zeta_{+} \\
\zeta_{-}
\end{array}\right) & =\mathrm{e}^{-\frac{3 \mathrm{i} z}{4}} \frac{y^{1 / 20}}{\sqrt{2} H^{1 / 5}}\left(\begin{array}{c}
{\left[\sqrt{H}+2 y^{3 / 2}\right]^{1 / 2}} \\
-\left[\sqrt{H}-2 y^{3 / 2}\right]^{1 / 2}
\end{array}\right) \\
& =\mathrm{e}^{-\frac{3 \mathrm{i} z}{4}}(y H)^{1 / 20}\left(\begin{array}{c}
\cos \frac{\alpha}{2} \\
-\sin \frac{\alpha}{2}
\end{array}\right)
\end{aligned}
$$

where the function $\alpha=\alpha(y)$ satisfies

$$
\sin \alpha=\frac{P^{1 / 2}}{H^{1 / 2}}, \quad \cos \alpha=\frac{2 y^{3 / 2}}{H^{1 / 2}} .
$$

In particular then the spinors $\epsilon^{(A)}$ given in (5.16) satisfy

$$
M_{-} \epsilon_{-}^{(A)}=-\mathrm{i} \epsilon_{-}^{(A)}, \quad M_{+} \epsilon_{+}^{(A)}=+\mathrm{i} \epsilon_{+}^{(A)},
$$

where

$$
M_{ \pm}=\cos \alpha \Gamma_{56} \mp \mathrm{i} \sin \alpha \Gamma_{5},
$$

and one can verify that $M^{2}=-1$. We thus preserve half of the supersymmetries, corresponding to a $4 d, \mathcal{N}=1 \mathrm{SCFT}$. The squared norm of $\zeta$ is

$$
\zeta^{\dagger} \zeta=(y H)^{1 / 10}
$$

which is proportional to the square root of the warp factor, as expected from the general discussion in section 2.5. We then calculate that on the spindle we have the Killing vector bilinear

$$
\frac{\mathrm{i}}{\zeta^{\dagger} \zeta}\left(\zeta^{\dagger} \gamma^{m} \gamma_{3} \zeta\right) \partial_{m}=2 \partial_{z}
$$

where here $x^{m}=(y, z)$ are coordinates on the spindle.

As noted above, the supergravity solutions constructed in [6] all lie within the twist class. In particular $y \in\left[y_{1}, y_{2}\right]$ with $y_{1}, y_{2}>0$ and satisfying $H\left(y_{i}\right)=4 y_{i}^{3}$. Correspondingly we see from (5.17) that at both poles of the spindle one has $\zeta_{-}\left(y_{1,2}\right)=0$, with $\zeta_{+}\left(y_{1,2}\right) \neq 0$, in precise alignment with section 2 .

Note that in the gauge we are using for $A^{(I)}$, the R-symmetry gauge field is not regular at either pole located at $y=y_{1}$ or $y=y_{2}$. Using (5.9) we can obtain a smooth gauge field at $y=y_{1}$ via the gauge transformation: $-A^{R} \rightarrow-A_{(0)}^{R}=-A^{R}+\frac{3}{2} \mathrm{~d} z+\frac{1}{n_{1}} \mathrm{~d} \varphi$. Here 
we have introduced $\varphi=\frac{2 \pi}{\Delta z} z$, with $\Delta \varphi=2 \pi$. This gauge transformation will act on the spinor, which is only charged under the R-symmetry, and replace the $\mathrm{e}^{-\frac{3 \mathrm{i} z}{4}}$ phase in (5.17) with $\mathrm{e}^{\frac{\mathrm{i} \varphi}{2 n_{1}}}$. Noting that the frame we are using is the analogue of the hatted frame in section 2 (which is the frame that is not regular at $y=y_{1}$ ), we see that this behaviour is in agreement with (2.26). Furthermore, we observe from (5.17) that in this frame and the new gauge, we have $\zeta_{-} \sim \mathrm{e}^{\frac{i \varphi}{2 n_{1}}} \rho_{1}$, where $\rho_{1}$ is the radial coordinate at $y=y_{1}$. Converting the spinor to the locally Euclidean frame we then have $\zeta_{-} \sim \mathrm{e}^{\frac{i \varphi}{n_{1}}} \rho_{1}$, and we see that it has a simple holomorphic zero at the pole $\rho_{1}=0$. Similar comments apply, mutatis mutandis, to the behaviour of the spinors at the other pole located at $y=y_{2}$.

We can also define the following one-parameter family of gauge transformations

$$
\tilde{A}^{(I)} \equiv A^{(I)}+\mathbf{s}^{I} \mathrm{~d} z=\rho_{I}(y) \mathrm{d} \varphi,
$$

where the $\mathbf{s}^{I}$ are constants satisfying the constraint

$$
\mathrm{s}^{1}+\mathrm{s}^{2}=+\frac{3}{2}
$$

Here we have introduced the functions

$$
\rho_{I}(y) \equiv\left(-\frac{y^{2}}{h_{I}}+\mathbf{s}^{I}\right) \frac{\Delta z}{2 \pi} .
$$

These functions, which play a role in the field theory analysis, generalize the analogous quantities in [6] (which had $\mathbf{s}^{1}=\mathbf{s}^{2}=3 / 4$ ), as we remark upon in the next subsection. In the frame that we are using and in the tilded gauge the spinors are independent of the $\varphi$ coordinate. From the results of [6] one can also check that

$$
\left.\tilde{A}^{R}\right|_{y=y_{1}}=\frac{1}{n_{1}} \mathrm{~d} \varphi,\left.\quad \tilde{A}^{R}\right|_{y=y_{2}}=-\frac{1}{n_{2}} \mathrm{~d} \varphi .
$$

This again agrees with the general conditions (2.49) we derived for the twist case earlier.

\subsection{Comparison with field theory}

The $\mathrm{AdS}_{5} \times \Sigma$ solutions were uplifted on an $S^{4}$ from $D=7$ to $D=11$ supergravity in [6]. While the $D=11$ solutions still have orbifold singularities, it is straightforward to carry out flux quantization and calculate the $a$ central charge for the dual $d=4$ SCFT, and the result [6] is given by

$$
a=\frac{3 p_{1}^{2} p_{2}^{2}\left(\mathbf{s}+p_{1}+p_{2}\right)}{8 n_{2} n_{1}\left(n_{2}-p_{1}\right)\left(p_{2}-n_{2}\right)\left[\mathbf{s}+2\left(p_{1}+p_{2}\right)\right]^{2}} N^{3},
$$

where

$$
\mathbf{s}=\sqrt{\left(p_{1}+p_{2}\right)^{2}-12\left(n_{2}-p_{1}\right)\left(n_{2}-p_{2}\right)} .
$$

The supergravity solution is valid for $n_{2}>n_{1}$ as well as $p_{1}<0$ or $p_{1}>n_{1}+n_{2}$ (and hence from (5.11) we have $p_{2}>0$ or $p_{2}<0$, respectively), which ensures that $a>0$. 
The field theory calculations in [6] were carried out using the tilded gauge fields in (5.23) with $\mathbf{s}^{1}=\mathbf{s}^{2}=3 / 4$, but is straightforward to generalize that calculation to an arbitrary gauge with $\mathbf{s}^{1}+\mathbf{s}^{2}=+\frac{3}{2}$ (which recall is required to ensure that the spinors are uncharged with respect to $\partial_{\varphi}$ ), much as in section 3 for the case of D3-branes. Doing so has no effect on the central charge and mixing parameter $\epsilon$, both of which are gauge-invariant quantities, but it does give rise to more general values for the extremized trial R-charge parameters $\Delta_{1}^{*}, \Delta_{2}^{*}$. It would be of interest to understand why the gauge $\mathbf{s}^{1}=\mathbf{s}^{2}=3 / 4$ leads to the simple result of [6] that $\Delta_{1}^{*}=\Delta_{2}^{*}=1$.

The field theory calculation for the anti-twist can also be carried out in a straightforward manner. One finds that in this case it is certainly possible to find fluxes on the spindle that give rise to a positive central charge. However, these cases seem to be obstructed in the sense that there are no dual solutions within our gauged supergravity construction.

\section{Final comments}

In this paper we have given a general proof that supersymmetric geometries involving a spindle $\Sigma$ with azimuthal symmetry fall into two classes: the twist and anti-twist, where the quantized magnetic flux through the spindle is necessarily given by (1.1). Previous constructions of $\mathrm{AdS}_{3} \times \Sigma \mathrm{D} 3$-brane and $\mathrm{AdS}_{2} \times \Sigma \mathrm{M} 2$-brane solutions are all in the antitwist class, but in this paper we have shown in both cases that there are also twist solutions. The $\mathrm{AdS}_{5} \times \Sigma \mathrm{M} 5$-brane solutions in [6] are also twist solutions, and interestingly in contrast to the D3-brane and M2-brane cases here we do not find anti-twist solutions at all, at least in the gauged supergravity construction presented. Notice that a common feature in all cases is that we do not find twist solutions in minimal gauged supergravity, where there is effectively only a single magnetic charge; although for D3-branes and M2-branes there are anti-twist solutions in minimal gauged supergravity $[1,3]$, that uplift to $D=10$ and $D=11$ supergravity on general regular Sasaki-Einstein manifolds, respectively.

In all cases the twist solutions have a natural physical interpretation as the IR limit of branes wrapped on $\Sigma$ holomorphically embedded in a Calabi-Yau $(n+1)$-fold, where $n=2,3$ or 4 , in the cases of M5-branes, D3-branes or M2-branes, respectively. The CalabiYau $(n+1)$-fold is the total space of a $\mathbb{C}^{n}$ bundle over $\Sigma$, given by

$$
\bigoplus_{i=1}^{n} \mathcal{O}\left(p_{i}\right) \rightarrow \Sigma
$$

where $p_{i} \in \mathbb{Z}$ give the quantized magnetic charges of the $n \mathrm{U}(1)$ gauge fields in the gauged supergravity constructions. The first Chern class of the space (6.1) is zero ${ }^{19}$ if and only if

$$
\sum_{i=1}^{n} \frac{p_{i}}{n_{1} n_{2}}+\chi=0
$$

\footnotetext{
${ }^{19}$ Note that a little care is required when identifying the $p_{i}$ in this discussion with the $p_{i}$ in the twist solutions in the bulk of the paper. In section 3, for the D3-brane twist solutions they are the same. In section 4, for the case of the M2-branes they are the same for the twist case A, but for the twist case C the Calabi-Yau is instead $\bigoplus_{i=1}^{n} \mathcal{O}\left(-p_{i}\right) \rightarrow \Sigma$ with $\sum_{i=1}^{n} \frac{-p_{i}}{n_{1} n_{2}}+\chi=0$, as it is also for the M5-brane solutions in section 5 .
} 
where $\chi=\chi(\Sigma)$ is the Euler number of the spindle, which is precisely the twist R-symmetry flux condition in (1.1). One starts with a Minkowski $\times \mathrm{CY}_{n}$ solution to string theory or M-theory, appropriately, and wraps $N$ D3-branes, M2-branes or M5-branes over the holomorphically embedded $\Sigma \subset \mathrm{CY}_{n}$. The resulting low-energy theory on the Minkowski space part of the worldvolume is supersymmetric, utilizing a topological twist. The AdS twist solutions we have constructed in all cases are then naturally interpreted as the near-horizon limits of these wrapped brane configurations, which correspondingly flow to superconformal field theories in the IR. For the D3-brane and M5-brane solutions we have presented dual field theory calculations that corroborate this picture. It is also interesting to highlight that for the D3-brane and M5-brane spindle twist solutions, we showed that the expression for the central charge exactly reproduces the central charge for known supergravity solutions describing D3-brane and M5-branes wrapping an $S^{2}$ with a topological twist, upon setting $n_{1}=n_{2}=1$. This is despite the fact that we cannot take a smooth limit of the spindle solutions to get the topologically twisted solutions.

However, this discussion also raises a number of interesting open questions. Firstly, note that (6.1) is a Calabi-Yau for any values of $p_{i} \in \mathbb{Z}$ that satisfy the constraint (6.2). Since $\chi>0$, certainly the $p_{i}$ cannot all be positive. However, we only find AdS solutions when the $p_{i}$ satisfy additional inequalities. For example, the D3-brane solutions exist only when $n_{2}>n_{1}$ and two of the three $p_{i}(i=1,2,3)$ are negative, while one is positive. Similarly, in the M5-brane case also $n_{2}>n_{1}$ and one of $p_{1}$ or $p_{2}$ must be negative, with the other positive. What happens to the field theories on the wrapped branes in the IR when the charges do not satisfy these conditions? We have no candidate AdS solutions, and it's possible the theories do not flow to a superconformal fixed point. It would be interesting to understand these conditions on the magnetic charges from a purely field theory point of view. Notice that whenever $p_{i}>0$ in (6.1), for a given $i$, the corresponding normal bundle direction has non-trivial holomorphic sections and hence the wrapped brane has corresponding linear deformations. The existence (or not) of this moduli space presumably plays some role in the IR behaviour.

More puzzling is the corresponding interpretation of the anti-twist solutions. One can formally still write down a complex manifold (6.1) in this case, but it is not Calabi-Yau, as the first Chern class is not zero. However, deleting the zero section gives a complex cone that does have zero first Chern class; indeed this is a necessary condition for all GK geometries [33], which are relevant for the D3-brane $\mathrm{AdS}_{3} \times Y_{7}$ and $\mathrm{M} 2$-brane $\mathrm{AdS}_{2} \times Y_{9}$ solutions. Thus there is a Calabi-Yau cone geometry, but there is no "blown-up" Calabi-Yau geometry with $\Sigma$ a holomorphic curve for the anti-twist. The AdS anti-twist solutions strongly suggest an interpretation in terms of $N$ D3-branes or $N$ M2-branes wrapping $\Sigma$, and the field theory calculation in section 3.4 assumes this in the D3-brane case and then correctly reproduces the supergravity central charge. But there is not a UV interpretation as $N$ branes wrapping a holomorphic curve in a Calabi-Yau, as there is for the twist solutions. On the other hand, the anti-twist solutions have Killing spinors which are sections of nontrivial bundles, and clearly preserve supersymmetry differently than for standard wrapped branes. It remains an interesting open problem to identify a UV geometry for this case. 
In this paper, we have also explicitly calculated the Killing spinors for various $\operatorname{AdS} \times \Sigma$ solutions of gauged supergravity and, by constructing vector spinor bilinears, elucidated the associated supersymmetry algebra. At the level of the gauged supergravity, the rotation symmetry of the spindle enters the supersymmetry algebra as an R-symmetry and this is in contrast to the case of the topological twist where it does not. After uplifting to $D=10,11$ supergravity the internal space of the AdS solutions has an R-symmetry Killing vector which appears in the SCFT algebra. In the case of the topological twist, this is just an R-symmetry Killing vector of the space one uplifts upon, and dual to an R-symmetry of the brane world-volume theory. For the spindle solutions, it is instead a linear combination of an R-symmetry Killing vector of the space one uplifts upon with the Killing vector of the spindle, and from a dual point of view corresponds to the fact that in this case there is a mixing of the R-symmetry in the brane world-volume theory.

Supergravity solutions associated with D4-branes wrapping a spindle were recently constructed in [2]. Supersymmetric $\mathrm{AdS}_{4} \times \Sigma$ solutions were constructed in a $D=6$ gauged supergravity theory and then uplifted on a hemisphere to obtain solutions of massive type IIA supergravity. All of the solutions presented lie within the twist class, consistent with the general result of this paper, and it appears that there are no solutions within the antitwist case. Once again it would be interesting to know why that is. It was also shown that the rotation symmetry of the spindle enters the supersymmetry algebra within the gauged supergravity theory, in the same way that we have shown in this paper for the case of D3-, M2- and M5-brane solutions.

Our general result concerning the existence of just two classes of solutions for the spindle, the twist and the anti-twist, crucially relied on the assumption that the spindle has an azimuthal symmetry. It would be of much interest to construct analogous supergravity solutions for spindles without such symmetry, or more generally, for orbifold Riemann surfaces of arbitrary genus and with an arbitrary number of orbifold points. In the case of D3-branes and M2-branes one can aim to make progress using the recent advances in understanding GK geometry [34-39].

\section{Acknowledgments}

We would like to thank Nikolay Bobev, Dario Martelli and Nikita Nekrasov for helpful discussions. This work was supported in part by STFC grants ST/T000791/1 and $\mathrm{ST} / \mathrm{T} 000864 / 1$. JPG is supported as a Visiting Fellow at the Perimeter Institute.

\section{A Spheres from spindles}

\section{A.1 $\quad \operatorname{AdS}_{3} \times S^{2}$ solutions as a limit}

Here we show how the $\mathrm{AdS}_{3} \times S^{2}$ solutions of [21], associated with the usual topological twist, can be obtained as a limit of the local class of solutions given in (3.6).

The solutions of [21] have both the warp factor $H$ and the scalar fields constant, which clearly cannot be obtained just by tuning the parameters $q_{I}$. We thus need to take a scaling 
limit. We begin by writing

$$
y=\alpha+\lambda x, \quad q_{I}=-\alpha+r_{I}+s \lambda^{2},
$$

where $\alpha, r_{I}, s$ and $\lambda$ are constants. Taking $\lambda \rightarrow 0$ one finds that

$$
H \longrightarrow r_{1} r_{2} r_{3}
$$

which is constant as desired. Moreover, since in the scaling limit $\mathrm{d} y^{2} \rightarrow \lambda^{2} \mathrm{~d} x^{2}$ we want $P$ to scale with $\lambda^{2}$ in the limit, and this will ultimately lead to a round $S^{2}$ in the compact directions. This is obtained by setting

$$
\alpha=\sqrt{r_{1} r_{2} r_{3}}, \quad s=\frac{1-\left(r_{1}+r_{2}+r_{3}\right)}{2 \sqrt{r_{1} r_{2} r_{3}}},
$$

and moreover the parameters $r_{I}$ must be constrained to satisfy

$$
\frac{\alpha}{2} \sum_{I=1}^{3} r_{I}^{-1}=1 \quad \Leftrightarrow \quad r_{1} r_{2}+r_{1} r_{3}+r_{2} r_{3}=2 \sqrt{r_{1} r_{2} r_{3}} .
$$

After redefining the azimuthal coordinate via

$$
z=\frac{1}{4 \lambda s} \phi
$$

we obtain the metric

$$
\mathrm{d} s^{2}=F^{1 / 3}\left(\mathrm{~d} s_{\mathrm{AdS}_{3}}^{2}+G \mathrm{~d} s_{S^{2}}^{2}\right),
$$

where $\mathrm{d} s_{S^{2}}^{2}$ is a round metric on $S^{2}$ in spherical coordinates, after setting $x=\cos \theta$. The functions of the parameters $F$ and $G$ are given by

$$
F=\alpha^{2}=r_{1} r_{2} r_{3}, \quad G=(8 \alpha s)^{-1} .
$$

Using the same coordinate transformations, as well as a gauge transformation to remove terms in the gauge fields that are singular as $\lambda \rightarrow 0$, we obtain the gauge fields and (constant) scalars given by

$$
\begin{aligned}
A^{(I)} & =\frac{2 F^{1 / 2} G}{r_{I}}\left(1-\frac{F^{1 / 2}}{r_{I}}\right) \cos \theta \mathrm{d} \phi \equiv a_{I} \cos \theta \mathrm{d} \phi, \\
X^{(I)} & =\frac{F^{1 / 3}}{r_{I}} .
\end{aligned}
$$

Here we have introduced the parameters $a_{I}$, which are constrained by (A.4). Choosing the correct sign on solving that equation, one finds

$$
a_{1}+a_{2}+a_{3}=-1
$$

or equivalently

$$
Q^{R}=\frac{1}{2 \pi} \int_{S^{2}} \mathrm{~d}\left(A^{(1)}+A^{(2)}+A^{(3)}\right)=2,
$$


corresponding to a twist case. It is straightforward to check that this reproduces the $\mathrm{AdS}_{3} \times S^{2}$ solution of [21]. Moreover, from the expression for the Killing spinors given in (3.36), we see that this scaling limit will give rise to constant, anti-chiral spinors, as expected for the topological twist.

Finally, the form (A.6) of the metric leads to a simple expression for the central charge of the dual two-dimensional SCFT arising from placing $d=4 N=4$ SYM theory on $S^{2}$ with a topological twist. Recall that the vacuum solution of our $D=5$ theory (3.1) is an $\mathrm{AdS}_{5}$ with unit radius, the five-dimensional Newton constant is given by $\left(G_{(5)}\right)^{-1}=$ $2 N^{2} / \pi$. Using (A.6) we can then obtain the three-dimensional Newton constant for a theory admitting a unit radius $\mathrm{AdS}_{3}$ solution: $\left(G_{(3)}\right)^{-1}=8 F^{1 / 2} G N^{2}$ and hence we deduce the $d=2$ central charge

$$
c=\frac{3}{2 G_{(3)}}=12 F^{1 / 2} G N^{2}=\frac{3 \sqrt{r_{1} r_{2} r_{3}}}{1-\left(r_{1}+r_{2}+r_{3}\right)} N^{2} .
$$

Using the relation between the parameters $a_{I}$ and the $r_{I}$ given implicitly in (A.8), as well as the constraint (A.4), it is straightforward to check that this expression agrees with the central charge of the solutions found in [21] in the case where the internal manifold is a sphere, which is also recalled in eq. (3.60).

\section{A.2 $\quad \operatorname{AdS}_{2} \times S^{2}$ solutions as a limit}

Here we show how the $\mathrm{AdS}_{2} \times S^{2}$ solutions of [40], associated with the usual topological twist and arising as the near-horizon limit of an asymptotically $\mathrm{AdS}_{4}$ black hole, can be obtained as a limit of the local class of solutions given in (4.6).

As in section A.1, both the warp factor $H$ and the scalar fields should be constant, so we need to take a scaling limit. We write

$$
y=\alpha+\lambda x, \quad q_{I}=-\alpha+r_{I}+s \lambda^{2},
$$

where $\alpha, r_{I}, s$ and $\lambda$ are constants. Taking $\lambda \rightarrow 0$ one finds that square root removed

$$
H \longrightarrow r_{1} r_{2} r_{3} r_{4},
$$

which is constant as desired. Moreover, since in the scaling limit $\mathrm{d} y^{2} \rightarrow \lambda^{2} \mathrm{~d} x^{2}$ we want $P$ to scale with $\lambda^{2}$ in the limit, and this will ultimately lead to a round $S^{2}$ in the compact directions. This is obtained by setting

$$
\alpha=\frac{\sqrt{r_{1} r_{2} r_{3} r_{4}}}{2}, \quad s=\frac{4-\left(r_{1} r_{2}+r_{1} r_{3}+r_{1} r_{4}+r_{2} r_{3}+r_{2} r_{4}+r_{3} r_{4}\right)}{4 \sqrt{r_{1} r_{2} r_{3} r_{4}}},
$$

and moreover the parameters $r_{I}$ must be constrained to satisfy

$$
\frac{\alpha}{2} \sum_{I=1}^{4} r_{I}^{-1}=1 \quad \Leftrightarrow \quad r_{1} r_{2} r_{3}+r_{1} r_{2} r_{4}+r_{1} r_{3} r_{4}+r_{2} r_{3} r_{4}=4 \sqrt{r_{1} r_{2} r_{3} r_{4}} .
$$

After redefining the azimuthal coordinate via

$$
z=\frac{1}{2 \lambda s} \phi,
$$


we obtain the metric

$$
\mathrm{d} s^{2}=F^{1 / 2}\left(\frac{1}{4} \mathrm{~d} s_{\mathrm{AdS}_{2}}^{2}+G \mathrm{~d} s_{S^{2}}^{2}\right),
$$

where $\mathrm{d} s_{S^{2}}^{2}$ is a round metric on $S^{2}$ in spherical coordinates, after setting $x=\cos \theta$.

$$
F=(2 \alpha)^{2}=r_{1} r_{2} r_{3} r_{4}, \quad G=(8 \alpha s)^{-1}
$$

Using the same coordinate transformations, as well as a gauge transformation to remove terms in the gauge fields that are singular as $\lambda \rightarrow 0$, we obtain the gauge fields and (constant) scalars given by

$$
\begin{aligned}
A^{(I)} & =\frac{F^{1 / 2} G}{r_{I}}\left(2-\frac{F^{1 / 2}}{r_{I}}\right) \cos \theta \mathrm{d} \phi, \\
X^{(I)} & =\frac{F^{1 / 4}}{r_{I}} .
\end{aligned}
$$

One can check that, the constraint (A.15) constrains the total magnetic flux to be

$$
Q^{R}=\frac{1}{2 \pi} \int_{S^{2}} \frac{1}{2} \mathrm{~d}\left(A^{(1)}+A^{(2)}+A^{(3)}+A^{(4)}\right)=2,
$$

which corresponds to a twist case. Moreover, after the scaling limit and the gauge transformation, the Killing spinors (4.25) reduce to constant, chiral spinors on the sphere, which corresponds to a standard topological twist. Although we have not matched the parameters explicitly, this should reproduce the near-horizon limit of the supersymmetric black holes given in [40]. In terms of the parameters $r_{I}$ used here, from (A.17) we deduce that the area of the horizon is $A_{\text {horizon }}=4 \pi F^{1 / 2} G$ and hence the black hole entropy reads

$$
S_{B H}=\frac{\pi}{G_{(4)}} \frac{\sqrt{r_{1} r_{2} r_{3} r_{4}}}{4-\left(r_{1} r_{2}+r_{1} r_{3}+r_{1} r_{4}+r_{2} r_{3}+r_{2} r_{4}+r_{3} r_{4}\right)},
$$

where we remind the reader that the parameters $r_{I}$ are subject to the constraint (A.15). This can be expressed in terms of magnetic charges via (A.19). Also, recalling that the vacuum solution of our $D=4$ theory (4.1) is an $\mathrm{AdS}_{4}$ with unit radius, which uplifts to $\mathrm{AdS}_{4} \times S^{7}$ dual to ABJM theory, the four-dimensional Newton constant is given by $\left(G_{(4)}\right)^{-1}=2^{3 / 2} N^{3 / 2} /(3 \pi)$.

\section{A.3 $\quad \operatorname{AdS}_{5} \times S^{2}$ solutions as a limit}

Here we show how the $\operatorname{AdS}_{5} \times S^{2}$ solutions of [41], associated with the usual topological twist, can be obtained as a limit of the local class of solutions given in (5.6).

As in appendix A.1, the solutions of [41] have both the warp factor $H$ and the scalar fields constant, so we take a scaling limit. We set

$$
y=\alpha+\lambda x, \quad q_{I}=-\alpha^{2}+r_{I}+s \lambda^{2},
$$

where $\alpha, r_{I}, s$ and $\lambda$ are constants. Taking taking $\lambda \rightarrow 0$ one finds that

$$
H \longrightarrow r_{1} r_{2}
$$


which is constant as desired. Moreover, since in the scaling limit $\mathrm{d} y^{2} \rightarrow \lambda^{2} \mathrm{~d} x^{2}$ we want $P$ to scale with $\lambda^{2}$ in the limit, and this will ultimately lead to a round $S^{2}$ in the compact directions. This is obtained by setting

$$
\alpha=\left(\frac{r_{1} r_{2}}{4}\right)^{1 / 3}, \quad s=1-\frac{r_{1}+r_{2}}{9},
$$

and moreover the parameters $r_{I}$ must be constrained to satisfy

$$
\frac{2 \alpha^{2}}{3} \sum_{I=1}^{2} r_{I}^{-1}=1 \quad \Leftrightarrow \quad r_{1}+r_{2}=3\left(2 r_{1} r_{2}\right)^{1 / 3} .
$$

After setting

$$
z=-\frac{2 \alpha}{3 \lambda s} \phi
$$

we obtain the metric

$$
\mathrm{d} s^{2}=F^{1 / 5}\left(\mathrm{~d} s_{\mathrm{AdS}_{5}}^{2}+G \mathrm{~d} s_{S^{2}}^{2}\right)
$$

where $\mathrm{d} s_{S^{2}}^{2}$ is a round metric on $S^{2}$ in spherical coordinates, after setting $x=\cos \theta$. The functions of the parameters $F$ and $G$ are given by

$$
F=4 \alpha^{4}, \quad G=(6 s)^{-1} .
$$

Using the same coordinate transformations, as well as a gauge transformation to remove terms in the gauge fields that are singular as $\lambda \rightarrow 0$, we obtain the gauge fields and (constant) scalars given by

$$
\begin{aligned}
A^{(I)} & =\frac{2 F^{1 / 2} G}{r_{I}}\left(2-\frac{F^{1 / 2}}{r_{I}}\right) \cos \theta \mathrm{d} \phi, \\
X^{(I)} & =\frac{F^{2 / 5}}{r_{I}} .
\end{aligned}
$$

One can check that, the constraint (A.25) constrains the total magnetic flux to be

$$
Q^{R}=-\frac{1}{2 \pi} \int_{S^{2}} \mathrm{~d}\left(A^{(1)}+A^{(2)}\right)=-2,
$$

which corresponds to a twist case. Moreover, after the scaling limit and the gauge transformation, the Killing spinors (5.17) reduce to constant, chiral spinors on the sphere, which corresponds to a standard topological twist. The local solution matches exactly that of [41] after setting their parameter $z$ to $\mathrm{be}^{20}$

$$
z=-1-\frac{2 G}{3 F^{1 / 2}}\left(2 r_{1}-r_{2}\right) r_{2} .
$$

Finally, the form (A.27) of the metric leads to a simple expression for the central charge of the dual four-dimensional SCFT arising from placing the $d=6, \mathcal{N}=(0,2)$

\footnotetext{
${ }^{20}$ Note that in [41] the authors set the gauge coupling $m=2$, while our conventions correspond to $m=1$. Thus, with this choice of $z$ we reproduce their solution modulo adjusting the value of the gauge coupling.
} 
theory on $S^{2}$ with a topological twist. Recalling that the vacuum solution of our $D=7$ theory (5.1) is an $\mathrm{AdS}_{7}$ with radius 2, the seven-dimensional Newton constant, is given by $\left(G_{(7)}\right)^{-1}=N^{3} /\left(6 \pi^{2}\right)$. Using (A.27) we can then obtain the five-dimensional Newton constant for a theory admitting a unit radius $\mathrm{AdS}_{5}$ solution: $\left(G_{(5)}\right)^{-1}=2 F^{1 / 2} G N^{3} /(3 \pi)$ and hence the $d=4$ central charge

$$
a=\frac{\pi}{8 G_{(5)}}=\frac{F^{1 / 2} G}{12} N^{3}=\frac{2\left(r_{1}+r_{2}\right)^{2}}{9\left[9-\left(r_{1}+r_{2}\right)\right]} N^{3} .
$$

Using the relation (A.31), as well as the constraint (A.25), it is straightforward to check that this expression agrees with the central charge of the solutions found in eq. (2.22) of [41] in the case where the internal manifold is a sphere, which we recall here for the reader's convenience:

$$
a=\frac{1-9 z^{2}+\left(1+3 z^{2}\right)^{3 / 2}}{48 z^{2}} N^{3}
$$

This central charge also arises in the limit $n_{1,2} \rightarrow 1$ of the central charge found in [6] (see the discussion at the end of section 4) for the $\mathrm{AdS}_{5}$ spindle solutions discussed in section 5 .

\section{B Killing spinors in AdS}

Here we include a few comments on AdS Killing spinors that we use in the main text (see also $[42,43])$. Using Poincaré coordinates $\bar{x}^{a}=\left(t, x_{1}, \ldots x_{d-1}, r\right)$, we can write the metric on a unit radius $\mathrm{AdS}_{d+1}$ in the form

$$
\mathrm{d} s_{\mathrm{AdS}_{d+1}}^{2}=\frac{1}{r^{2}}\left(-\mathrm{d} t^{2}+\sum_{i=1}^{d-1} \mathrm{~d} x_{i}^{2}+\mathrm{d} r^{2}\right),
$$

with an associated orthonormal frame given by

$$
\bar{e}^{0}=\frac{\mathrm{d} t}{r}, \quad \bar{e}^{1}=\frac{\mathrm{d} x_{1}}{r}, \quad \ldots, \quad \bar{e}^{d-1}=\frac{\mathrm{d} x_{d-1}}{r}, \quad \bar{e}^{d}=\frac{\mathrm{d} r}{r} .
$$

We introduce arbitrary gamma matrices $\beta_{a}$ satisfying $\left\{\beta_{a}, \beta_{b}\right\}=2 \eta_{a b}$ with signature $(-,+, \ldots,+)$. We want to construct solutions to the Killing spinor equation

$$
\nabla_{a} \vartheta=\frac{\mathrm{k}}{2} \beta_{a} \vartheta
$$

where $\mathrm{k}= \pm 1$. For each choice of $\mathrm{k}$ we can find $\mathrm{d}=2^{\lfloor(d+1) / 2\rfloor}$ solutions; half of them correspond to Poincaré supercharges $\mathcal{Q}_{\mathrm{k}}^{(\alpha)}$, and their bilinears give rise to translations in the conformal algebra, while the remaining half corresponds to conformal supercharges $\mathcal{S}_{\mathrm{k}}^{(\alpha)}$, and their bilinears give rise to special conformal transformations. We can express them in terms of the eigenvectors of $\beta_{d}$, associated with the $\mathrm{d} r / r$ component of the frame. Since $\left(\beta_{d}\right)^{2}=1$, the eigenvectors of $\beta_{d}$ come in two sets $v_{ \pm}^{(\alpha)}$, satisfying

$$
\beta_{d} v_{ \pm}^{(\alpha)}= \pm v_{ \pm}^{(\alpha)}, \quad \alpha=1, \ldots, \frac{\mathrm{d}}{2}
$$


The solutions to the Killing spinor equation (B.3) can then be written as

$$
\mathcal{Q}_{ \pm}^{(\alpha)}=\frac{1}{\sqrt{r}} v_{\mp}^{(\alpha)}, \quad \mathcal{S}_{ \pm}^{(\alpha)}=\frac{1}{\sqrt{r}} \beta_{a} \bar{x}^{a} v_{ \pm}^{(\alpha)} .
$$

We shall refer to the d solutions of (B.3), for a given $\mathrm{k}$, as $\vartheta_{\mathrm{k}}^{(A)}$, with

$$
\vartheta_{\mathrm{k}}^{(A)}=\left(\mathcal{Q}_{\mathrm{k}}^{(\alpha)}, \mathcal{S}_{\mathrm{k}}^{(\alpha)}\right), \quad A=1, \ldots, \mathrm{d} .
$$

\section{Killing spinors for the D3-brane solutions}

Here we provide some details of the derivation of the solution to the Killing spinor equations for the D3-brane solutions ${ }^{21}$ given in (3.6), (3.7). As in the text we choose the orthonormal frame for the metric given by

$$
\mathrm{e}^{a}=H^{1 / 6} \overline{\mathrm{e}}^{a}, \quad a=0,1,2, \quad \mathrm{e}^{3}=\frac{H^{1 / 6}}{2 P^{1 / 2}} \mathrm{~d} y, \quad \mathrm{e}^{4}=\frac{P^{1 / 2}}{H^{1 / 3}} \mathrm{~d} z,
$$

where $\overline{\mathrm{e}}^{a}$ is an orthonormal frame for the unit radius metric on $\mathrm{AdS}_{3}$. We use fivedimensional gamma matrices

$$
\Gamma_{a}=\beta_{a} \otimes \gamma_{3}, \quad \Gamma_{3}=1 \otimes \gamma_{1}, \quad \Gamma_{4}=1 \otimes \gamma_{2},
$$

where $\beta_{0}=\mathrm{i} \sigma^{2}, \beta_{1}=\sigma^{1}, \beta_{2}=\sigma^{3}$ are three-dimensional gamma matrices for $\mathrm{AdS}_{3}$ directions, while $\gamma_{1}=\sigma^{1}, \gamma_{2}=\sigma^{2}$ are two-dimensional gamma matrices for the spindle directions, with chirality matrix $\gamma_{3}=-\mathrm{i} \gamma_{1} \gamma_{2}=\sigma^{3}$. We recall that the functions appearing in the solution, given in (3.7), allows us to introduce an angle $\alpha$ defined via

$$
\sin \alpha=\frac{P^{1 / 2}}{H^{1 / 2}}, \quad \cos \alpha=\frac{y}{H^{1 / 2}} .
$$

We first consider the gaugino Killing spinor equation given in (3.4):

$$
\left[\not \partial \varphi_{i}-2 \sum_{I=1}^{3} \partial_{\varphi_{i}} X^{(I)}+\frac{\mathrm{i}}{2} \sum_{I=1}^{3} \partial_{\varphi_{i}}\left(X^{(I)}\right)^{-1} F_{\mu \nu}^{(I)} \Gamma^{\mu \nu}\right] \epsilon=0 .
$$

For the solution, and utilizing $X^{(1)} X^{(2)} X^{(3)}=1$, we can rewrite

$$
\not \varphi_{i}=-2\left(\sum_{I=1}^{3} \partial_{\varphi_{i}} X^{(I)}\right) \sin \alpha \Gamma_{3}
$$

Next, using the condition (3.8) for the flux we have

$$
\left(X^{(I)}\right)^{-1} F_{34}^{(I)}=\frac{2 X^{(I)} q_{I}}{H^{1 / 2}}=-2\left(X^{(I)} \cos \alpha-\frac{1}{H^{1 / 6}}\right)
$$

\footnotetext{
${ }^{21}$ The derivation of the Killing spinors for the case of M2-brane and M5-brane solutions runs along similar lines.
} 
and thus we can also rewrite

$$
\frac{\mathrm{i}}{2} \partial_{\varphi_{i}}\left(X^{(I)}\right)^{-1} F_{\mu \nu}^{(I)} \Gamma^{\mu \nu}=2 \mathrm{i} \partial_{\varphi_{i}} X^{(I)}\left[1-\left(X^{(I)}\right)^{-1} \frac{H^{1 / 3}}{y}\right] \cos \alpha \Gamma_{34} .
$$

Taking the sum of over $I$ we deduce that

$$
\frac{\mathrm{i}}{2} \sum_{I=1}^{3} \partial_{\varphi_{i}}\left(X^{(I)}\right)^{-1} F_{\mu \nu}^{(I)} \Gamma^{\mu \nu}=2 \mathrm{i}\left(\sum_{I=1}^{3} \partial_{\varphi_{i}} X^{(I)}\right) \cos \alpha \Gamma_{34},
$$

where we used the fact that the sum for the second term in the brackets in (C.7) vanishes, due to

$$
\sum_{I=1}^{3}\left(X^{(I)}\right)^{-1} \partial_{\varphi_{i}} X^{(I)}=\sum_{I=1}^{3} \partial_{\varphi_{i}} \log X^{(I)}=\partial_{\varphi_{i}} \log \left[X^{(1)} X^{(2)} X^{(3)}\right]=0,
$$

since $X^{(1)} X^{(2)} X^{(3)}=1$. Putting this together, for the solution we can thus rewrite (C.4) in the form

$$
2 \mathrm{i}\left(\sum_{I=1}^{3} \partial_{\varphi_{i}} X^{(I)}\right)(M+\mathrm{i}) \epsilon=0
$$

where the matrix $M$ is given by

$$
M=\cos \alpha \Gamma_{34}+\mathrm{i} \sin \alpha \Gamma_{3},
$$

introduced in (3.38), satisfying $M^{2}=-1$. We thus see that the vanishing of the gaugino variation, for both $i=1,2$, reduces to the condition that the Killing spinors $\epsilon$ satisfy

$$
M \epsilon=-\mathrm{i} \epsilon .
$$

We now turn to the gravitino equation in (3.4) given by

$$
\left[\nabla_{\mu}-\frac{\mathrm{i}}{2} \sum_{I=1}^{3} A_{\mu}^{(I)}+\frac{1}{6} \sum_{I=1}^{3} X^{(I)} \Gamma_{\mu}+\frac{\mathrm{i}}{24} \sum_{I=1}^{3}\left(X^{(I)}\right)^{-1}\left(\Gamma_{\mu}^{\nu \rho}-4 \delta_{\mu}^{\nu} \Gamma^{\rho}\right) F_{\nu \rho}^{(I)}\right] \epsilon=0 .
$$

We make an ansatz for the spinor of the form

$$
\epsilon=\vartheta \otimes \zeta,
$$

where $\vartheta$ an $\mathrm{AdS}_{3}$ Killing spinor satisfying

$$
\bar{\nabla}_{a} \vartheta=\frac{1}{2} \beta_{a} \vartheta
$$

where here $\bar{\nabla}_{a}$ is the covariant derivative on the unit radius $\mathrm{AdS}_{3}$ (as discussed in appendix B) and $\zeta$ is a two-dimensional spinor on the spindle, which we can write as

$$
\zeta=\left(\begin{array}{l}
\zeta_{+}(y, z) \\
\zeta_{-}(y, z)
\end{array}\right)
$$


The condition (C.12) that we derived above implies that $\zeta_{ \pm}$are related via

$$
\cos \frac{\alpha}{2} \zeta_{+}+\sin \frac{\alpha}{2} \zeta_{-}=0 .
$$

We first consider the components of the Killing spinor equation that are along the directions tangent to $\mathrm{AdS}_{3}$. Using the fact that

$$
\sum_{I=1}^{3} X^{(I)}=\frac{H^{\prime}}{H^{2 / 3}}
$$

as well as (C.15) we obtain

$$
\nabla_{a} \epsilon=\left[-\frac{\mathrm{i}}{2 H^{1 / 6}} \Gamma_{34}-\frac{1}{6}\left(\sum_{I=1}^{3} X^{(I)}\right) \sin \alpha \Gamma_{3}\right] \Gamma_{a} \epsilon
$$

Then using (C.6) we deduce that for these components we must have

$$
-\frac{\mathrm{i}}{6}\left(\sum_{I=1}^{3} X^{(I)}\right) \Gamma_{a}(M+\mathrm{i}) \epsilon=0
$$

which vanishes by virtue of the projection (C.12).

We next consider the components tangent to the spindle $\Sigma$. The spin connection associated to $\widetilde{\mathrm{d} s^{2}} \Sigma$ is given by $\widetilde{\omega}^{12}=\widetilde{\omega}_{4}^{12} \mathrm{e}^{4}$, where

$$
\widetilde{\omega}_{4}^{12}=\frac{2 P H^{\prime}-3 H P^{\prime}}{3 H^{7 / 6} P^{1 / 2}} .
$$

We now consider the components of the gravitino variation along the $\mathrm{e}^{4}$ direction. We have

$$
\nabla_{4} \epsilon=\frac{1}{H^{1 / 6} \sin \alpha} \partial_{z} \epsilon+\frac{1}{2} \widetilde{\omega}_{4}^{12} \Gamma_{34} \epsilon .
$$

The gauge fields can be rewritten as

$$
A_{4}^{(I)}=\frac{\cos \alpha}{\sin \alpha} X^{(I)},
$$

and using (C.6) we obtain the condition

$$
\left[\frac{1}{H^{1 / 6} \sin \alpha} \partial_{z}+\frac{1}{2} \widetilde{\omega}_{4}^{12} \Gamma_{34}-\left(\sum_{I=1}^{3} X^{(I)}\right)\left(\frac{\mathrm{i}}{2} \frac{\cos \alpha}{\sin \alpha}-\frac{1}{6} \Gamma_{4}+\frac{\mathrm{i}}{3} \cos \alpha \Gamma_{3}\right)+\frac{\mathrm{i}}{H^{1 / 6}} \Gamma_{3}\right] \epsilon=0 .
$$

This requires some massaging. First, we use (C.11) to rewrite

$$
\frac{\mathrm{i}}{2} \frac{\cos \alpha}{\sin \alpha}=-\frac{1}{2}\left(\frac{\cos ^{2} \alpha}{\sin \alpha} \Gamma_{34}+\mathrm{i} \cos \alpha \Gamma_{3}\right)+\frac{1}{2} \frac{\cos \alpha}{\sin \alpha}(M+\mathrm{i}) .
$$

Combining this with

$$
\frac{1}{2} \widetilde{\omega}_{4}^{12}+\frac{1}{2} \frac{\cos ^{2} \alpha}{\sin \alpha}\left(\sum_{I=1}^{3} X^{(I)}\right)=\frac{\cos \alpha}{H^{1 / 6} \sin \alpha}-\frac{1}{6} \sin \alpha\left(\sum_{I=1}^{3} X^{(I)}\right),
$$


we obtain

$$
\frac{1}{H^{1 / 6} \sin \alpha}\left[\partial_{z}+M-H^{1 / 6}\left(\sum_{I=1}^{3} X^{(I)}\right)\left(\frac{\mathrm{i}}{6} \sin \alpha \Gamma_{4}+\frac{1}{2} \cos \alpha\right)(M+\mathrm{i})\right] \epsilon=0 .
$$

Using (C.12) this finally reduces to $\left(\partial_{z}-\mathrm{i}\right) \epsilon=0$ so that the spinor $\zeta$ on the spindle must satisfy

$$
\partial_{z} \zeta=\mathrm{i} \zeta
$$

This can be solved by setting

$$
\zeta=\mathrm{e}^{\mathrm{i} z \bar{\zeta}}=\left(\begin{array}{l}
\bar{\zeta}_{+}(y) \\
\bar{\zeta}_{-}(y)
\end{array}\right)
$$

with $\bar{\zeta}_{ \pm}$subject to the same constraint (C.17) as $\zeta_{ \pm}$.

Finally, let us turn to the components along $\mathrm{e}^{3}$. Here we again use (C.6) to obtain the condition

$$
\left[2 H^{1 / 3} \sin \alpha \partial_{y}+\frac{1}{6}\left(\sum_{I=1}^{3} X^{(I)}\right)\left(\Gamma_{3}+2 \mathrm{i} \cos \alpha \Gamma_{4}\right)-\frac{\mathrm{i}}{H^{1 / 6}} \Gamma_{4}\right] \epsilon=0 .
$$

Writing $\epsilon=\mathrm{e}^{\mathrm{i} z} \vartheta \otimes \bar{\zeta}$, and using the explicit choice of gamma matrices given in and below (C.2), as well as (C.18) we obtain the ODE

$$
\partial_{y} \bar{\zeta}=\left[-\frac{H^{\prime}}{12 H^{1 / 2} P^{1 / 2}}\left(\sigma^{1}+2 \mathrm{i} \sigma^{2}\right)+\frac{\mathrm{i}}{2 P^{1 / 2}} \sigma^{2}\right] \bar{\zeta} .
$$

Now, using the relation between $\bar{\zeta}_{ \pm}$given in (C.17), we find that the first component of this ODE is

$$
\partial_{y} \bar{\zeta}_{+}=\frac{-6 H+H^{\prime}\left(2 y+H^{1 / 2}\right)}{12 H P^{1 / 2}} \cot \frac{\alpha}{2} \bar{\zeta}_{+},
$$

which is solved by

$$
\bar{\zeta}_{+}=H^{1 / 12} \sin \frac{\alpha}{2}, \quad \Rightarrow \quad \bar{\zeta}_{-}=-H^{1 / 12} \cos \frac{\alpha}{2} .
$$

One can check that this solution also solves the other component of the ODE (C.31). We have thus derived the Killing spinor solution given in (3.36).

Open Access. This article is distributed under the terms of the Creative Commons Attribution License (CC-BY 4.0), which permits any use, distribution and reproduction in any medium, provided the original author(s) and source are credited.

\section{References}

[1] P. Ferrero, J.P. Gauntlett, J.M. Pérez Ipiña, D. Martelli and J. Sparks, D3-branes wrapped on a spindle, Phys. Rev. Lett. 126 (2021) 111601 [arXiv:2011.10579] [InSPIRE].

[2] F. Faedo and D. Martelli, D4-branes wrapped on a spindle, arXiv:2111.13660 [INSPIRE].

[3] P. Ferrero, J.P. Gauntlett, J.M.P. Ipiña, D. Martelli and J. Sparks, Accelerating black holes and spinning spindles, Phys. Rev. D 104 (2021) 046007 [arXiv: 2012.08530] [INSPIRE]. 
[4] S.M. Hosseini, K. Hristov and A. Zaffaroni, Rotating multi-charge spindles and their microstates, JHEP 07 (2021) 182 [arXiv:2104.11249] [INSPIRE].

[5] A. Boido, J.M.P. Ipiña and J. Sparks, Twisted D3-brane and M5-brane compactifications from multi-charge spindles, JHEP 07 (2021) 222 [arXiv:2104.13287] [INSPIRE].

[6] P. Ferrero, J.P. Gauntlett, D. Martelli and J. Sparks, M5-branes wrapped on a spindle, JHEP 11 (2021) 002 [arXiv:2105.13344] [INSPIRE].

[7] P. Ferrero, M. Inglese, D. Martelli and J. Sparks, Multi-charge accelerating black holes and spinning spindles, arXiv:2109.14625 [INSPIRE].

[8] C. Couzens, K. Stemerdink and D. van de Heisteeg, M2-branes on discs and multi-charged spindles, arXiv:2110.00571 [INSPIRE].

[9] J.P. Gauntlett, O.A.P. Mac Conamhna, T. Mateos and D. Waldram, Supersymmetric $A d S_{3}$ solutions of type IIB supergravity, Phys. Rev. Lett. 97 (2006) 171601 [hep-th/0606221] [INSPIRE].

[10] J.P. Gauntlett, N. Kim and D. Waldram, Supersymmetric $A d S_{3}, A d S_{2}$ and bubble solutions, JHEP 04 (2007) 005 [hep-th/0612253] [INSPIRE].

[11] J.M. Maldacena and C. Núñez, Supergravity description of field theories on curved manifolds and a no go theorem, Int. J. Mod. Phys. A 16 (2001) 822 [hep-th/0007018] [inSPIRE].

[12] C. Closset, T.T. Dumitrescu, G. Festuccia and Z. Komargodski, Supersymmetric field theories on three-manifolds, JHEP 05 (2013) 017 [arXiv:1212.3388] [INSPIRE].

[13] I. Bah, F. Bonetti, R. Minasian and E. Nardoni, Holographic duals of Argyres-Douglas theories, Phys. Rev. Lett. 127 (2021) 211601 [arXiv:2105.11567] [InSPIRE].

[14] I. Bah, F. Bonetti, R. Minasian and E. Nardoni, M5-brane sources, holography, and Argyres-Douglas theories, JHEP 11 (2021) 140 [arXiv:2106.01322] [INSPIRE].

[15] C. Couzens, N.T. Macpherson and A. Passias, $N=(2,2)$ Ad $S_{3}$ from D3-branes wrapped on Riemann surfaces, arXiv:2107.13562 [INSPIRE].

[16] M. Suh, D3-branes and M5-branes wrapped on a topological disc, arXiv:2108.01105 [INSPIRE].

[17] J.P. Gauntlett, D. Martelli, J. Sparks and D. Waldram, Supersymmetric AdS $S_{5}$ solutions of M-theory, Class. Quant. Grav. 21 (2004) 4335 [hep-th/0402153] [INSPIRE].

[18] M. Gabella, D. Martelli, A. Passias and J. Sparks, $N=2$ supersymmetric AdS $S_{4}$ solutions of M-theory, Commun. Math. Phys. 325 (2014) 487 [arXiv:1207.3082] [INSPIRE].

[19] A. Passias and D. Prins, On AdS 3 solutions of type IIB, JHEP 05 (2020) 048 [arXiv: 1910.06326] [INSPIRE].

[20] T.T. Dumitrescu, G. Festuccia and N. Seiberg, Exploring curved superspace, JHEP 08 (2012) 141 [arXiv:1205.1115] [INSPIRE].

[21] F. Benini and N. Bobev, Two-dimensional SCFTs from wrapped branes and c-extremization, JHEP 06 (2013) 005 [arXiv: 1302.4451] [inSPIRE].

[22] K. Behrndt, A.H. Chamseddine and W.A. Sabra, BPS black holes in $N=2$ five-dimensional AdS supergravity, Phys. Lett. B 442 (1998) 97 [hep-th/9807187] [INSPIRE].

[23] M. Cvetič et al., Embedding AdS black holes in ten-dimensions and eleven-dimensions, Nucl. Phys. B 558 (1999) 96 [hep-th/9903214] [INSPIRE]. 
[24] H. Lin, O. Lunin and J.M. Maldacena, Bubbling AdS space and 1/2 BPS geometries, JHEP 10 (2004) 025 [hep-th/0409174] [InSPIRE].

[25] J.P. Gauntlett and O. Varela, $D=5 \mathrm{SU}(2) \times \mathrm{U}(1)$ gauged supergravity from $D=11$ supergravity, JHEP 02 (2008) 083 [arXiv:0712.3560] [INSPIRE].

[26] D. Gaiotto and J. Maldacena, The gravity duals of $N=2$ superconformal field theories, JHEP 10 (2012) 189 [arXiv:0904.4466] [InSPIRE].

[27] A. Buchel and J.T. Liu, Gauged supergravity from type IIB string theory on $Y^{p, q}$ manifolds, Nucl. Phys. B 771 (2007) 93 [hep-th/0608002] [INSPIRE].

[28] H.K. Kunduri and J. Lucietti, Near-horizon geometries of supersymmetric AdS $S_{5}$ black holes, JHEP 12 (2007) 015 [arXiv:0708.3695] [INSPIRE].

[29] A. Donos, J.P. Gauntlett and C. Pantelidou, Magnetic and electric AdS solutions in stringand M-theory, Class. Quant. Grav. 29 (2012) 194006 [arXiv:1112.4195] [INSPIRE].

[30] H. Lü and J.F. Vázquez-Poritz, C-metrics in gauged STU supergravity and beyond, JHEP 12 (2014) 057 [arXiv: 1408.6531] [INSPIRE].

[31] J.T. Liu and R. Minasian, Black holes and membranes in AdS $S_{7}$, Phys. Lett. B 457 (1999) 39 [hep-th/9903269] [INSPIRE].

[32] H. Nastase, D. Vaman and P. van Nieuwenhuizen, Consistent nonlinear $K K$ reduction of $11 D$ supergravity on $A d S_{7} \times S^{4}$ and selfduality in odd dimensions, Phys. Lett. B 469 (1999) 96 [hep-th/9905075] [INSPIRE].

[33] J.P. Gauntlett and N. Kim, Geometries with Killing spinors and supersymmetric AdS solutions, Commun. Math. Phys. 284 (2008) 897 [arXiv:0710.2590] [INSPIRE].

[34] C. Couzens, J.P. Gauntlett, D. Martelli and J. Sparks, A geometric dual of c-extremization, JHEP 01 (2019) 212 [arXiv:1810.11026] [INSPIRE].

[35] J.P. Gauntlett, D. Martelli and J. Sparks, Toric geometry and the dual of c-extremization, JHEP 01 (2019) 204 [arXiv:1812.05597] [INSPIRE].

[36] S.M. Hosseini and A. Zaffaroni, Geometry of $\mathcal{I}$-extremization and black holes microstates, JHEP 07 (2019) 174 [arXiv:1904.04269] [INSPIRE].

[37] J.P. Gauntlett, D. Martelli and J. Sparks, Toric geometry and the dual of $\mathcal{I}$-extremization, JHEP 06 (2019) 140 [arXiv:1904.04282] [INSPIRE].

[38] H. Kim and N. Kim, Black holes with baryonic charge and $\mathcal{I}$-extremization, JHEP 11 (2019) 050 [arXiv: 1904.05344$]$ [INSPIRE].

[39] J.P. Gauntlett, D. Martelli and J. Sparks, Fibred GK geometry and supersymmetric AdS solutions, JHEP 11 (2019) 176 [arXiv:1910.08078] [INSPIRE].

[40] S.L. Cacciatori and D. Klemm, Supersymmetric AdS 4 black holes and attractors, JHEP 01 (2010) 085 [arXiv:0911.4926] [INSPIRE].

[41] I. Bah, C. Beem, N. Bobev and B. Wecht, Four-dimensional SCFTs from M5-branes, JHEP 06 (2012) 005 [arXiv: 1203.0303] [INSPIRE].

[42] H. Lü, C.N. Pope and P.K. Townsend, Domain walls from anti-de Sitter space-time, Phys. Lett. B 391 (1997) 39 [hep-th/9607164] [INSPIRE].

[43] H. Lü, C.N. Pope and J. Rahmfeld, A construction of Killing spinors on $S^{n}$, J. Math. Phys. 40 (1999) 4518 [hep-th/9805151] [INSPIRE]. 